\title{
Pd-Catalyzed Silicon Hydride Reductions of Aromatic and Aliphatic Nitro Groups
}

Ronald J. Rahaim, Jr. and Robert E. Maleczka, Jr.*

Department of Chemistry, Michigan State University, East Lansing, MI 48824

Maleczka@cem.msu.edu

Supporting Information

Index

Materials and Methods

General Procedure for the Reduction of Nitro Arenes to Amines

General Procedure for the Reduction of Aliphatic Nitro Compounds

S2-S3

Experimental Details and Spectroscopic Data

S3-S11

Preparation of 6-Bromohexyl 4-Nitrobenzoate S10-S11

${ }^{1} \mathrm{H}$ and ${ }^{13} \mathrm{C}$ NMR Spectra for Corresponding Products

S12-S89 
Materials and Methods: All reactions were carried out in oven dried glassware under an atmosphere of nitrogen, with magnetic stirring, and monitored by thin-layer chromatography with $0.25-\mathrm{mm}$ precoated silica gel plates, unless otherwise noted. Tetrahydrofuran was freshly distilled from sodium/benzophenone under nitrogen. Palladium (II) acetate, anhydrous A.C.S grade potassium fluoride, polymethylhydrosiloxane (PMHS), and triethylsilane were purchased and used without purification. 4-Acetoxynitrobenzene, ${ }^{1}$ tert-butyldimethyl-(4-nitrophenoxy)silane, ${ }^{2}$ 6-bromohexyl 4-nitrobenzoate, 2-nitrothiophene, ${ }^{3}$ 1-nitrodecane, ${ }^{4}$ (2-nitroethyl)benzene, ${ }^{4}$ 1,3diaetoxy-2-acetoxymethyl-2-nitropropane, ${ }^{5}$ 4-nitro-1-phenyl-3-pentanol, ${ }^{6}$ and syn-2-(2-nitrophenyl-ethyl)cyclohexanone ${ }^{7}$ were prepared following literature procedures. The remaining nitro compounds were purchased and used without purification, except for 4-nitroanisole which was recrystallized from hexanes before use. Flash chromatography was performed with silica gel 60 $\AA$ (230-400 mesh). Yields refer to chromatographically and spectroscopically pure compounds unless other wise stated. ${ }^{1} \mathrm{H}$ NMR and ${ }^{13} \mathrm{C}$ NMR spectra were recorded on a $300 \mathrm{MHz}$ spectrometer $\left(300 \mathrm{MHz}\right.$ for ${ }^{1} \mathrm{H}$, respectively, and $75 \mathrm{MHz}$ for ${ }^{13} \mathrm{C}$, respectively), with chemical shifts reported relative to the residue peaks of solvent chloroform $\left(\delta 7.24\right.$ for ${ }^{1} \mathrm{H}$ and 77.0 for $\left.{ }^{13} \mathrm{C}\right)$ or dimethyl sulfoxide ( $\delta 2.50$ for ${ }^{1} \mathrm{H}$ and 39.5 for ${ }^{13} \mathrm{C}$ ). Melting points were measured on a capillary melting point apparatus and are uncorrected.

General Procedure for the Reduction of Nitro Arenes to Amines: A round bottom flask was charged with palladium acetate $(0.05 \mathrm{mmol}, 11 \mathrm{mg})$, the nitroarene $(1 \mathrm{mmol})$, and freshly distilled dry THF $(5 \mathrm{~mL})$. The flask was sealed and purged with nitrogen. While purging the flask with nitrogen a solution of aqueous $\mathrm{KF}$ was added via syringe $(2 \mathrm{mmol} \mathrm{KF}, 116 \mathrm{mg}$; in $2 \mathrm{~mL}$ of degassed water). The nitrogen inlet was replaced with a balloon of nitrogen. PMHS (4 mmol, $0.24 \mathrm{~mL} ; 1 \mathrm{mmol}$ of hydride is $0.06 \mathrm{~mL}$ ) was slowly added dropwise via syringe (Caution: Rapid addition PMHS can result in uncontrollable gas evolution!) The reaction was stirred for 30 min or until complete as judged by TLC. At that time, the reaction flask was opened to the air, diluted with $5-10 \mathrm{~mL}$ of diethyl ether, and stirred for 5 minutes. The layers were separated and the aqueous layer was back extracted with diethyl ether. The combined organics were filtered through a plug of Celite (top layer) and neutral alumina (bottom layer) in a $1 \mathrm{~cm}$ diameter column by flushing with EtOAc. The filtrate was concentrated and subjected to flash chromatography using gradients of hexanes/EtOAc and/or EtOAc/MeOH. (Note: In the case of carboxylic acid 27, thiophene 32, and benzimidazole 33, reaction mixture extracts were filtered though a plug of only Celite (No neutral alumina) before flash chromatography.)

General Procedure For the Reduction of Aliphatic Nitro Compounds: A round bottom flask was charged with palladium acetate $(0.05 \mathrm{mmol}, 11 \mathrm{mg})$, the aliphatic nitro compound $(1 \mathrm{mmol})$, and freshly distilled dry THF $(5 \mathrm{~mL})$. The flask was sealed and purged with nitrogen. While purging the flask with nitrogen $2 \mathrm{~mL}$ of degassed $\mathrm{H}_{2} \mathrm{O}$ was added via syringe. ${ }^{8}$ The nitrogen inlet was replaced with a balloon of nitrogen. Triethylsilane $(6 \mathrm{mmol}, 0.96 \mathrm{~mL})$ was slowly added dropwise via syringe. The reaction was stirred for 2 hours or until complete as judged by TLC. At

(1) Hanzlik, R. P. Org. Synth. CV 6, 560.

(2) Corey, E. J.; Venkateswarlu, A J. Am. Chem. Soc. 1972, 94, $6190 z 6191$.

(3) Babasin, V. S. Org. Syn., CV 2, 466.

(4) Kornblum, N.; Powers, J. W. J. Org. Chem. 1957, 22, 455456.

(5) Guanti, G.; Banfi, L.; Nariasano, E. J. Org. Chem. 1992, 57, 1540-1554.

(6) Sasai, H.; Watanabe, S.; Suzuki, T.; Shibasaki, M. Org. Synth. CV 10, 571.

(7) List, B.; Projarliev, P.; Martin, H. J. Org. Lett. 2001, 3, 2423-2425.

(8) The procedure published in the orginal supporting information mistakenly called for the addition of aqueous KF at this stage. This corrected version was submitted to the Editor on March 14, 2006. 
that time, the reaction flask was opened to the air, diluted with $5-10 \mathrm{~mL}$ of diethyl ether, and stirred for 5 minutes. The layers were separated and the aqueous layer was back extracted with diethyl ether. The combined organics were concentrated and subjected to flash chromatography using gradients of hexanes/EtOAc and/or EtOAc/MeOH.

\section{Experimental Details and Spectroscopic Data:}

2-Aminotoluene (1): Subjection of 2-nitrotoluene $(1 \mathrm{mmol}, 0.117 \mathrm{~mL})$ to the general procedure for reducing nitro arenes afforded $0.107 \mathrm{~g}(100 \%)$ of 2-aminotoluene (1) as a yellow oil (silica gel FC, hexanes/EtOAc: 80/20 then 50/50). ${ }^{1} \mathrm{H}$ NMR (300 MHz, $\left.\mathrm{CDCl}_{3}\right): \delta 7.06(\mathrm{~m}, 2 \mathrm{H}), 6.79-6.62$ $(\mathrm{m}, 2 \mathrm{H}), 3.60$ (bs, $2 \mathrm{H}), 2.18$ (s, 3H); ${ }^{13} \mathrm{C}$ NMR $\left(75 \mathrm{MHz}, \mathrm{CDCl}_{3}\right): \delta 144.4,130.3,126.8,122.2$, $118.5,114.8,17.2$. Physical and spectral data were consistent with commercially available material.

4-Aminotoluene (2): Subjection of 4-nitrotoluene $(1 \mathrm{mmol}, 0.1371 \mathrm{~g})$ to the general procedure for reducing nitro arenes afforded $0.101 \mathrm{~g}(94 \%)$ of 4-aminotoluene (2) as a yellow-orange solid (silica gel FC, hexanes/EtOAc: 80/20 then 50/50); $\mathrm{mp}=40-44^{\circ} \mathrm{C} .{ }^{1} \mathrm{H} \mathrm{NMR}\left(300 \mathrm{MHz}, \mathrm{CDCl}_{3}\right): \delta$ $6.97(\mathrm{~d}, J=8.24 \mathrm{~Hz}, 2 \mathrm{H}), 6.61(\mathrm{~d}, J=8.24 \mathrm{~Hz}, 2 \mathrm{H}), 3.52(\mathrm{bs}, 2 \mathrm{H}), 2.24(\mathrm{~s}, 3 \mathrm{H}) ;{ }^{13} \mathrm{C}$ NMR $(75$ $\left.\mathrm{MHz}, \mathrm{CDCl}_{3}\right): \delta 143.7,129.6,127.6,115.2,20.3$. Physical and spectral data were consistent with commercially available material.

2-Aminoaniline (3): Subjection of 2-nitroaniline $(1 \mathrm{mmol}, 0.138 \mathrm{~g})$ to the general procedure for reducing nitro arenes (full consumption of starting material as judged by TLC) followed by the addition of acetic anhydride $(4 \mathrm{mmol}, 0.376 \mathrm{~mL})$ to the reaction mixture with an additional 30 minutes of stirring, afforded $0.1902 \mathrm{~g}(99 \%)$ of $N, N^{\prime}-(1,2-$ phenylene)diacetamide as a white solid (silica gel FC, EtOAc/MeOH: 100/0 then 50/50); $\mathrm{mp}=183-185^{\circ} \mathrm{C}$. ${ }^{1} \mathrm{H}$ NMR $(300 \mathrm{MHz}$, DMSO$\left.d_{6}\right): \delta 9.31(\mathrm{~s}, 2 \mathrm{H}), 7.53(\mathrm{~m}, 2 \mathrm{H}), 7.10(\mathrm{~m}, 2 \mathrm{H}), 2.05(\mathrm{~s}, 6 \mathrm{H}) ;{ }^{13} \mathrm{C}$ NMR $\left(75 \mathrm{MHz}\right.$, DMSO- $\left.d_{6}\right): \delta$ $168.6,130.4,124.7,124.5,23.6$. Physical and spectral data were consistent with those previously reported. $^{9}$

1,3-Benzenediamine (4): Subjection of 3-nitroaniline $(1 \mathrm{mmol}, 0.138 \mathrm{~g})$ to the general procedure for reducing nitro arenes afforded $0.108 \mathrm{~g}(99 \%)$ of 1,3-benzenediamine (4) as a green oil (silica gel FC, hexanes/EtOAc: 50/50 then 0/100). ${ }^{1} \mathrm{H}$ NMR $\left(300 \mathrm{MHz}, \mathrm{CDCl}_{3}+\mathrm{DMSO}-d_{6}\right): \delta 6.56(\mathrm{t}, \mathrm{J}=$ $7.69 \mathrm{~Hz}, 1 \mathrm{H}), 5.74(\mathrm{dd}, J=8.24 \mathrm{~Hz}, 2 \mathrm{H}), 5.69(\mathrm{t}, J=2.19,1 \mathrm{H}), 3.61(\mathrm{~s}, 4 \mathrm{H}) ;{ }^{13} \mathrm{C} \mathrm{NMR}(75 \mathrm{MHz}$, $\left.\mathrm{CDCl}_{3}+\mathrm{DMSO}_{-} d_{6}\right): \delta 147.1,129.0,104.5,100.8$. Physical and spectral data were consistent with commercially available material.

1,4-Benzenediamine (5): Subjection of 4-nitroaniline $(1 \mathrm{mmol}, 0.138 \mathrm{~g})$ to the general procedure for reducing nitro arenes afforded $0.1033 \mathrm{~g}(95 \%)$ of 1,4-benzenediamine (5) as a purple solid (silica gel FC, hexanes/EtOAc: 50/50 then 0/100); $\mathrm{mp}=141^{\circ} \mathrm{C} .{ }^{1} \mathrm{H}$ NMR $\left(300 \mathrm{MHz}, \mathrm{CDCl}_{3}+\right.$ DMSO- $\left.d_{6}\right): \delta 6.32$ (s, 4H), 3.28 (bs, 4H); ${ }^{13} \mathrm{C}$ NMR (75 MHz, CDCl 3 + DMSO- $\left.d_{6}\right): \delta$ 138.1, 116.0. Physical and spectral data were consistent with commercially available material.

2-Aminophenol (6): Subjection of 2-nitrophenol (1 mmol, $0.139 \mathrm{~g})$ to the general procedure for reducing nitro arenes afforded $0.1025 \mathrm{~g}(94 \%)$ of 2-aminophenol (6) and $0.0052 \mathrm{~g} \mathrm{(4 \% )}$ of the oxidized product as a red-yellow solid. For 2-aminophenol (6): ${ }^{1} \mathrm{H}$ NMR $\left(300 \mathrm{MHz}, \mathrm{CDCl}_{3}+\right.$ DMSO- $d_{6}$ ): $\delta 8.38$ (bs, $\left.1 \mathrm{H}\right), 6.56-6.25(\mathrm{~m}, 4 \mathrm{H}), 3.62$ (bs, $\left.2 \mathrm{H}\right) ;{ }^{13} \mathrm{C} \mathrm{NMR}\left(75 \mathrm{MHz}, \mathrm{CDCl}_{3}+\mathrm{DMSO}\right.$ $\left.d_{6}\right): \delta 144.0,134.8,119.4,117.7,114.9,114.4$. Analytically pure material was obtained by following the general procedure for reducing nitro arenes with the following modification. After

(9) Lovel, I.; Golomba, L.; Popelis, J.; Gaukhman, A.; Lukevics, E. Appl. Organomet. Chem. 2001, 67-74. 
complete consumption of the starting material (as judged by TLC) acetic anhydride ( $2 \mathrm{mmol}$, $0.188 \mathrm{~mL}$ ) was added to the reaction mixture followed by an additional 30 minutes of stirring to afford after the general workup $0.1481 \mathrm{~g}(98 \%)$ of 2-acetamidophenol as a white solid (silica gel FC, hexanes/EtOAc: 80/20, 50/50 then 0/100); mp $=205{ }^{\circ} \mathrm{C}$. ${ }^{1} \mathrm{H}$ NMR $\left(300 \mathrm{MHz}\right.$, DMSO- $\left.d_{6}\right): \delta$ $9.70(\mathrm{~s}, 1 \mathrm{H}), 9.29(\mathrm{~s}, 1 \mathrm{H}), 7.64(\mathrm{~d}, J=\mathrm{Hz}, 2 \mathrm{H}), 6.90-6.70(\mathrm{~m}, 3 \mathrm{H}), 2.05(\mathrm{~s}, 3 \mathrm{H}) ;{ }^{13} \mathrm{C}$ NMR $(75$ $\left.\mathrm{MHz}, \mathrm{DMSO}-d_{6}\right): \delta 169.1,147.9,126.4,124.7,122.4,119.0,115.9,23.6$. Physical and spectral data were consistent with commercially available material.

3-Aminophenol (7): Subjection of 3-nitrophenol ( $1 \mathrm{mmol}, 0.139 \mathrm{~g})$ to the general procedure for reducing nitro arenes afforded $0.1031 \mathrm{~g}(94 \%)$ of 3-aminophenol (7) as a tan solid (silica gel FC, hexanes/EtOAc: 50/50); $\mathrm{mp}=119-120{ }^{\circ} \mathrm{C}$. ${ }^{1} \mathrm{H}$ NMR $\left(300 \mathrm{MHz}, \mathrm{CDCl}_{3}+\mathrm{DMSO}_{6}\right): \delta 8.31$ (bs, $1 \mathrm{H}), 6.60(\mathrm{t}, J=8.24 \mathrm{~Hz}, 1 \mathrm{H}), 5.84(\mathrm{dt}, J=9.89 \mathrm{~Hz}, 3 \mathrm{H}), 3.62(\mathrm{bs}, 2 \mathrm{H}) ;{ }^{13} \mathrm{C} \mathrm{NMR}\left(75 \mathrm{MHz}, \mathrm{CDCl}_{3}\right.$ + DMSO- $\left.d_{6}\right): \delta 157.4,147.6,129.2,105.7,104.5,101.4$. Physical and spectral data were consistent with commercially available material.

4-Aminophenol (8): Subjection of 4-nitrophenol $(1 \mathrm{mmol}, 0.139 \mathrm{~g})$ to the general procedure for reducing nitro arenes afforded $0.0994 \mathrm{~g}(91 \%)$ of 4 -aminophenol (8) and $0.008 \mathrm{~g}(7 \%)$ of the oxidized product as a tan solid. For 4-aminophenol (8): ${ }^{1} \mathrm{H}$ NMR $\left(300 \mathrm{MHz}, \mathrm{CDCl}_{3}+\mathrm{DMSO}_{6} d_{6}\right)$ : $\delta 6.28(\mathrm{~d}, J=8.24 \mathrm{~Hz}, 2 \mathrm{H}), 6.19(\mathrm{~d}, J=8.24 \mathrm{~Hz}, 2 \mathrm{H}), 3.00(\mathrm{bs}, 2 \mathrm{H}) ;{ }^{13} \mathrm{C} \mathrm{NMR}\left(75 \mathrm{MHz}, \mathrm{CDCl}_{3}+\right.$ DMSO- $\left.d_{6}\right): \delta 148.85,138.41,115.58,115.22$. Analytically pure material was obtained by following the general procedure for reducing nitro arenes with the following modification. After complete consumption of the starting material (as judged by TLC) acetic anhydride ( $2 \mathrm{mmol}$, $0.188 \mathrm{~mL}$ ) was added to the reaction mixture followed by an additional 30 minutes of stirring to afford after the general workup $0.1496 \mathrm{~g}(99 \%)$ of acetaminophen as an off white solid (silica gel FC, hexanes/EtOAc: 80/20, 50/50 then 0/100); $\mathrm{mp}=166-168{ }^{\circ} \mathrm{C} .{ }^{1} \mathrm{H}$ NMR $\left(300 \mathrm{MHz}\right.$, DMSO- $\left.d_{6}\right)$ : $\delta 9.61(\mathrm{~s}, 1 \mathrm{H}), 9.11(\mathrm{~s}, 1 \mathrm{H}), 7.31(\mathrm{~d}, J=\mathrm{Hz}, 2 \mathrm{H}), 6.64(\mathrm{~d}, J=\mathrm{Hz}, 2 \mathrm{H}), 1.94(\mathrm{~s}, 3 \mathrm{H}) ;{ }^{13} \mathrm{C}$ NMR $(75$ $\left.\mathrm{MHz}, \mathrm{DMSO}-d_{6}\right): \delta 167.7,153.2,131.1,121.0,115.1,23.8$. Physical and spectral data were consistent with commercially available material.

2-Fluoroaniline (9): Subjection of 1-fluoro-2-nitrobenzene (1 mmol, $0.105 \mathrm{~mL})$ to the general procedure for reducing nitro arenes afforded $0.1087 \mathrm{~g} \mathrm{(97 \% )} \mathrm{of} \mathrm{2-fluoroaniline} \mathrm{(9)} \mathrm{as} \mathrm{a} \mathrm{light} \mathrm{yellow}$ oil (silica gel FC, hexanes/EtOAc: 80/20). ${ }^{1} \mathrm{H}$ NMR (300 MHz, $\left.\mathrm{CDCl}_{3}\right): \delta 7.00-6.86(\mathrm{~m}, 2 \mathrm{H})$, 6.78-6.18 (m, 2H), 3.67 (bs, 2H); ${ }^{13} \mathrm{C}$ NMR $\left(75 \mathrm{MHz}, \mathrm{CDCl}_{3}\right): \delta 153.2,150.0,134.5,124.4,124.3$, $118.5,118.4,116.8,115.2$. Physical and spectral data were consistent with commercially available material.

3-Fluoroaniline (10): Subjection of 1-fluoro-4-nitrobenzene (1 mmol, $0.106 \mathrm{~mL})$ to the general procedure for reducing nitro arenes afforded $0.1069 \mathrm{~g}(96 \%)$ of 3-fluoroaniline (10) as a light yellow oil (silica gel FC, hexanes/EtOAc: 80/20, then 50/50); ${ }^{1} \mathrm{H} \mathrm{NMR}\left(300 \mathrm{MHz}, \mathrm{CDCl}_{3}\right): \delta 7.03$ (q, $J=8.24 \mathrm{~Hz}, 1 \mathrm{H}), 6.45-6.28(\mathrm{~m}, 3 \mathrm{H}), 3.74(\mathrm{bs}, 2 \mathrm{H}) ;{ }^{13} \mathrm{C}$ NMR $\left(75 \mathrm{MHz}, \mathrm{CDCl}_{3}\right): \delta 165.3,162.1$, $148.3,148.1,130.3,130.2,110.5,104.9,104.6,101.9,101.6$. Physical and spectral data were consistent with commercially available material.

4-Fluoroaniline (11): Subjection of 1-fluoro-4-nitrobenzene $(1 \mathrm{mmol}, 0.106 \mathrm{~mL})$ to the general procedure for reducing nitro arenes afforded $0.1051 \mathrm{~g}(95 \%)$ of 4 -fluoroaniline (11) as a light yellow oil (silica gel FC, hexanes/EtOAc: 80/20 then 50/50). ${ }^{1} \mathrm{H} \mathrm{NMR}\left(300 \mathrm{MHz}, \mathrm{CDCl}_{3}\right): \delta 6.83$ $(\mathrm{m}, 2 \mathrm{H}), 6.60(\mathrm{~m}, 2 \mathrm{H}), 3.51$ (bs, $2 \mathrm{H}) ;{ }^{13} \mathrm{C}$ NMR $\left(75 \mathrm{MHz}, \mathrm{CDCl}_{3}\right): \delta 157.9,154.7,142.3,116.0$, $115.9,115.7,115.4$. Physical and spectral data were consistent with commercially available material. 
Methyl 2-aminobenzoate (12): Subjection of methyl 2-nitrobenzoate $(1 \mathrm{mmol}, 0181 \mathrm{~g})$ to the general procedure for reducing nitro arenes afforded $0.1511 \mathrm{~g}(100 \%)$ of methyl 2-aminobenzoate (12) as a bright yellow oil (silica gel FC, hexanes/EtOAc: $80 / 20)$. ${ }^{1} \mathrm{H}$ NMR $\left(300 \mathrm{MHz}, \mathrm{CDCl}_{3}\right): \delta$ 7.83 (dd, $J=1.64$ and $8.24 \mathrm{~Hz}, 1 \mathrm{H}$ ), $7.23(\mathrm{dt}, J=1.64$ and $7.14 \mathrm{~Hz}, 1 \mathrm{H}), 6.61(\mathrm{~m}, 2 \mathrm{H}), 5.69$ (bs, 2H), $3.83(\mathrm{~s}, 3 \mathrm{H}) ;{ }^{13} \mathrm{C}$ NMR $\left(75 \mathrm{MHz}, \mathrm{CDCl}_{3}\right): \delta 168.5,150.3,133.9,131.2,116.5,116.1,110.5$, 51.3. Physical and spectral data were consistent with commercially available material.

Methyl 3-aminobenzoate (13): Subjection of methyl 3-nitrobenzoate $(1 \mathrm{mmol}, 0.181 \mathrm{~g})$ to the general procedure for reducing nitro arenes afforded $0.1511 \mathrm{~g}(100 \%)$ of methyl 3-aminobenzoate (13) as a light yellow oil (silica gel FC, hexanes/EtOAc: 80/20 then 50/50). ${ }^{1} \mathrm{H}$ NMR $(300 \mathrm{MHz}$, $\left.\mathrm{CDCl}_{3}\right): \delta 7.35(\mathrm{~d}, J=7.69 \mathrm{~Hz}, 1 \mathrm{H}), 7.29(\mathrm{~m}, 1 \mathrm{H}), 7.13(\mathrm{t}, 7.69 \mathrm{~Hz}, 1 \mathrm{H}), 6.78(\mathrm{~m}, 1 \mathrm{H}), 3.85(\mathrm{bs}$, $2 \mathrm{H}), 3.81(\mathrm{~s}, 3 \mathrm{H}) ;{ }^{13} \mathrm{C}$ NMR $\left(75 \mathrm{MHz}, \mathrm{CDCl}_{3}\right): \delta 167.1,146.5,130.8,129.0,125.2,119.2,115.5$, 51.8. Physical and spectral data were consistent with commercially available material.

Methyl 4-aminobenzoate (14): Subjection of methyl 4-nitrobenzoate $(1 \mathrm{mmol}, 0.181 \mathrm{~g})$ to the general procedure for reducing nitro arenes afforded $0.1511 \mathrm{~g}(100 \%)$ of methyl 4-aminobenzoate (14) as a yellow solid (silica gel FC, hexanes/EtOAc: 80/20 then 50/50); $\mathrm{mp}=108{ }^{\circ} \mathrm{C} .{ }^{1} \mathrm{H}$ NMR $\left(300 \mathrm{MHz}, \mathrm{CDCl}_{3}\right.$ ): $\delta 7.79$ (d, $\left.J=8.24 \mathrm{~Hz}, 2 \mathrm{H}\right), 6.57$ (d, $J=8.79 \mathrm{~Hz}, 2 \mathrm{H}$ ), 4.13 (bs, 2H), 3.79 (s, $3 \mathrm{H}) ;{ }^{13} \mathrm{C}$ NMR $\left(75 \mathrm{MHz}, \mathrm{CDCl}_{3}\right): \delta 167.1,150.9,131.4,119.1,113.5,51.4$. Physical and spectral data were consistent with commercially available material.

2-Aminobenzonitrile (15): Subjection of 2-nitrobenzonitrile (1 $\mathrm{mmol}, 0.148 \mathrm{~g})$ to the general procedure for reducing nitro arenes with stirring for $12 \mathrm{~h}$ afforded $0.1147 \mathrm{~g}(97 \%)$ of 2 aminobenzonitrile (15) as a tan solid (silica gel FC, hexanes/EtOAc: 80/20 then 50/50); mp = 52 ${ }^{\circ} \mathrm{C} .{ }^{1} \mathrm{H}$ NMR $\left(300 \mathrm{MHz}, \mathrm{CDCl}_{3}\right): \delta 7.38-7.22(\mathrm{~m}, 2 \mathrm{H}), 6.69(\mathrm{~m}, 2 \mathrm{H}), 4.42(\mathrm{bs}, 2 \mathrm{H}) ;{ }^{33} \mathrm{C}$ NMR $(75$ $\left.\mathrm{MHz}, \mathrm{CDCl}_{3}\right): \delta 149.6,133.9,132.2,117.7,115.0,95.7$. Physical and spectral data were consistent with commercially available material.

3-Aminobenzonitrile (16): Subjection of 3-nitrobenzonitrile (1 $\mathrm{mmol}, 0.148 \mathrm{~g})$ to the general procedure for reducing nitro arenes with stirring for $12 \mathrm{~h}$ afforded $0.1155 \mathrm{~g}$ (98\%) of 3aminobenzonitrile (16) as a yellow-orange solid (silica gel FC, hexanes/EtOAc: 80/20 then 50/50); $\mathrm{mp}=49-50{ }^{\circ} \mathrm{C} .{ }^{1} \mathrm{H}$ NMR $\left(300 \mathrm{MHz}, \mathrm{CDCl}_{3}\right): \delta 7.16(\mathrm{t}, J=7.96,1 \mathrm{H}), 6.94(\mathrm{~m}, 1 \mathrm{H}), 6.88-6.78(\mathrm{~m}$, $2 \mathrm{H}), 3.85$ (bs, $2 \mathrm{H}) ;{ }^{13} \mathrm{C} \mathrm{NMR}\left(75 \mathrm{MHz}, \mathrm{CDCl}_{3}\right): \delta 146.9,129.8,121.6,119.1,119.1,117.2,112.5$. Physical and spectral data were consistent with commercially available material.

4-Aminobenzonitrile (17): Subjection of 4-nitrobenzonitrile (1 mmol, $0.148 \mathrm{~g})$ to the general procedure for reducing nitro arenes with stirring for $12 \mathrm{~h}$ afforded an inseparable mixture of $0.1043 \mathrm{~g}(78 \%)$ of 4-hydroxyaminobenzonitrile and $0.0094 \mathrm{~g}(8 \%)$ of 4-aminobenzonitrile (17) as a bright yellow solid (silica gel FC, hexanes/EtOAc: 80/20 then 50/50). For 4hydroxyaminobenzonitrile: ${ }^{1} \mathrm{H}$ NMR $\left(300 \mathrm{MHz}, \mathrm{CDCl}_{3}\right): \delta 7.83(\mathrm{~s}, 1 \mathrm{H}), 7.37(\mathrm{~d}, J=8.79 \mathrm{~Hz}, 2 \mathrm{H})$, 7.29 (bs, $1 \mathrm{H}), 6.87$ (d, $J=8.24 \mathrm{~Hz}, 2 \mathrm{H}) ;{ }^{13} \mathrm{C}$ NMR $\left(75 \mathrm{MHz}, \mathrm{CDCl}_{3}\right): \delta 154.5,133.5,132.9,119.7$, $114.1,112.8,102.1$. Physical and spectral data were consistent with those previously reported. ${ }^{10}$

3'-Aminoacetophenone (18): Subjection of 3-nitroacetophenone $(1 \mathrm{mmol}, 0.165 \mathrm{~g})$ to the general procedure for reducing nitro arenes afforded $0.1208 \mathrm{~g}(89 \%)$ of 3 '-aminoacetophenone (18) as a cream yellow solid (silica gel $\mathrm{FC}$, hexanes/EtOAc: $50 / 50) ; \mathrm{mp}=94-95^{\circ} \mathrm{C}$ and $0.0122 \mathrm{~g}$ (7\%) of starting material. For 3'-aminoacetophenone (18): ${ }^{1} \mathrm{H} \mathrm{NMR}\left(300 \mathrm{MHz}, \mathrm{CDCl}_{3}\right): \delta$ 7.29-7.12 (m, 3H), 6.80 (dd, $J=2.19$ and $7.69 \mathrm{~Hz}, 1 \mathrm{H}), 3.86$ (bs, $2 \mathrm{H}), 2.49(\mathrm{~s}, 3 \mathrm{H}) ;{ }^{33} \mathrm{C}$ NMR $(75$

(10) Bordwell, F. G.; Liu, W. J. Am. Chem. Soc. 1996, 118, 8777-8781. 
$\left.\mathrm{MHz}, \mathrm{CDCl}_{3}\right): \delta 198.4,146.7,137.9,129.2,119.5,118.5,113.8,26.5$. Physical and spectral data were consistent with commercially available material.

4'-Aminoacetophenone (19): Subjection of 4-nitroacetophenone $(1 \mathrm{mmol}, 0.165 \mathrm{~g})$ to the general procedure for reducing nitro arenes afforded $0.1291 \mathrm{~g}(95 \%)$ of 4'-aminoacetophenone (19) as a yellow solid; $\mathrm{mp}=102^{\circ} \mathrm{C}$, and $0.0064 \mathrm{~g} \mathrm{(4 \% )}$ of starting material (silica gel FC, hexanes/EtOAc: 80/20 then 50/50). For 4'-aminoacetophenone (19): ${ }^{1} \mathrm{H} \mathrm{NMR}\left(300 \mathrm{MHz}, \mathrm{CDCl}_{3}\right)$ : $\delta 7.84(\mathrm{~d}, J=6.59 \mathrm{~Hz}, 2 \mathrm{H}), 6.69(\mathrm{~d}, J=6.59 \mathrm{~Hz}, 2 \mathrm{H}), 4.47$ (bs, 2H), $2.54(\mathrm{~s}, 3 \mathrm{H}) ;{ }^{13} \mathrm{C}$ NMR $(75$ $\left.\mathrm{MHz}, \mathrm{CDCl}_{3}\right): \delta 196.4,151.0,130.7,127.7,113.6,26.0$. Physical and spectral data were consistent with commercially available material.

4-Methoxyaniline (20): Subjection of 4-nitroanisole $(1 \mathrm{mmol}, 0.153 \mathrm{~g})$ to the general procedure for reducing nitro arenes afforded $0.1209 \mathrm{~g}(98 \%)$ of 4-methoxyaniline (20) as a tan solid (silica gel FC, hexanes/EtOAc: 80/20 then 50/50); $\mathrm{mp}=55-56^{\circ} \mathrm{C}$. ${ }^{1} \mathrm{H} \mathrm{NMR}\left(300 \mathrm{MHz}, \mathrm{CDCl}_{3}\right): \delta 6.73$ (d, $J=8.79 \mathrm{~Hz}, 2 \mathrm{H}), 6.61(\mathrm{~d}, J=8.79 \mathrm{~Hz}, 2 \mathrm{H}), 3.71(\mathrm{~s}, 3 \mathrm{H}), 3.40(\mathrm{bs}, 2 \mathrm{H}) ;{ }^{13} \mathrm{C} \mathrm{NMR}(75 \mathrm{MHz}$, $\left.\mathrm{CDCl}_{3}\right): \delta 152.5,139.8,116.2,114.6,55.6$. Physical and spectral data were consistent with commercially available material.

4-Acetoxyaniline (21): Subjection of 4-acetoxynitrobenzene (1 mmol, $0.181 \mathrm{~g})$ to the general procedure for reducing nitro arenes afforded $0.1427 \mathrm{~g} \mathrm{(94 \% )}$ of 4-acetoxyaniline (21) as a tan-red solid (silica gel FC, hexanes/EtOAc: 80/20, 50/50, then 0/100); $\mathrm{mp}=73{ }^{\circ} \mathrm{C}$. ${ }^{1} \mathrm{H}$ NMR $(300 \mathrm{MHz}$, $\mathrm{CDCl}_{3}$ ): $\delta 6.82(\mathrm{~d}, J=8.79 \mathrm{~Hz}, 2 \mathrm{H}), 6.59(\mathrm{~d}, J=8.79 \mathrm{~Hz}, 2 \mathrm{H}), 3.62(\mathrm{bs}, 2 \mathrm{H}), 2.22(\mathrm{~s}, 3 \mathrm{H}) ;{ }^{13} \mathrm{C}$ NMR $\left(75 \mathrm{MHz}, \mathrm{CDCl}_{3}\right): \delta 170.0,144.2,142.4,121.9,115.3,20.8$. Physical and spectral data were consistent with those previously reported. ${ }^{11}$

4-tert-Butyldimethylsiloxyaniline (22): Subjection of tert-Butyldimethyl-(4-nitro-phenoxy)-silane $(1 \mathrm{mmol}, 0.253 \mathrm{~g})$ to the general procedure for reducing nitro arenes afforded $0.2054 \mathrm{~g}(92 \%)$ of 4-tert-butyldimethylsiloxyaniline (22) as a dark yellow oil and $0.0075 \mathrm{~g}(7 \%)$ of 4-aminophenol (silica gel FC, hexanes/EtOAc: 80/20, 50/50, then 0/100). For 4-tert-butyldimethylsiloxyaniline (22): ${ }^{1} \mathrm{H}$ NMR $\left(300 \mathrm{MHz}, \mathrm{CDCl}_{3}\right): \delta 6.65(\mathrm{~d}, J=8.79 \mathrm{~Hz}, 2 \mathrm{H}), 6.56(\mathrm{~d}, J=8.79 \mathrm{~Hz}, 2 \mathrm{H}), 3.91$ (bs, 2H), $0.94(\mathrm{~s}, 9 \mathrm{H}), 0.13(\mathrm{~s}, 6 \mathrm{H}) ;{ }^{13} \mathrm{C}$ NMR $\left(75 \mathrm{MHz}, \mathrm{CDCl}_{3}\right): \delta 139.7,126.0,120.6,116.5,115.5$, 18.1, -4.5. IR (neat) 3460, 2957, 2930, 2858, 1591, 1510, 1336, 1248, 912, 841, $781 \mathrm{~cm}^{-1}$; LRMS (El, 70 eV) 224 (11), 223 (93), 167 (29), 166 (100), 138 (38), 109 (12), 93 (7), 73 (17), 65 (34).

2-Amino-m-xylene (23): Subjection of 2-nitro- $m$-xylene $(1 \mathrm{mmol}, 0.136 \mathrm{~mL})$ to the general procedure for reducing nitro arenes with 5 equivalents of PMHS $(5 \mathrm{mmol}, 0.3 \mathrm{~mL})$ afforded $0.1211 \mathrm{~g}(100 \%)$ of 2-amino-m-xylene $(\mathbf{2 3})$ as a light yellow oil (silica gel FC, hexanes/EtOAc: 95/5 then 80/20). ${ }^{1} \mathrm{H}$ NMR $\left(300 \mathrm{MHz}, \mathrm{CDCl}_{3}\right): \delta 6.99(\mathrm{~d}, J=7.14 \mathrm{~Hz}, 2 \mathrm{H}), 6.70(\mathrm{t}, J=7.69 \mathrm{~Hz}$, $1 \mathrm{H}), 3.57$ (bs, 2H), 2.23 (s, 6H); ${ }^{13} \mathrm{C}$ NMR $\left(75 \mathrm{MHz}, \mathrm{CDCl}_{3}\right): \delta 142.7,128.1,121.5,117.9,17.5$. Physical and spectral data were consistent with commercially available material.

4-Aminobenzaldehyde (24): Subjection of 4-nitrobenzaldehyde ( $1 \mathrm{mmol}, 0.151 \mathrm{~g})$ to the general procedure for reducing nitro arenes afforded an inseparable mixture of $0.0879 \mathrm{~g}(73 \%)$ of 4 aminobenzaldehyde (24) and $0.0376 \mathrm{~g}(24 \%)$ of (4-nitrophenyl)-methanol as a yellow solid containing residual ethyl acetate (silica gel FC, hexanes/EtOAc: 50/50). Upon complete removal of the ethyl acetate a yellow solid formed, which was insoluble in all solvents tested (presumable from polymerization of the two products). For 4-aminobenzaldehyde (24): ${ }^{1} \mathrm{H} \mathrm{NMR} \mathrm{(300} \mathrm{MHz,}$ $\left.\mathrm{CDCl}_{3}\right): \delta 9.62(\mathrm{~s}, 1 \mathrm{H}), 7.58(\mathrm{~d}, J=6.59 \mathrm{~Hz}, 2 \mathrm{H}), 6.62(\mathrm{~d}, J=6.59 \mathrm{~Hz}, 2 \mathrm{H}), 4.55(\mathrm{bs}, 2 \mathrm{H}) ;{ }^{13} \mathrm{C}$

(11) Bargota, R. S.; Akhtar, M.; Biggadike, K.; Gani, D.; Allemann, R. K. Bioorg. Med. Chem. Lett. 2003, 1623-1626. 
NMR (75 MHz, $\mathrm{CDCl}_{3}$ ): $\delta$ 190.6, 152.8, 132.3, 126.9, 113.8; For (4-nitrophenyl)-methanol: ${ }^{1} \mathrm{H}$ $\operatorname{NMR}\left(300 \mathrm{MHz}, \mathrm{CDCl}_{3}\right): \delta 8.10(\mathrm{~d}, J=6.59 \mathrm{~Hz}, 2 \mathrm{H}), 7.46(\mathrm{~d}, J=7.14 \mathrm{~Hz}, 2 \mathrm{H}), 4.73(\mathrm{~s}, 2 \mathrm{H}), 3.70$ (bs, $1 \mathrm{H}) ;{ }^{13} \mathrm{C}$ NMR $\left(75 \mathrm{MHz}, \mathrm{CDCl}_{3}\right): \delta 148.6,126.8,123.4,63.6$

1-Amino-4-(trifluoromethyl)benzene (25): Subjection of 4-nitrotrifluorotoluene (1 mmol, 0.191 g) to the general procedure for reducing nitro arenes afforded $0.1610 \mathrm{~g}(99 \%, 90 \%$ purity, contaminated with siloxane) of 1-amino-4-(trifluoromethyl)benzene (25) as a yellow oil (silica gel FC, hexanes/EtOAc: $80 / 20$ then 50/50). Pure product could be obtained by following the general procedure for reducing nitro arenes with the following modification. After filtration through the plug of Celite/alumina the filtrate was stirred with $5 \mathrm{~mol} \%$ TBAF for 4 hours. The reaction was concentrated and subjecting to flash chromatography to afford 1-amino-4(trifluoromethyl)benzene that was contaminated with TBA and traces of siloxane (quantitative yield, 95\% purity). Short-path distallation of this crude material afforded 1-amino-4(trifluoromethyl)benzene (25) (91\%); b.p. $75{ }^{\circ} \mathrm{C}$ at $1 \mathrm{~mm} \mathrm{Hg} .{ }^{1} \mathrm{H} \mathrm{NMR}\left(300 \mathrm{MHz}, \mathrm{CDCl}_{3}\right): \delta 7.36$ $(\mathrm{d}, J=8.24 \mathrm{~Hz}, 2 \mathrm{H}), 6.65$ (d, $J=8.24 \mathrm{~Hz}, 2 \mathrm{H}), 3.91$ (bs, $2 \mathrm{H}) ;{ }^{13} \mathrm{C} \mathrm{NMR}\left(75 \mathrm{MHz}, \mathrm{CDCl}_{3}\right): \delta$ 149.4, $126.67,126.62,126.56,126.52,123.0,120.1,119.7,114.1,107.9$. Physical and spectral data were consistent with commercially available material.

4-Nitroaniline (26): Subjection of 1,4-dinitrobenzene $(1 \mathrm{mmol}, 0.168 \mathrm{~g})$ to the general procedure for reducing nitro arenes afforded $0.0994 \mathrm{~g}(72 \%)$ of 4-nitroaniline (26) as an orange solid, $\mathrm{mp}=$ $147-148^{\circ} \mathrm{C}$ and $0.0217 \mathrm{~g}(20 \%)$ of 1,4 -diaminobenzene as a tan solid (silica gel FC, hexanes/EtOAc: 80/20, 50/50, 0/100, then 80/20[EtOAc/MeOH]). For 4-nitroaniline (26): ${ }^{1} \mathrm{H} N M R$ $\left(300 \mathrm{MHz}, \mathrm{CDCl}_{3}+\mathrm{DMSO}-d_{6}\right): \delta 7.68(\mathrm{~d}, J=8.79 \mathrm{~Hz}, 2 \mathrm{H}), 6.32(\mathrm{~d}, J=9.34 \mathrm{~Hz}, 2 \mathrm{H}$ ), 5.27 (bs, $2 \mathrm{H}) ;{ }^{13} \mathrm{C}$ NMR $\left(75 \mathrm{MHz}, \mathrm{CDCl}_{3}+\mathrm{DMSO}_{-} d_{6}\right): \delta 153.7,136.7,125.6,112.2$. Physical and spectral data were consistent with commercially available material.

4-Acetylaminobenzoic acid (27): 4-Nitrobenzoic acid (1 mmol, $0.167 \mathrm{~g})$ was subjected to the general procedure for reducing nitro arenes with the following modification. After complete consumption of the starting material (as judged by TLC) acetic anhydride $(2 \mathrm{mmol}, 0.188 \mathrm{~mL}$ ) was added to the reaction mixture followed by an additional 30 minutes of stirring. Following the general extraction protocol, the organics were filtered though a plug of Celite. Concentration of the crude material and flash chromatography afforded $0.1689 \mathrm{~g}(94 \%)$ of 4-acetylaminobenzoic acid (27) as a white solid (silica gel FC, hexanes/EtOAc: 50/50, 0/100, then 80/20[EtOAc/MeOH]); $\mathrm{mp}=259-262{ }^{\circ} \mathrm{C} .{ }^{1} \mathrm{H}$ NMR $\left(300 \mathrm{MHz}, \mathrm{CDCl}_{3}+\mathrm{DMSO}_{-} \mathrm{d}_{6}\right): \delta 9.98(\mathrm{~s}, 1 \mathrm{H}), 7.90(\mathrm{~d}, J=8.24 \mathrm{~Hz}$, $2 \mathrm{H}), 7.68(\mathrm{~d}, J=8.24 \mathrm{~Hz}, 2 \mathrm{H}), 2.12(\mathrm{~s}, 3 \mathrm{H}) ;{ }^{13} \mathrm{C}$ NMR $\left(75 \mathrm{MHz}, \mathrm{CDCl}_{3}+\mathrm{DMSO}_{-} \mathrm{d}_{6}\right): \delta$ 168.7, 167.2, 142.9, 130.1, 124.9, 118.1, 23.9. Physical and spectral data were consistent with commercially available material.

6-Bromohexyl 4-aminobenzoate (28): Subjection of 6-bromohexyl 4-nitrobenzoate ${ }^{12}(1 \mathrm{mmol}$, $0.333 \mathrm{~g}$ ) to the general procedure for reducing nitro arenes with 3.5 equiv. PMHS (3.5 mmol, 0.21 $\mathrm{mL}$ ) afforded $0.3002 \mathrm{~g}(100 \%)$ of 6-bromohexyl 4-aminobenzoate (28) as a white solid (silica gel FC, hexanes/EtOAc: $95 / 5$ then 50/50); $\mathrm{mp}=77-78{ }^{\circ} \mathrm{C}$. ${ }^{1} \mathrm{H}$ NMR $\left(300 \mathrm{MHz}, \mathrm{CDCl}_{3}\right): \delta 7.79(\mathrm{~d}, \mathrm{~J}=$ $8.79 \mathrm{~Hz}, 2 \mathrm{H}), 6.58(\mathrm{~d}, J=8.79 \mathrm{~Hz}, 2 \mathrm{H}), 4.21$ (t, $J=6.59 \mathrm{~Hz}, 2 \mathrm{H}), 4.07$ (bs, 2H), 3.35 (t, $J=6.59$ $\mathrm{Hz}, 2 \mathrm{H}$ ), 1.82 (quin, $J=6.59 \mathrm{~Hz}, 2 \mathrm{H}$ ), 1.69 (quin, $J=6.59 \mathrm{~Hz}, 2 \mathrm{H}), 1.42(\mathrm{~m}, 4 \mathrm{H}) ;{ }^{13} \mathrm{C}$ NMR $(75$ $\mathrm{MHz}, \mathrm{CDCl}_{3}$ ): $\delta 166.6,150.8,131.3,119.4,113.5,64.1,33.7,32.4,28.4,27.6,25.1$; IR (THF solution) 3445, 3360, 3238, 2937, 1705, 1604, 1518, 1275, 1170, 1113, 844, $773 \mathrm{~cm}^{-1}$; LRMS (El, 70 eV) 300 (12), 299 (11), 138 (12), 137 (100), 80 (7), 79 (100), 92 (40), 65 (15), 64 (14).

(12) See page S10 for preparation. 
4-(Methylthio)aniline (29): Subjection of 4-nitrothioanisole $(1 \mathrm{mmol}, 0.169 \mathrm{~g})$ to the general procedure for reducing nitro arenes afforded $0.150 \mathrm{~g}$ of a brown solid consisting of complex mixture of compounds of which $\sim 10 \%$ was 4 -(methylthio)aniline (29).

3-Aminobenzamide (30): Subjection of 3-nitrobenzamide (1 mmol, $0.166 \mathrm{~g})$ to the general procedure for reducing nitro arenes afforded $0.125 \mathrm{~g}(92 \%)$ of 3-aminobenzamide (30) as a white solid (silica gel FC, EtOAc/MeOH: 100/0 then 80/20); $\mathrm{mp}=117-117.5^{\circ} \mathrm{C}$. ${ }^{1} \mathrm{H} \mathrm{NMR}(300 \mathrm{MHz}$, DMSO- $\left.d_{6}\right): \delta 7.69$ (bs, $\left.1 \mathrm{H}\right), 7.11$ (bs, $\left.1 \mathrm{H}\right), 7.05-6.86(\mathrm{~m}, 3 \mathrm{H}), 6.62(\mathrm{~d}, J=7.69 \mathrm{~Hz}, 1 \mathrm{H}), 5.14$ (bs $2 \mathrm{H}) ;{ }^{13} \mathrm{C}$ NMR $\left(75 \mathrm{MHz}\right.$, DMSO- $\left.d_{6}\right): \delta 168.2,148.6,135.1,128.6,116.5,114.7,113.1$. Physical and spectral data were consistent with commercially available material.

4,4'-Diaminodiphenylmethane (31): Subjection of bis(4-nitrophenyl)methane (1 mmol, $0.258 \mathrm{~g}$ ) to the general procedure for reducing nitro arenes with 8 equiv of PMHS $(8 \mathrm{mmol}, 0.48 \mathrm{~mL})$ and 4 eq. KF (4 mmol, $0.232 \mathrm{~g})$ afforded $0.1849 \mathrm{~g}(93 \%)$ of 4,4'-diaminodiphenylmethane (31) as a yellow-orange solid (silica gel FC, hexanes/EtOAc: 80/20, 50/50, then $0 / 100$ ); $\mathrm{mp}=89^{\circ} \mathrm{C}$. ${ }^{1} \mathrm{H}$ $\operatorname{NMR}\left(300 \mathrm{MHz}, \mathrm{CDCl}_{3}\right): \delta 6.99(\mathrm{~d}, J=8.24 \mathrm{~Hz}, 4 \mathrm{H}), 6.61$ (d, $\left.J=8.24 \mathrm{~Hz}, 4 \mathrm{H}\right), 3.79(\mathrm{~s}, 2 \mathrm{H}), 3.54$ (bs, $4 \mathrm{H}) ;{ }^{13} \mathrm{C}$ NMR $\left(75 \mathrm{MHz}, \mathrm{CDCl}_{3}\right): \delta 144.1,131.7,129.4,115.1,40.0$. Physical and spectral data were consistent with commercially available material.

2-Aminothiophene (32): 2-Nitrothiophene $(1 \mathrm{mmol}, 0.129 \mathrm{~g})$ was subjected to the general procedure for reducing nitro arenes with the following modification. After complete consumption of the starting material (as judged by TLC) di(tert-butyl)dicarbonate $(2 \mathrm{mmol}, 0.436 \mathrm{~g})$ dissolved in $0.5 \mathrm{~mL}$ THF was injected into the reaction followed by an additional 4 hours of stirring. Following the general extraction protocol, the organics were filtered though a plug of Celite. Concentration of the crude material and flash chromatography afforded $0.1614 \mathrm{~g}(81 \%)$ of tert-butyl thiophen-2ylcarbamate as a white solid (silica gel FC, hexanes/EtOAc: $95 / 5$ then $80 / 20$ ); $\mathrm{mp}=150{ }^{\circ} \mathrm{C} .{ }^{1} \mathrm{H}$ $\operatorname{NMR}\left(300 \mathrm{MHz}, \mathrm{CDCl}_{3}\right): \delta 6.91$ (bs, $\left.1 \mathrm{H}\right), 6.78(\mathrm{~m}, 2 \mathrm{H}), 6.49(\mathrm{dd}, J=1.64,3.29 \mathrm{~Hz}, 1 \mathrm{H}), 1.49(\mathrm{~s}$, $9 \mathrm{H}) ;{ }^{13} \mathrm{C}$ NMR $\left(75 \mathrm{MHz}, \mathrm{CDCl}_{3}\right): \delta 152.4,140.2,124.3,116.9,111.1,81.3,28.2$. Physical and spectral data were consistent with those previously reported. ${ }^{13}$ (2-aminothiophene decomposes immediately when exposed to air, so the reaction system should not be opened to the air until the amine is protected.)

6-Amino-1H-benzimidazole (33): A dry $25 \mathrm{~mL}$ round bottom was charged with $\mathrm{Pd}(\mathrm{OAc})_{2}(0.05$ $\mathrm{mmol}, 0.011 \mathrm{~g})$, sealed, and placed under a positive pressure of nitrogen. Dry THF $(5 \mathrm{~mL})$ and aqueous $\mathrm{KF}$ ( $2 \mathrm{mmol}, 0.116 \mathrm{~g}$, in $2 \mathrm{~mL}$ of degassed water) were added sequentially. PMHS $(0.67$ $\mathrm{mmol}, 0.04 \mathrm{~mL}$ ) was added dropwise via syringe and the reaction was stirred $\sim 15-45$ seconds to preform the PMHS-Pd(OAc) $)_{2}$ nanoparticles. The reaction was then opened (to the air) and 6nitro-1H-benzimidazole $(1 \mathrm{mmol}, 0.163 \mathrm{~g})$ was added quickly in a single portion. The reaction flask was resealed and purged with nitrogen and the remaining PMHS $(3.3 \mathrm{mmol}, 0.20 \mathrm{~mL})$ was added dropwise. The nitrogen inlet was replaced by a balloon of nitrogen. The reaction was stirred for $30 \mathrm{~min}$. Following the general extraction protocol, the organics were filtered though a plug of Celite. Concentration of the crude material and flash chromatography afforded $0.1331 \mathrm{~g}$ (89\%, containing residual $\left.(\sim 10 \%) \mathrm{H}_{2} \mathrm{O}\right)$ of 6 -amino- $1 \mathrm{H}$-benzimidazole (33) as a cream colored solid (silica gel FC, EtOAc/MeOH: $100 / 0,80 / 20$, then 50/50); $\mathrm{mp}=160-165^{\circ} \mathrm{C} .{ }^{1} \mathrm{H}$ NMR $(300$ $\left.\mathrm{MHz}, \mathrm{DMSO}-d_{6}\right): \delta 11.85(\mathrm{bs}, 1 \mathrm{H}), 7.92(\mathrm{~s}, 1 \mathrm{H}), 7.29(\mathrm{~d}, J=8.24 \mathrm{~Hz}, 1 \mathrm{H}), 6.71(\mathrm{~s}, 1 \mathrm{H}), 6.54(\mathrm{~d}, J$ $=8.24 \mathrm{~Hz}, 1 \mathrm{H}), 4.91$ (bs, 2H); ${ }^{13} \mathrm{C}$ NMR $\left(75 \mathrm{MHz}\right.$, DMSO- $\left.d_{6}\right): \delta 144.5,139.6,136.9,132.9,117.1$, 111.6, 96.8. IR (MeOH solution) 3175, 2820, 1635, 1522, 1491, 1361, 1265, 1209, 1028, 949, $812,619 \mathrm{~cm}^{-1}$; LRMS (EI, $\left.70 \mathrm{eV}\right)$ 132, 116, 106, 105, 78, 66, 52. Physical and spectral data were

(13) Greck, C.; Bischoff, L.; Girard, A.; Hajicek, J.; Genêt, J. P. Bull. Soc. Chim. Fr. 1994, 131, 429-433. 
consistent with those previously reported. ${ }^{14}$ (Note: In lieu of opening the reaction system to the air 6-nitro-1H-benzimidazole can be dissolved in a THF $(2 \mathrm{~mL})$ water $(1 \mathrm{~mL})$ mixture and added to the reaction via syringe.)

Methyl 5-amino-2-furoate (34): Subjection of methyl 5-nitro-2-furoate $(1 \mathrm{mmol}, 0.171 \mathrm{~g})$ to the general procedure for reducing nitro arenes afforded $0.124 \mathrm{~g}(89 \%)$ of methyl 5-amino-2-furoate (34) as a yellow solid (silica gel FC, hexanes/EtOAc: $80 / 20$ then $50 / 50$ ); $\mathrm{mp}=134-135^{\circ} \mathrm{C} .{ }^{1} \mathrm{H}$ $\operatorname{NMR}\left(300 \mathrm{MHz}, \mathrm{CDCl}_{3}\right): \delta 7.02(\mathrm{~d}, J=3.29 \mathrm{~Hz}, 1 \mathrm{H}), 6.41(\mathrm{bs}, 2 \mathrm{H}), 5.01(\mathrm{~d}, J=3.29 \mathrm{~Hz}, 1 \mathrm{H}), 3.61$ (s, 3H); ${ }^{13} \mathrm{C}$ NMR $\left(75 \mathrm{MHz}, \mathrm{CDCl}_{3}\right): \delta 161.7,158.0,132.2,123.6,84.2,50.4$. Physical and spectral data were consistent with those previously reported. ${ }^{15}$

$N$-Decylhydroxylamine (35): Subjection of 1-nitrodecane (1 mmol, $0.187 \mathrm{~g})$ to the general procedure for reducing aliphatic nitro compounds afforded $0.1447 \mathrm{~g} \mathrm{(83 \% )}$ of $\mathrm{N}$ decylhydroxylamine (35) as a white solid (silica gel FC, hexanes/EtOAc: $90 / 10$ then 0/100); m.p. $=79{ }^{\circ} \mathrm{C} .{ }^{1} \mathrm{H}$ NMR $\left(300 \mathrm{MHz}, \mathrm{CDCl}_{3}\right): \delta 5.24(\mathrm{bs}, 2 \mathrm{H}), 2.90(\mathrm{t}, J=7.14 \mathrm{~Hz}, 2 \mathrm{H}), 1.49(\mathrm{~m}, 2 \mathrm{H}), 1.22$ $(\mathrm{m}, 14 \mathrm{H}), 0.84(\mathrm{t}, J=7.14 \mathrm{~Hz}, 3 \mathrm{H}) ;{ }^{13} \mathrm{C} \mathrm{NMR}\left(75 \mathrm{MHz}, \mathrm{CDCl}_{3}\right): \delta 53.9,31.9,29.5,29.3,27.1$, 26.9, 22.6, 14.1; IR (KBr) 3270, 3159, 2931, 2865, 1473, 1153, 1061, 891, $721 \mathrm{~cm}^{-1}$; LRMS (El, $70 \mathrm{eV}) 173$ (3), 157 (3), 142 (1), 70 (19), 46 (100).

$\mathrm{N}$-Phenethylhydroxylamine (36): Subjection of (2-nitroethyl)benzene $(1 \mathrm{mmol}, 0.151 \mathrm{~g})$ to the general procedure for reducing aliphatic nitro compounds afforded $0.0803 \mathrm{~g} \mathrm{(58 \% )}$ of $\mathrm{N}$ phenethylhydroxylamine (36) as a white solid (silica gel FC, EtOAc/MeOH: 100/0 then 80/20); m.p. $=85^{\circ} \mathrm{C} .{ }^{1} \mathrm{H}$ NMR $\left(300 \mathrm{MHz}, \mathrm{CDCl}_{3}\right): \delta 7.29(\mathrm{~m}, 2 \mathrm{H}), 7.22(\mathrm{~m}, 3 \mathrm{H}), 6.33(\mathrm{bs}, 2 \mathrm{H}), 3.17(\mathrm{t}, \mathrm{J}=$ $7.14 \mathrm{~Hz}, 2 \mathrm{H}), 2.87$ (t, $J=7.14 \mathrm{~Hz}, 2 \mathrm{H}) ;{ }^{13} \mathrm{C}$ NMR $\left(75 \mathrm{MHz}, \mathrm{CDCl}_{3}\right): \delta 139.0,128.7,128.5,126.2$, 54.7, 33.1; IR (neat) 3246, 3152, 2862, 1496, 1454, 1059, 740, $696 \mathrm{~cm}^{-1}$; LRMS (EI, $\left.70 \mathrm{eV}\right) 137$ (1), 136 (1), 121 (8), 105 (13), 104 (24), 92 (83), 91 (100), 77 (13), 76 (17), 46 (32), 45 (34).

$\mathbf{N}$-hydroxylaminocyclohexane (37): Nitrocyclohexane $(1 \mathrm{mmol}, 0.122 \mathrm{~mL})$ was subjected to the general procedure for reducing aliphatic nitro compounds with the following modification. After complete consumption of the starting material (as judged by TLC) 2 equiv $p$-toluenesulfonic anhydride $(2 \mathrm{mmol}, 0.652 \mathrm{~g})$ was added to the reaction followed by an additional 2 hours of stirring to afford after the general workup $0.2388 \mathrm{~g}(89 \%)$ of $\mathrm{N}$-cyclohexyl- $\mathrm{N}$-hydroxy-4-methylbenzenesulfonamide as a white solid; $\mathrm{mp}=127-129^{\circ} \mathrm{C}$. ${ }^{1} \mathrm{H} \mathrm{NMR}\left(300 \mathrm{MHz}, \mathrm{CDCl}_{3}\right): \delta 7.81(\mathrm{~d}, J$ $=8.24 \mathrm{~Hz}, 2 \mathrm{H}), 7.29(\mathrm{~d}, J=8.24 \mathrm{~Hz}, 2 \mathrm{H}), 6.64(\mathrm{~s}, 1 \mathrm{H}), 3.62(\mathrm{tt}, J=3.84,10.98 \mathrm{~Hz}, 1 \mathrm{H}), 2.41(\mathrm{~s}$, $3 \mathrm{H}), 1.72-0.85(\mathrm{~m}, 10 \mathrm{H}) ;{ }^{13} \mathrm{C} \mathrm{NMR}\left(75 \mathrm{MHz}, \mathrm{CDCl}_{3}\right): \delta 144.3,133.8,129.5,128.8,60.3,28.9$, 25.3, 25.2, 21.6; IR (PTFE card) 3366, 2930, 2855, 1334, 1185, 1082, 665, $584 \mathrm{~cm}^{-1}$; LRMS (El, 70 eV) 269 (1), 253 (3), 157 (78), 139 (46), 91 (100), 83 (44), 77 (7), 76 (9), 55 (46), 54 (44), 41 (53).

1,3-Diacetoxy-2-acetoxymethyl-2-(N-hydroxy)aminopropane (38): Subjection of 1,3diacetoxy-2-acetoxymethyl-2-nitropropane $(1 \mathrm{mmol}, 0.277 \mathrm{~g})$ to the general procedure for reducing aliphatic nitro compounds afforded $0.0819 \mathrm{~g} \mathrm{(31 \% )} \mathrm{of} \mathrm{1,3-Diacetoxy-2-acetoxymethyl-2-}$ ( $N$-hydroxy)aminopropane (38) as a clear oil. ${ }^{1} \mathrm{H}$ NMR (300 MHz, $\mathrm{CDCl}_{3}$ ): $\delta 5.21$ (bs, 2H), 4.14 (s, $6 \mathrm{H}), 2.05$ (s, 9H); ${ }^{13} \mathrm{C}$ NMR $\left(75 \mathrm{MHz}, \mathrm{CDCl}_{3}\right.$ ): $\delta$ 170.8, 62.0, 61.0, 20.7; IR (neat) 3372, 2963, 1734, 1653, 1437, 1379, 1226, 1047, 952, $908 \mathrm{~cm}^{-1}$; LRMS (El, $\left.70 \mathrm{eV}\right) 263$ (0.5), 232 (40), 190 (30), 172 (9), 113 (74), 102 (13), 54 (7), 43 (100).

Methyl 4-( $N$-hydroxy)amino-4-methylpentanoate (40): Subjection of methyl 4-methyl-4nitropentanoate (39) $(1 \mathrm{mmol}, 0.157 \mathrm{~mL})$ to the general procedure for reducing aliphatic nitro

(14) Kihel, A. E.; Benchidmi, M.; Essassi, E. M.; Bauchat, P.; Danion-Bougot, R. Synth. Commun. 1999, $29,2435-2445$.

(15) Padwa, A.; Dimitroff, M.; Waterson, A. G.; Wu T. J. Org. Chem. 1997, 62, 4088-4096. 
compounds afforded trace amounts $(\sim 1 \mathrm{mg})$ of methyl 4-( $N$-hydroxy)amino-4-methylpentanoate along with $0.1119 \mathrm{~g}(64 \%)$ of starting material recovered.

3-Hydroxy-4-methyl-5-phenethyloxazolidin-2-one (42): 4-Nitro-1-phenyl-3-pentanol (41) (1 $\mathrm{mmol}, 0.209 \mathrm{~g}$, syn/anti mixture $=1.77 / 1$ ) was subjected to the general procedure for reducing aliphatic nitro compounds with the following modification. After stirring for 4 hours the starting material was completely consumed (as judged by TLC). At that time 2 equiv of $N, N^{\prime}$ carbonyldiimidazole $(2 \mathrm{mmol}, 0.324 \mathrm{~g}$ ) was added and the reaction was stirred an additional 6 hours to afford after the general workup $0.1822 \mathrm{~g}(89 \%)$ of 3-hydroxy-4-methyl-5phenethyloxazolidin-2-one (42) (anti/syn $=1.8 / 1)$ as clear solid. ${ }^{1} \mathrm{H} \mathrm{NMR}\left(300 \mathrm{MHz}, \mathrm{CDCl}_{3}\right)$ : $\delta$ 8.76 (bs, $1 \mathrm{H}), 7.32-7.12(\mathrm{~m}, 5 \mathrm{H}), 4.43$ (ddd, $J=3.84,7.14,10.43 \mathrm{~Hz}, 0.35 \mathrm{H}), 3.96(\mathrm{~m}, 1 \mathrm{H}), 3.55$ (dq, $J=2.19,6.04 \mathrm{~Hz}, 0.63 \mathrm{H}), 2.85(\mathrm{~m}, 1 \mathrm{H}), 2.66(\mathrm{~m}, 1 \mathrm{H}), 2.10-1.73(\mathrm{~m}, 2 \mathrm{H}), 1.29(\mathrm{~d}, J=6.04$ $\mathrm{Hz}, 2.04 \mathrm{H}), 1.20(\mathrm{~d}, J=6.59 \mathrm{~Hz}, 1.14 \mathrm{H}) ;{ }^{13} \mathrm{C} \mathrm{NMR}\left(75 \mathrm{MHz}, \mathrm{CDCl}_{3}\right): \delta 160.5,160.4,140.2,140.1$, 128.5, 128.4, 128.3, 126.2, 79.8, 76.0, 60.5, 57.8, 34.5, 31.3, 30.9, 16.0; IR (neat) 3267, 2936, 1757, 1496, 1456, 1387, 1228, 1111, 1035, 752, $700 \mathrm{~cm}^{-1}$; LRMS (El, $\left.70 \mathrm{eV}\right) 221$ (20), 220 (13), 204 (12), 146 (9), 116 (39), 105 (44), 104 (67), 99 (15), 91 (100), 77 (50), 41 (100). Spectral data were similar to those previously reported for the oxazolidinone. ${ }^{16}$

3-Phenyl-3,3a,4,5,6,7-hexahydro-2H-indole-1-oxide (4 4 ): Subjection of 2-(2-nitro-1-phenylethyl)-cyclohexanone $(43)(1 \mathrm{mmol}, 0.247 \mathrm{~g})$ to the general procedure for reducing aliphatic nitro compounds with 10 equiv triethylsilane $(10 \mathrm{mmol}, 1.6 \mathrm{~mL})$ afforded $0.165 \mathrm{~g}(77 \%)$ of the nitrone 3-phenyl-3,3a, 4,5,6,7-hexahydro-2 $\mathrm{H}$-indole-1-oxide (44) as an amber oil (silica gel FC, EtOAc/MeOH: 100/0, 90/10, the 80/20). $\left.{ }^{1} \mathrm{H} \mathrm{NMR} \mathrm{(300} \mathrm{MHz,} \mathrm{CDCl}_{3}\right): \delta 7.36-7.11(\mathrm{~m}, 5 \mathrm{H})$, 4.30-4.03 (m, 2H), $3.18(\mathrm{~m}, 2 \mathrm{H}), 2.76(\mathrm{~m}, 1 \mathrm{H}), 2.12-1.85(\mathrm{~m}, 3 \mathrm{H}), 1.79(\mathrm{~m}, 1 \mathrm{H}), 1.45-1.09(\mathrm{~m}$, $3 \mathrm{H}) ;{ }^{13} \mathrm{C}$ NMR $\left(75 \mathrm{MHz}, \mathrm{CDCl}_{3}\right): \delta 148.9,139.7,128.9,127.3,127.1,68.1,50.5,45.7,32.2,24.1$, 23.7, 23.4; IR (neat) 3391, 2937, 2860, 2206, 1624, 1498, 1448, 1379, 1251, 1230, 1180, 925, $731 \mathrm{~cm}^{-1}$; LRMS (EI, $\left.70 \mathrm{eV}\right) 215$ (64), 198 (18), 111 (73), 104 (18), 91 (38), 84 (23), 77 (23). Spectral data were similar to those previously reported for a related nitrone. ${ }^{17}$

Preparation of 6-bromohexyl 4-nitrobenzoate: A flame dried $250 \mathrm{~mL}$ round bottom was charged with 4-nitrobenzoic acid $(60 \mathrm{mmol}, 10 \mathrm{~g})$, 1,6-hexanediol $(180 \mathrm{mmol}, 21.3 \mathrm{~g})$, DMAP (12 mmol, $1.47 \mathrm{~g}$ ), and $60 \mathrm{~mL}$ of dry $\mathrm{CH}_{2} \mathrm{Cl}_{2}$. The round bottom was sealed and purged with nitrogen. The flask was placed in an ice-bath and DCC $\left(1 \mathrm{M}\right.$ in $\left.\mathrm{CH}_{2} \mathrm{Cl}_{2}, 99 \mathrm{mmol}, 99 \mathrm{~mL}\right)$ was added dropwise via a cannula. After complete addition of the DCC the reaction was stirred for an additional 20 minutes at the ice-bath temperature. The reaction was then allowed to warm to room temperature and stir over night $(12 \mathrm{~h})$. The reaction mixture was filtered and the solid material rinsed with $\mathrm{CH}_{2} \mathrm{Cl}_{2}$. The filtrate was washed with $0.5 \mathrm{~N} \mathrm{HCl}$ and then saturated sodium bicarbonate solution. The organics were dried $\left(\mathrm{MgSO}_{4}\right)$, concentrated, and subjected to flash chromatography (gradient hexanes/EtOAc: 90/10, 80/20, 50/50) affording $13.63 \mathrm{~g}(85 \%)$ of 6hydroxyhexyl 4-nitrobenzoate as a pale yellow oil. ${ }^{1} \mathrm{H} \mathrm{NMR}\left(300 \mathrm{MHz}, \mathrm{CDCl}_{3}\right): \delta 8.24(\mathrm{~d}, J=8.79$ $\mathrm{Hz}, 2 \mathrm{H}$ ), 8.15 (d, $J=8.79 \mathrm{~Hz}, 2 \mathrm{H}$ ), 4.30 (t, $J=6.59 \mathrm{~Hz}, 2 \mathrm{H}$ ), 3.61 (t, $J=6.59 \mathrm{~Hz}, 2 \mathrm{H}$ ), 1.70 (quin, $J=6.59 \mathrm{~Hz}, 2 \mathrm{H}$ ), 1.56 (quin, $J=6.59 \mathrm{~Hz}, 2 \mathrm{H}), 1.43(\mathrm{~m}, 4 \mathrm{H}) ;{ }^{13} \mathrm{C} \mathrm{NMR}\left(75 \mathrm{MHz}, \mathrm{CDCl}_{3}\right): \delta 164.7$, $150.4,135.7,130.5,123.4,65.8,62.6,32.4,28.4,25.7,25.3$. This material $(10 \mathrm{mmol}, 2.67 \mathrm{~g})$ was added to a dry $25 \mathrm{~mL}$ round bottom that had been charged with carbon tetrabromide (12.5 mmol, $4.14 \mathrm{~g}$ ) and $15 \mathrm{~mL}$ of dry $\mathrm{CH}_{2} \mathrm{Cl}_{2}$. The reaction was placed in an ice-bath and a nitrogen line was placed in the neck of the round bottom and triphenylphosphine $(15 \mathrm{mmol}, 3.93 \mathrm{~g})$ was added in four portions over a period of 5 minutes. The reaction turned from a light yellow to a

(16) Sasai, H.; Tokunaga, T.; Watanabe, S.; Suzuki, T.; Itoh, N.; Shibasaki, M. J. Org. Chem. 1995, 60, 7388-7389.

(17) (a) Degnan, A. P.; Meyers, A. I. J. Org. Chem. 2000, 65, 3503-3512. (b) For an uncharacterized report of 43 see Klutchko, S.; Sonntag, A. C.; Shavel, J., Jr. 3-Phenylhydroindoles U.S. Patent 3,989,714, November 2, 1976. 
brown orange color after complete addition of the $\mathrm{PPh}_{3}$. The reaction was stirred for 15 minutes, followed by evaporation to half volume. The mixture was then diluted with ether. The solid material was filtered off and rinsed with ether. The filtrate was concentrated and subjected to flash chromatography (hexanes/EtOAc: 90/10) affording $3.12 \mathrm{~g}(94 \%)$ of 6-bromohexyl 4nitrobenzoate as light yellow solid; $\mathrm{mp}=45-47^{\circ} \mathrm{C},{ }^{1} \mathrm{H} \mathrm{NMR}\left(300 \mathrm{MHz}, \mathrm{CDCl}_{3}\right): \delta 8.25(\mathrm{~d}, J=8.79$ $\mathrm{Hz}, 2 \mathrm{H}$ ), $8.17(\mathrm{~d}, J=8.79 \mathrm{~Hz}, 2 \mathrm{H}$ ), $4.34(\mathrm{t}, J=6.59 \mathrm{~Hz}, 2 \mathrm{H}$ ), $3.39(\mathrm{t}, J=6.59 \mathrm{~Hz}, 2 \mathrm{H}$ ), 1.86 (quin, $J=7.14 \mathrm{~Hz}, 2 \mathrm{H}$ ), 1.78 (quin, $J=7.14 \mathrm{~Hz}, 2 \mathrm{H}$ ), $1.56-1.38(\mathrm{~m}, 4 \mathrm{H}) ;{ }^{13} \mathrm{C} \mathrm{NMR}\left(75 \mathrm{MHz}, \mathrm{CDCl}_{3}\right): \delta$ 164.6, 150.4, 135.6, 130.6, 123.5, 65.7, 33.6, 32.4, 28.4, 27.7, 25.1; IR (THF solution) 3111, $3080,3055,2939,1724,1608,1529,1464,1350,1275,1103,1014,873,721 \mathrm{~cm}^{-1}$; LRMS (El, $70 \mathrm{eV}) 250$ (0.13), 167 (12), 164 (33), 162 (13), 161 (19), 150 (35), 149 (46), 133 (11), 135 (8), 137 (9), 120 (20), 104 (16), 103 (21), 92 (14), 82 (100), 75 (18), 76 (18), 67 (14), 55 (30), 54 (26), $41(24)$. 


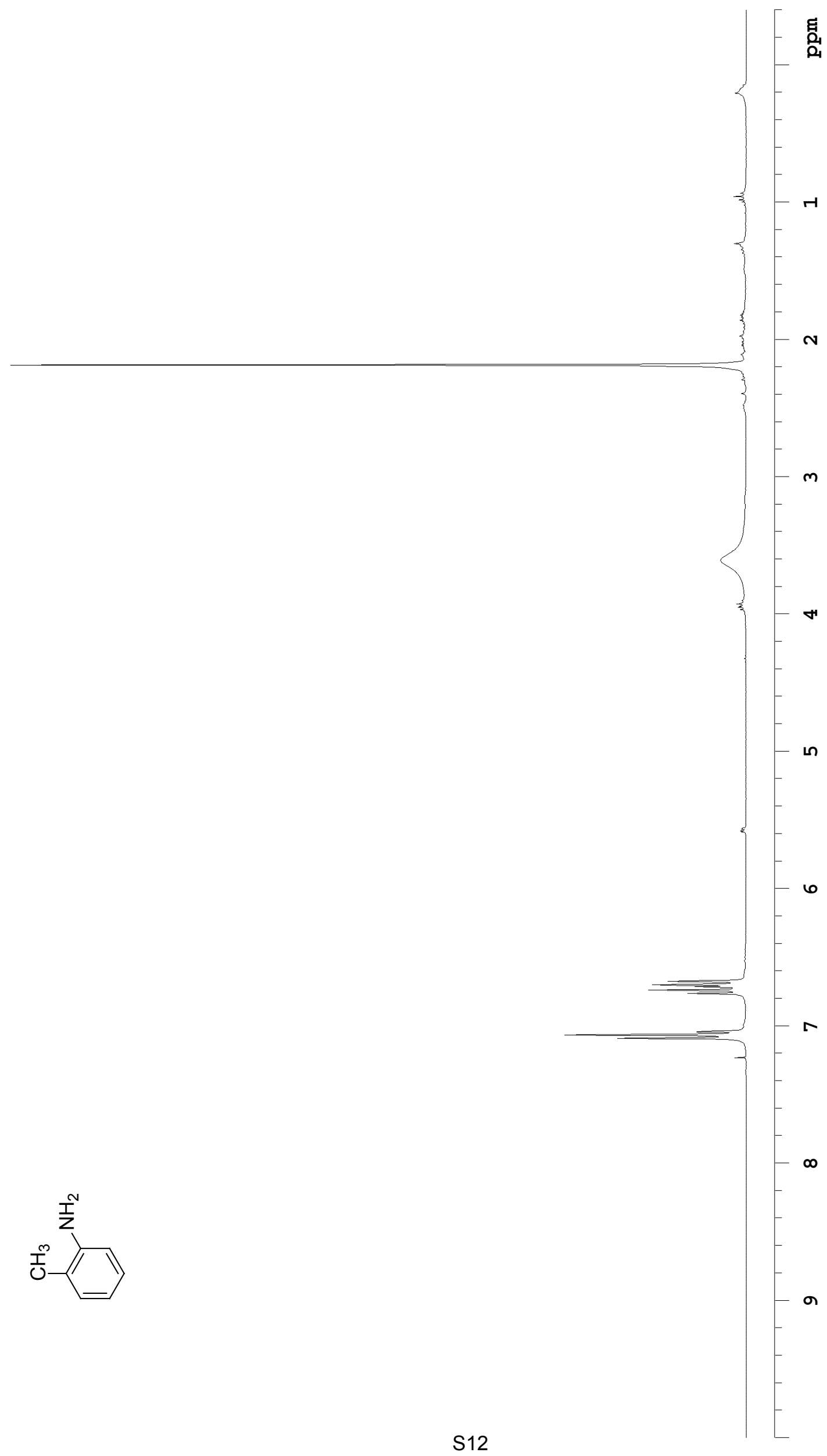




$$
1
$$




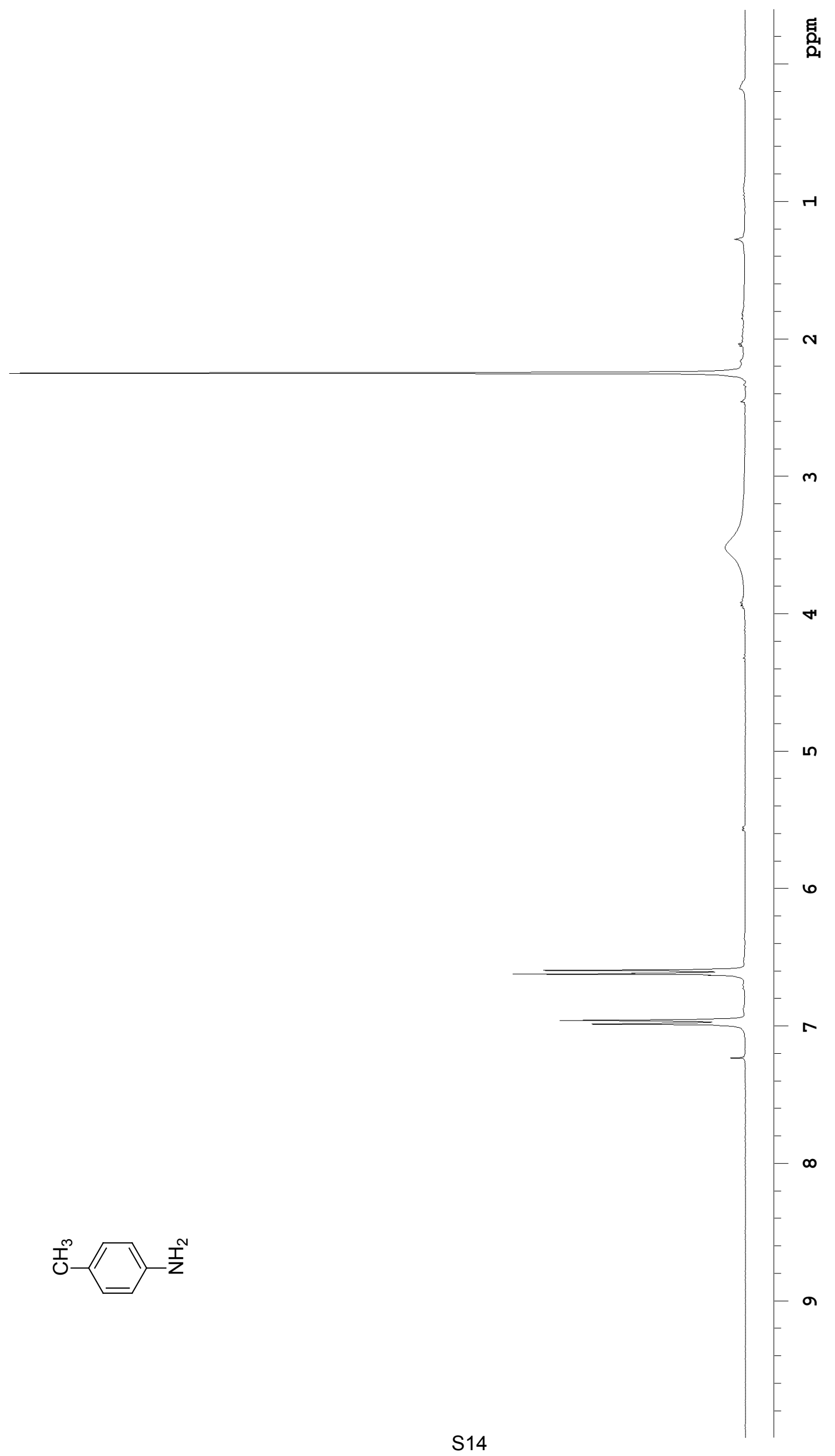




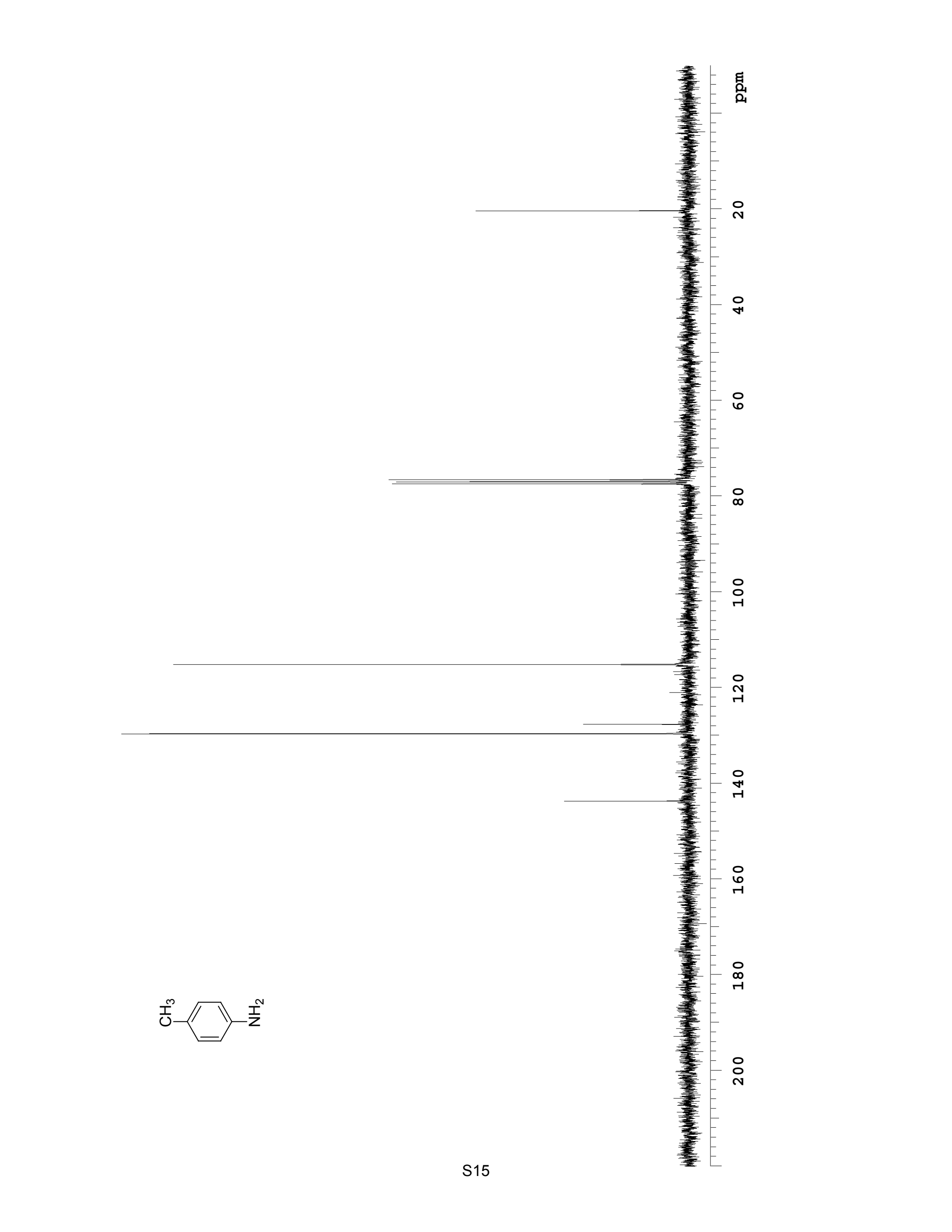




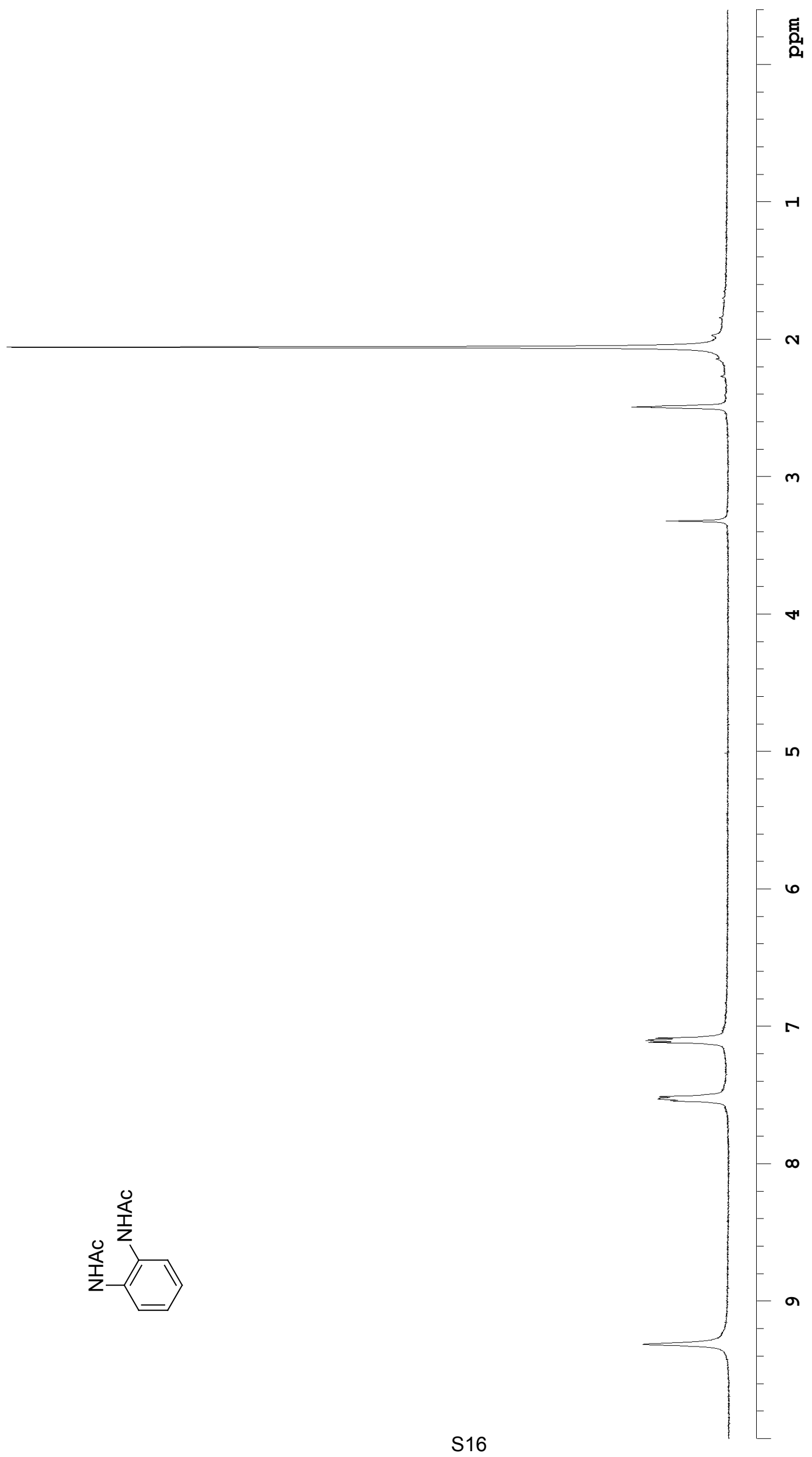




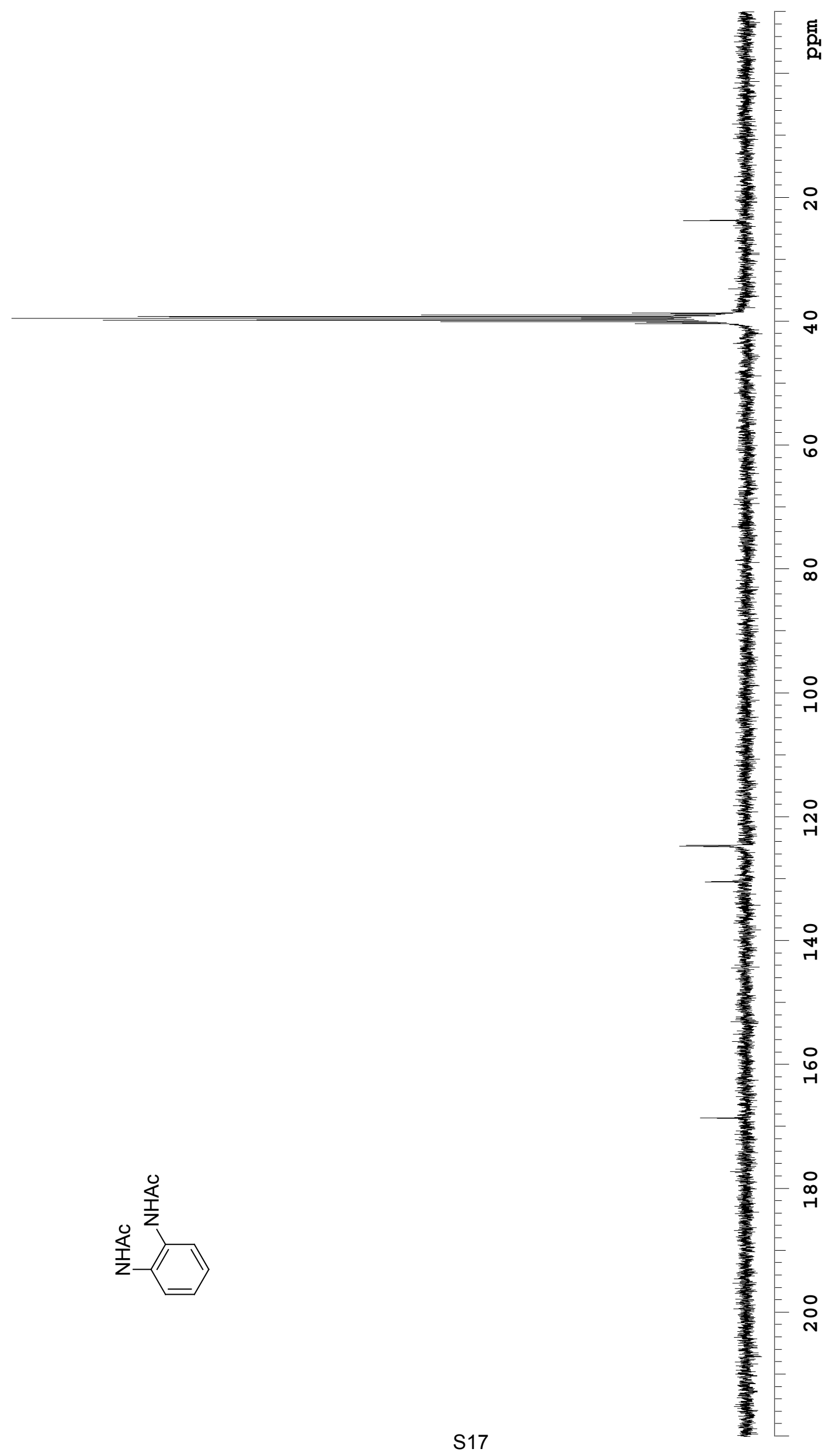




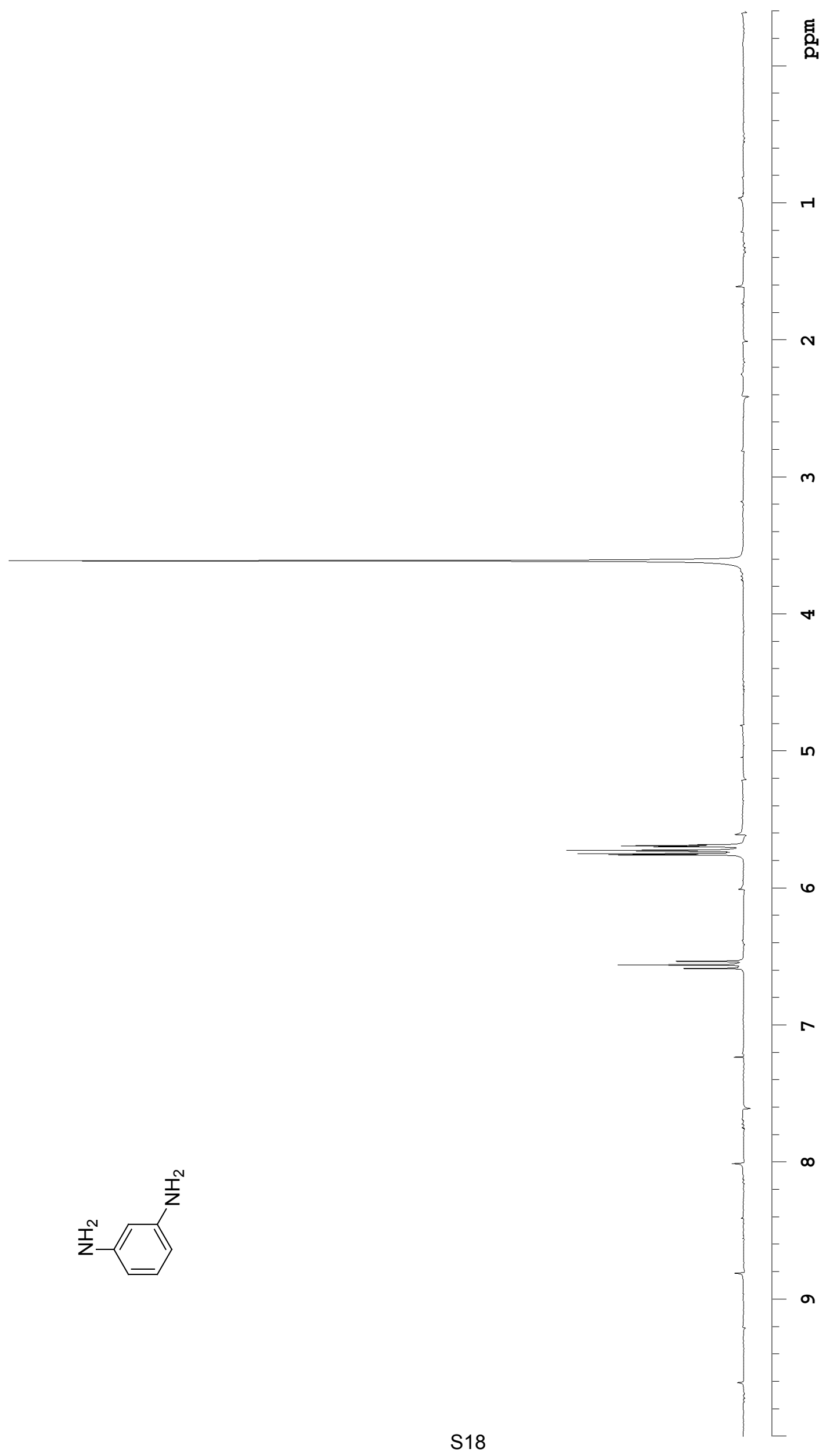




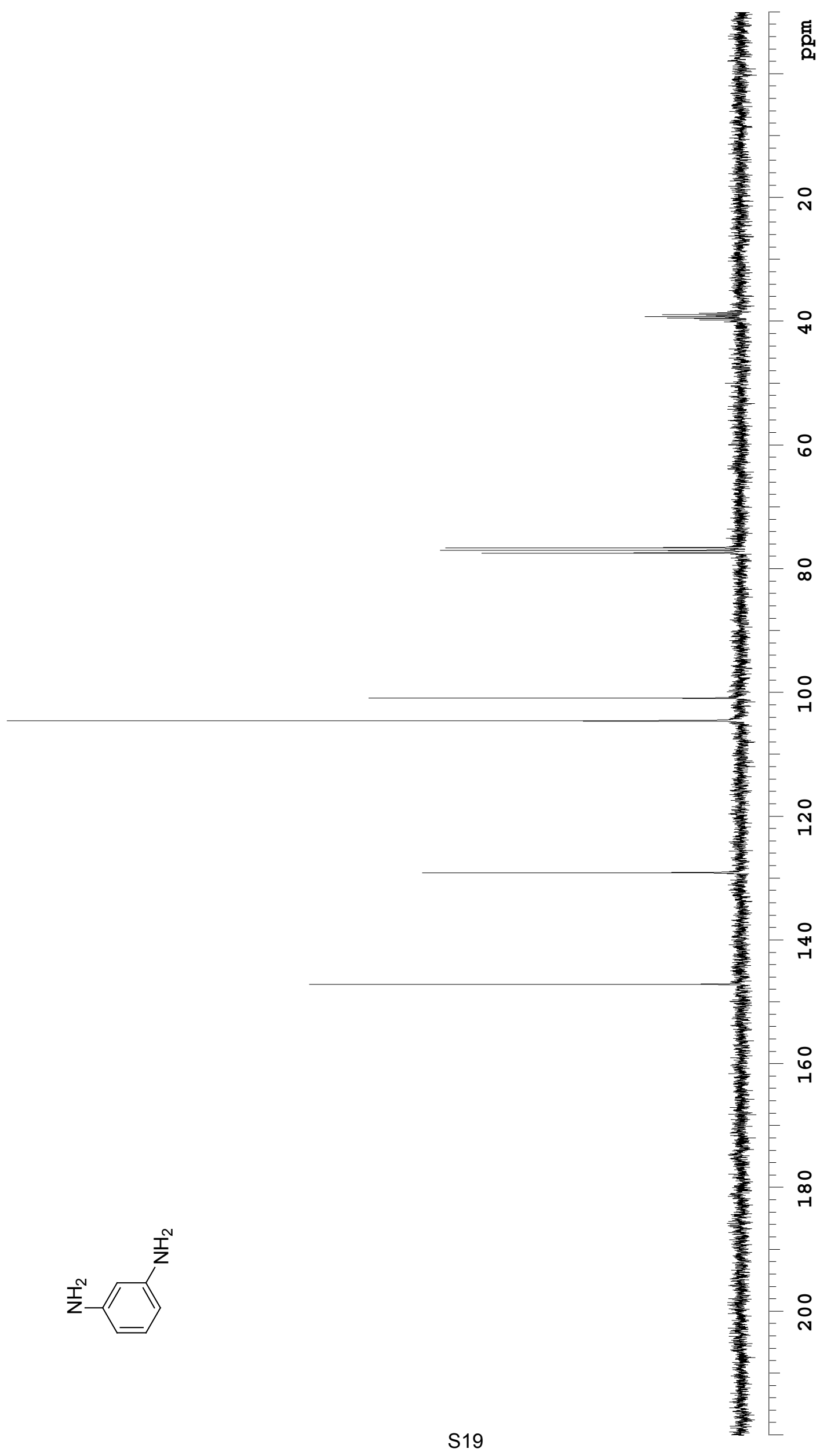




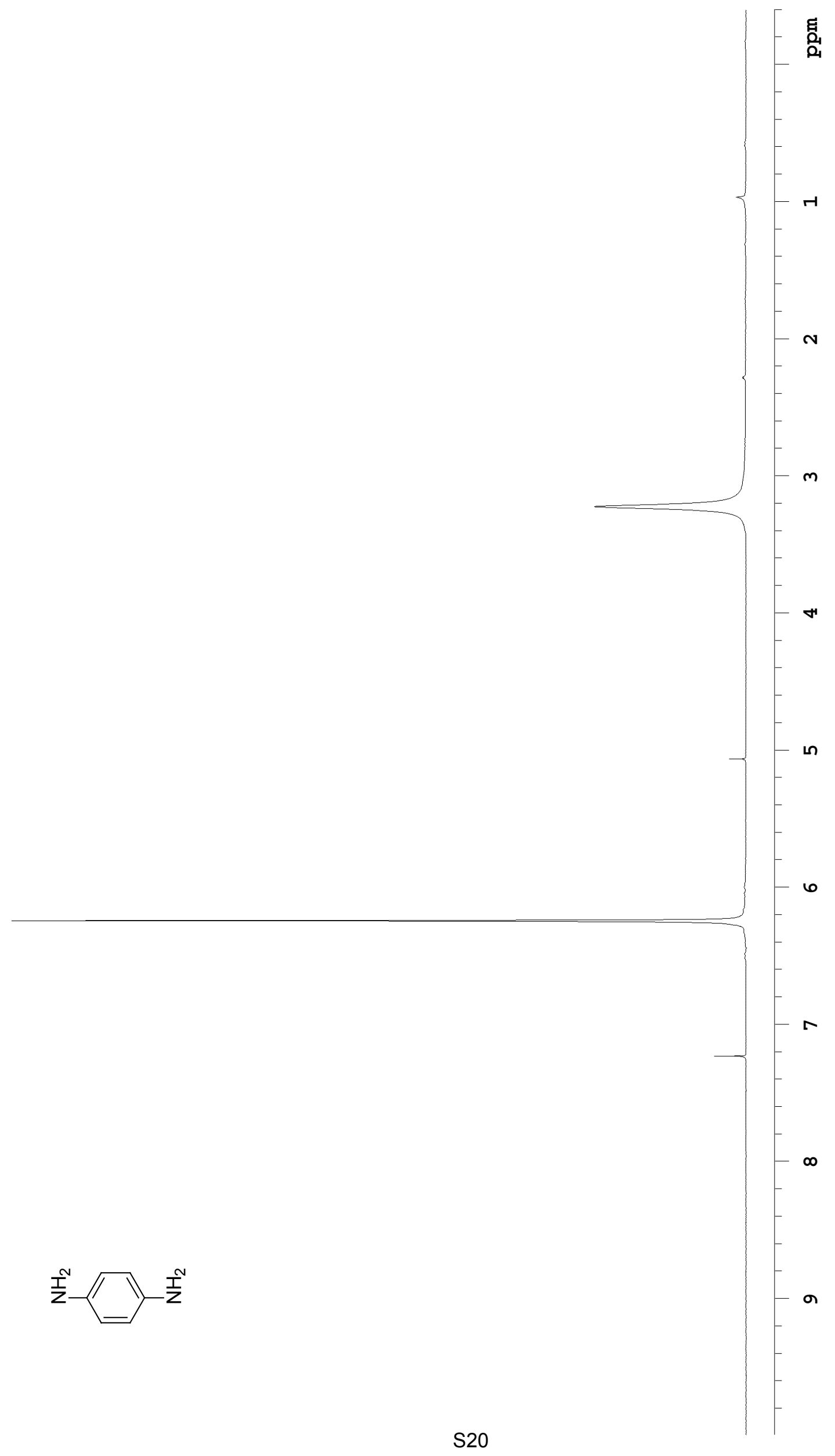




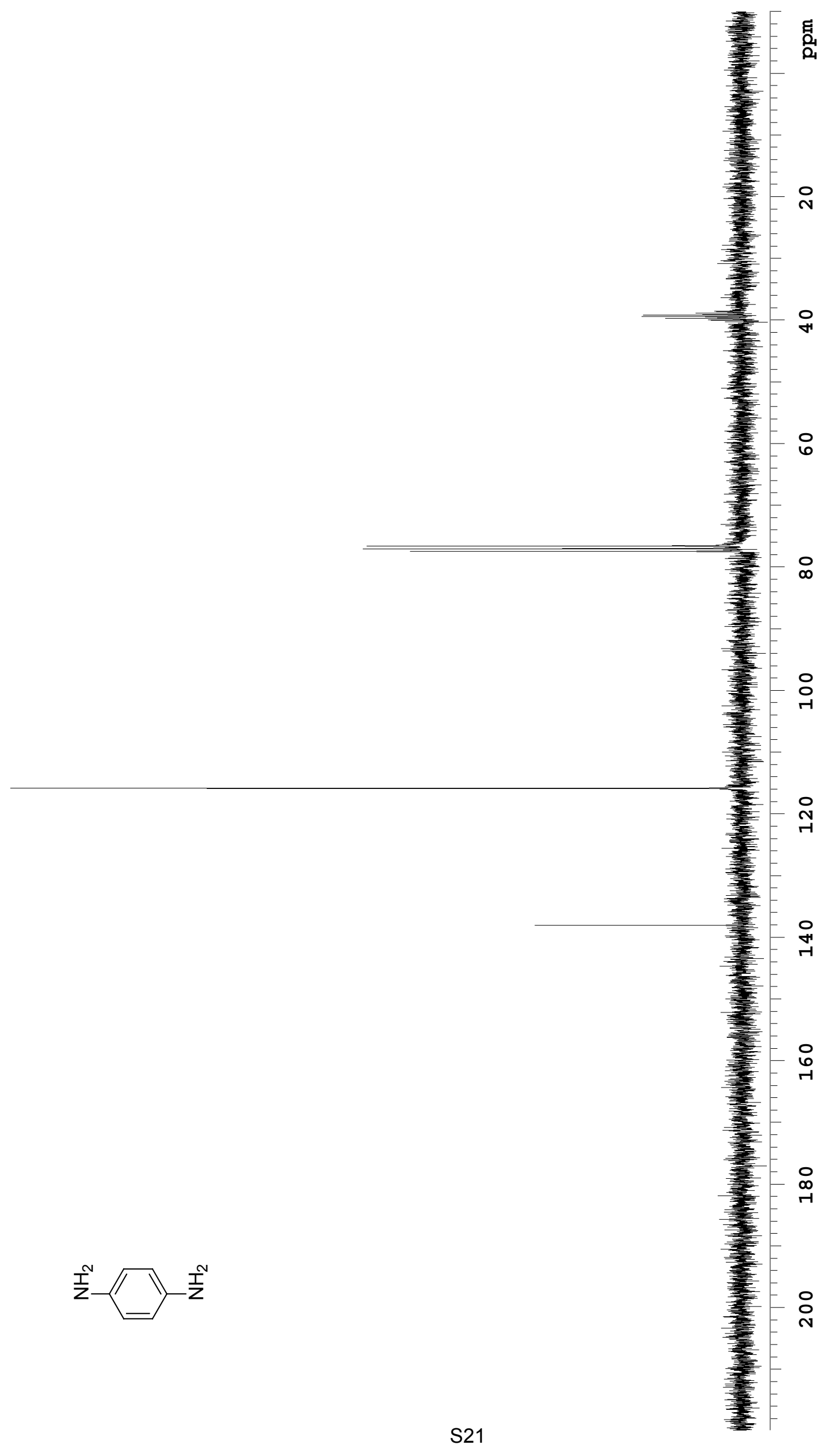




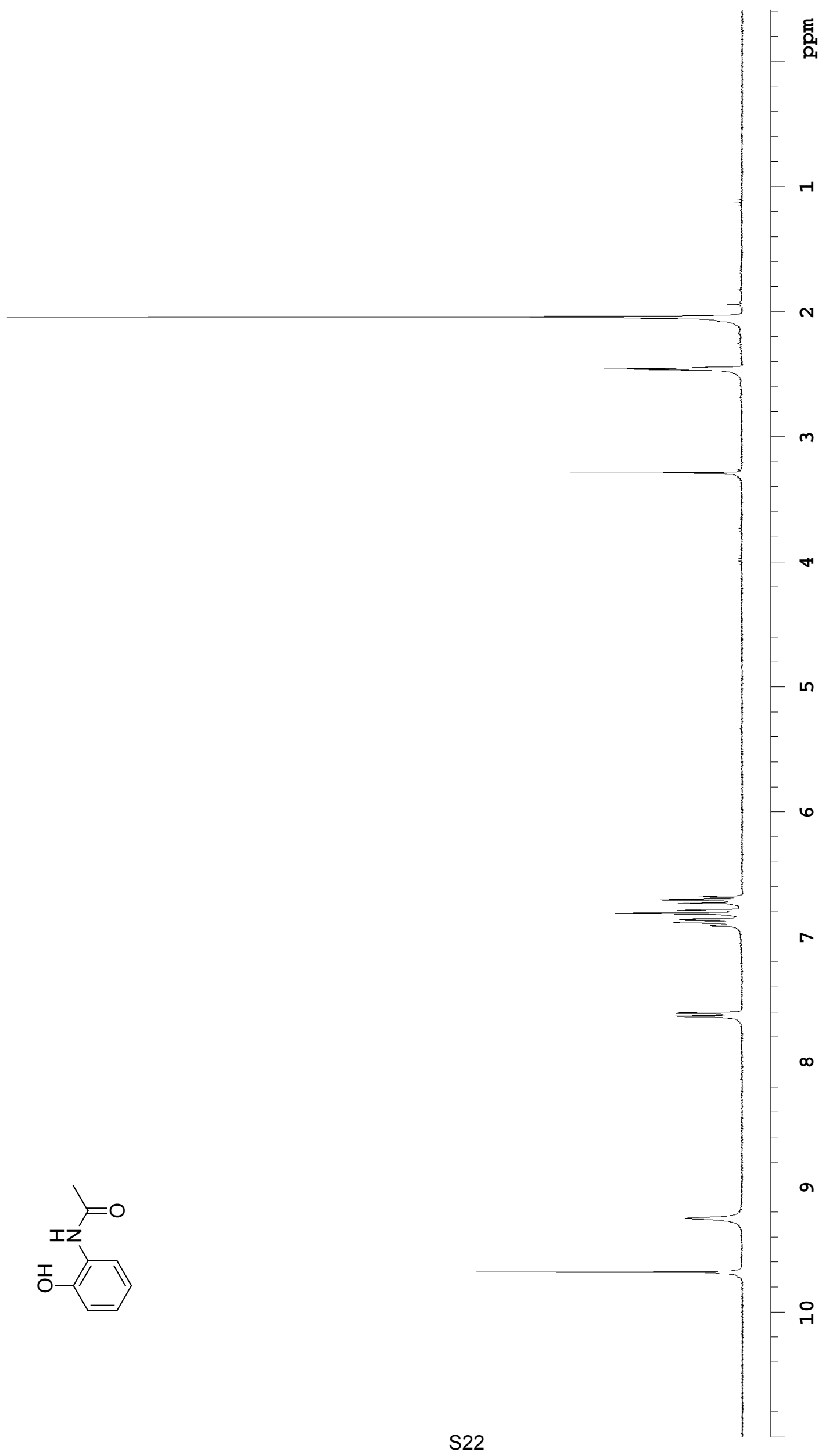




$$
1
$$




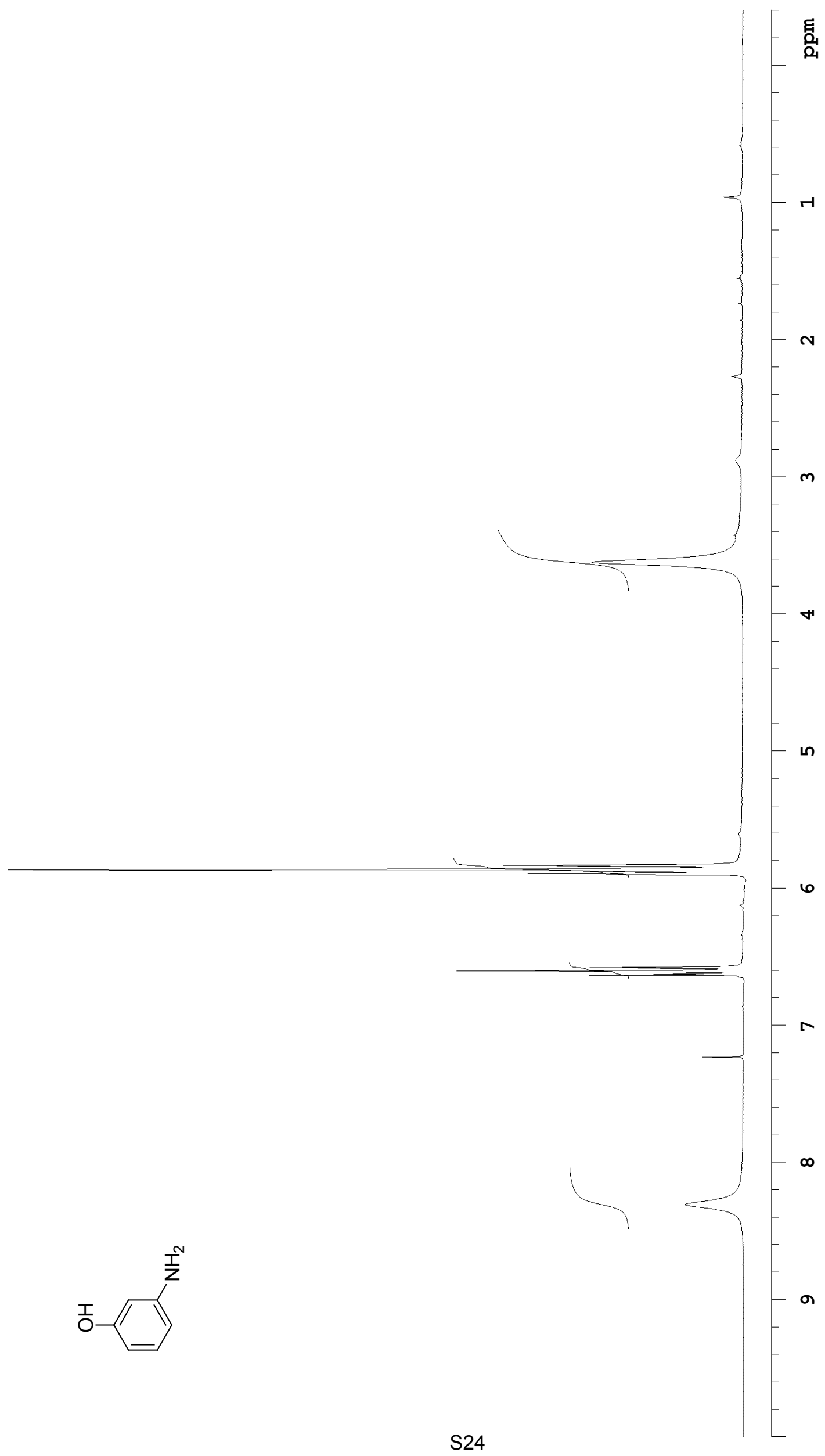




$$
1
$$




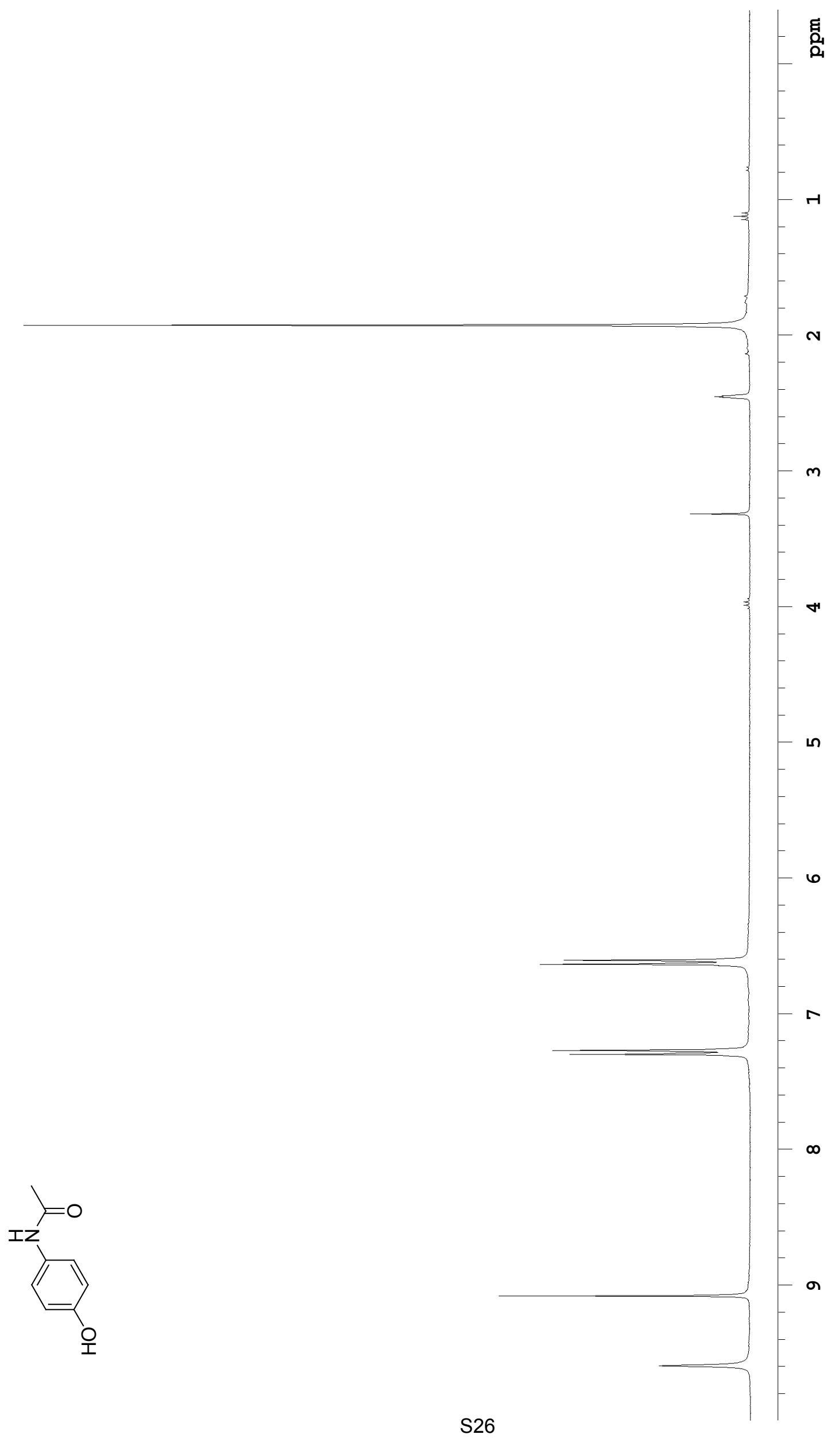




$$
1
$$




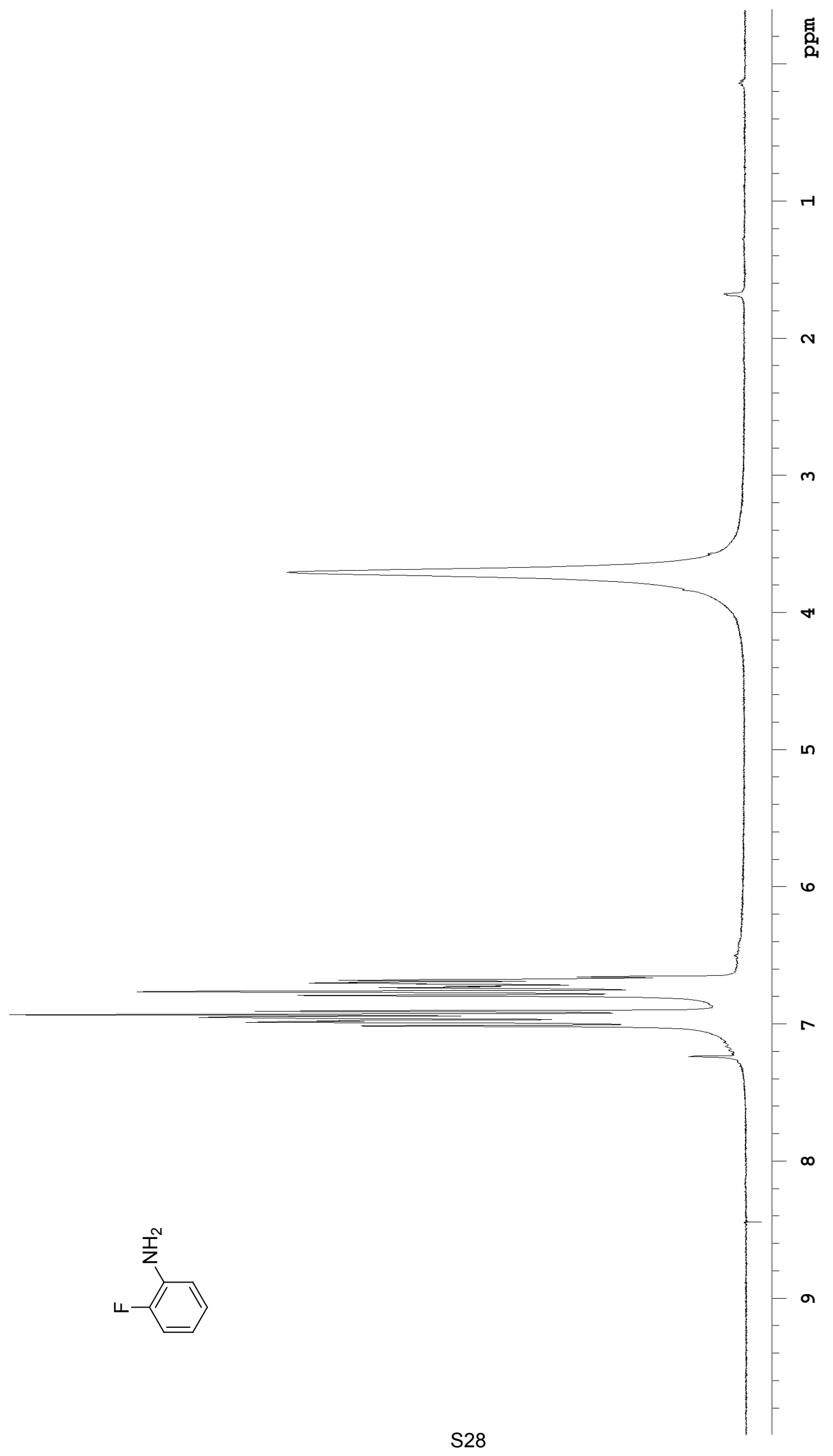




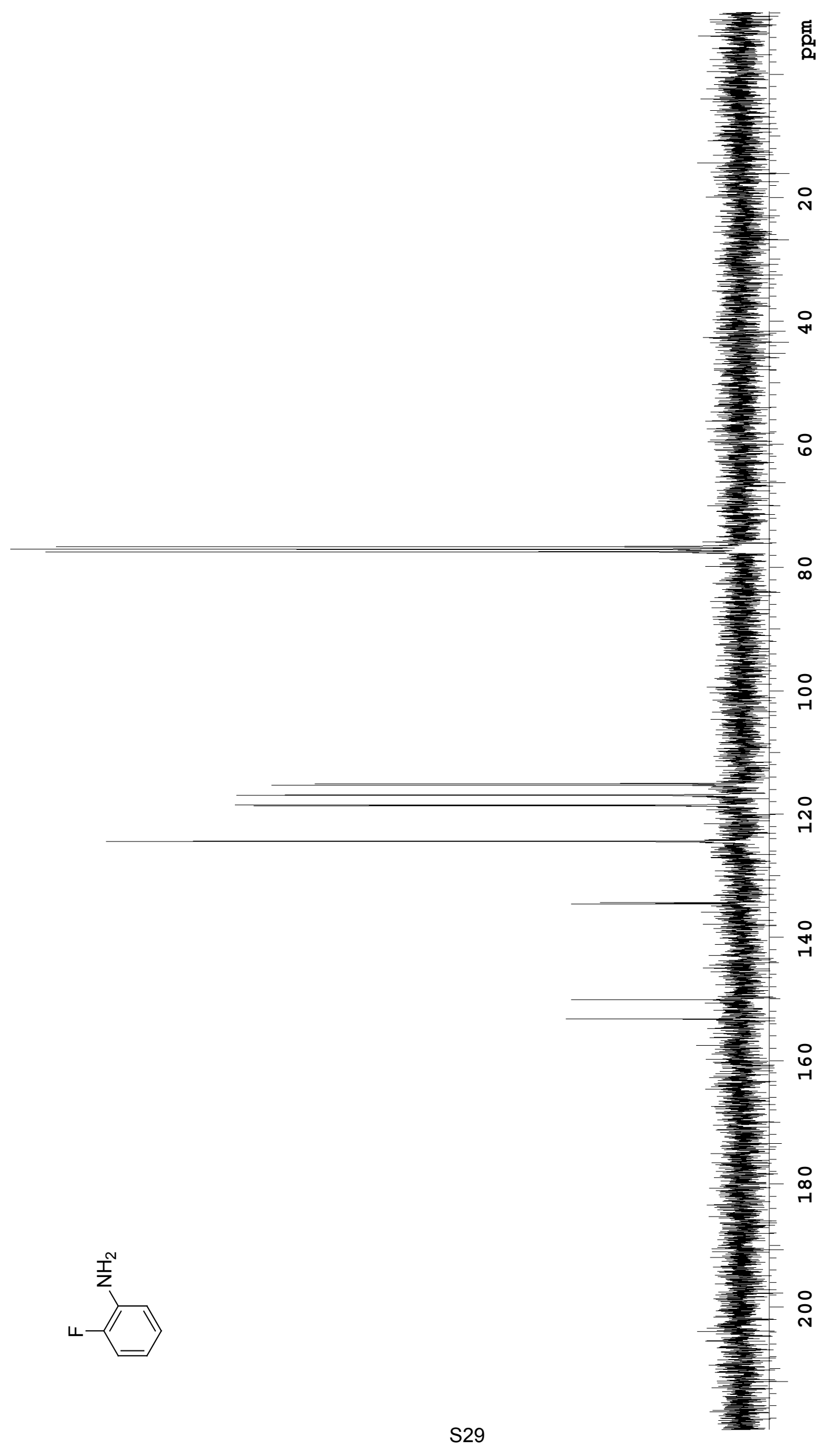




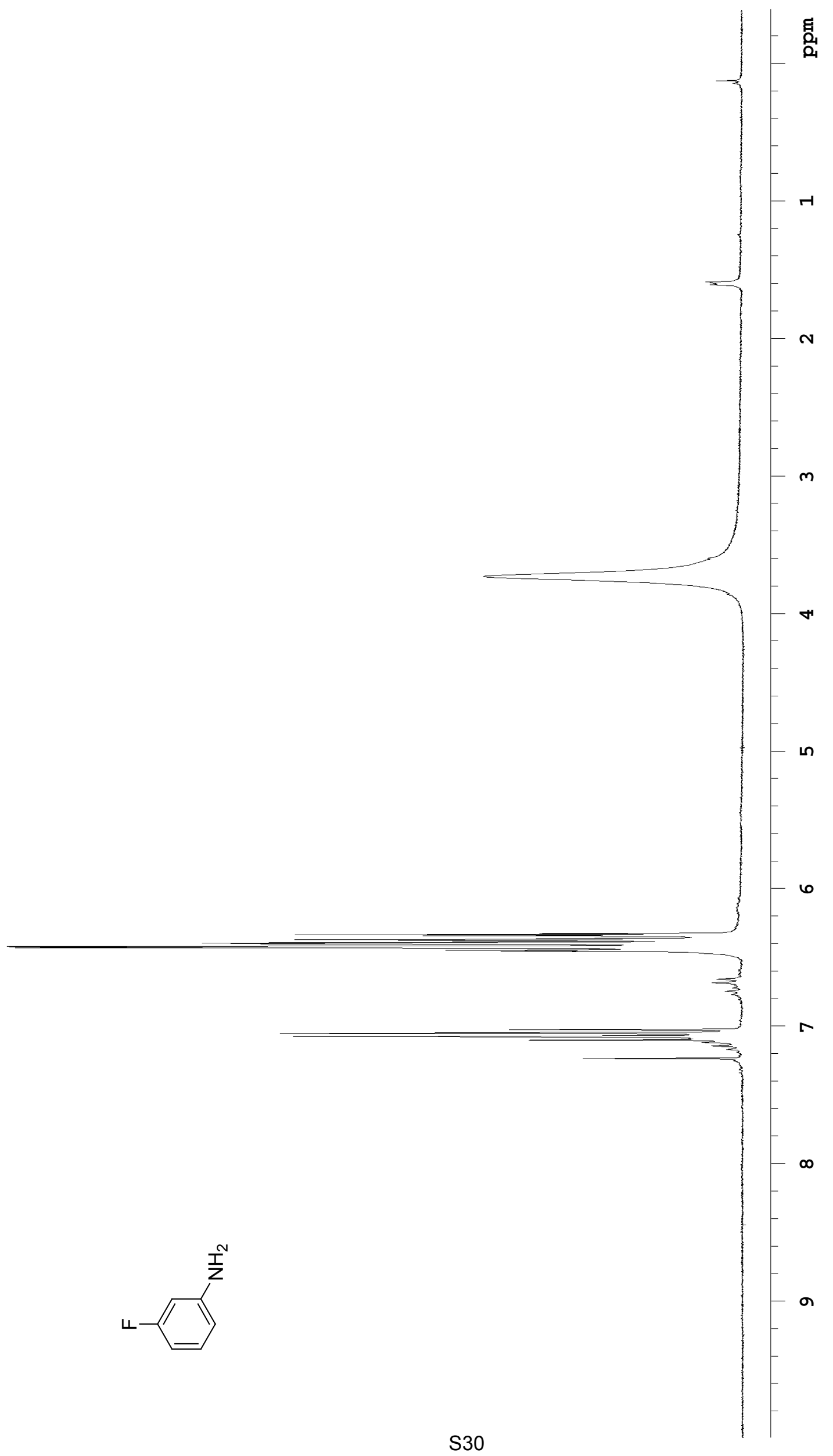




$$
\mid
$$




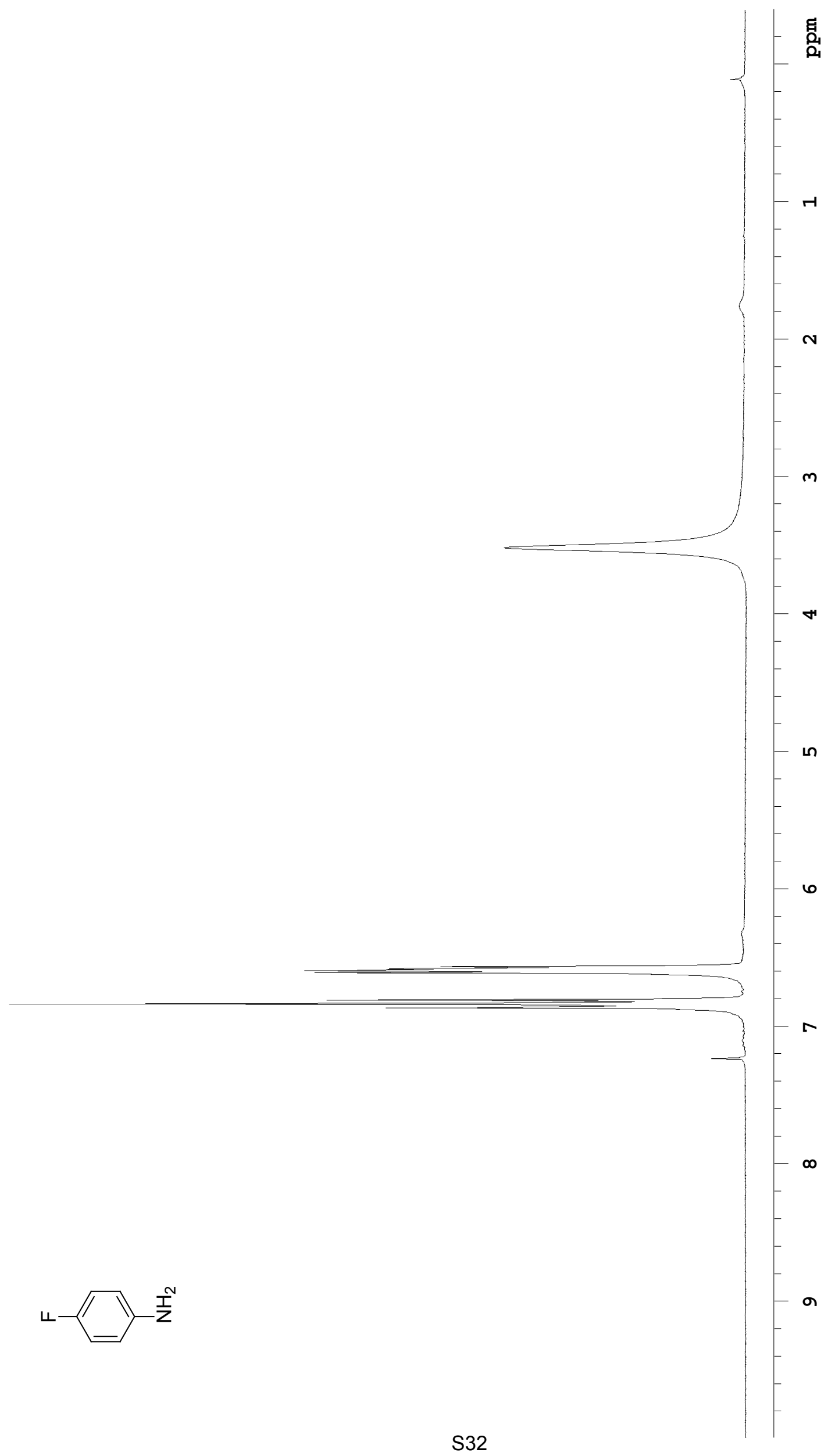




$$
1
$$




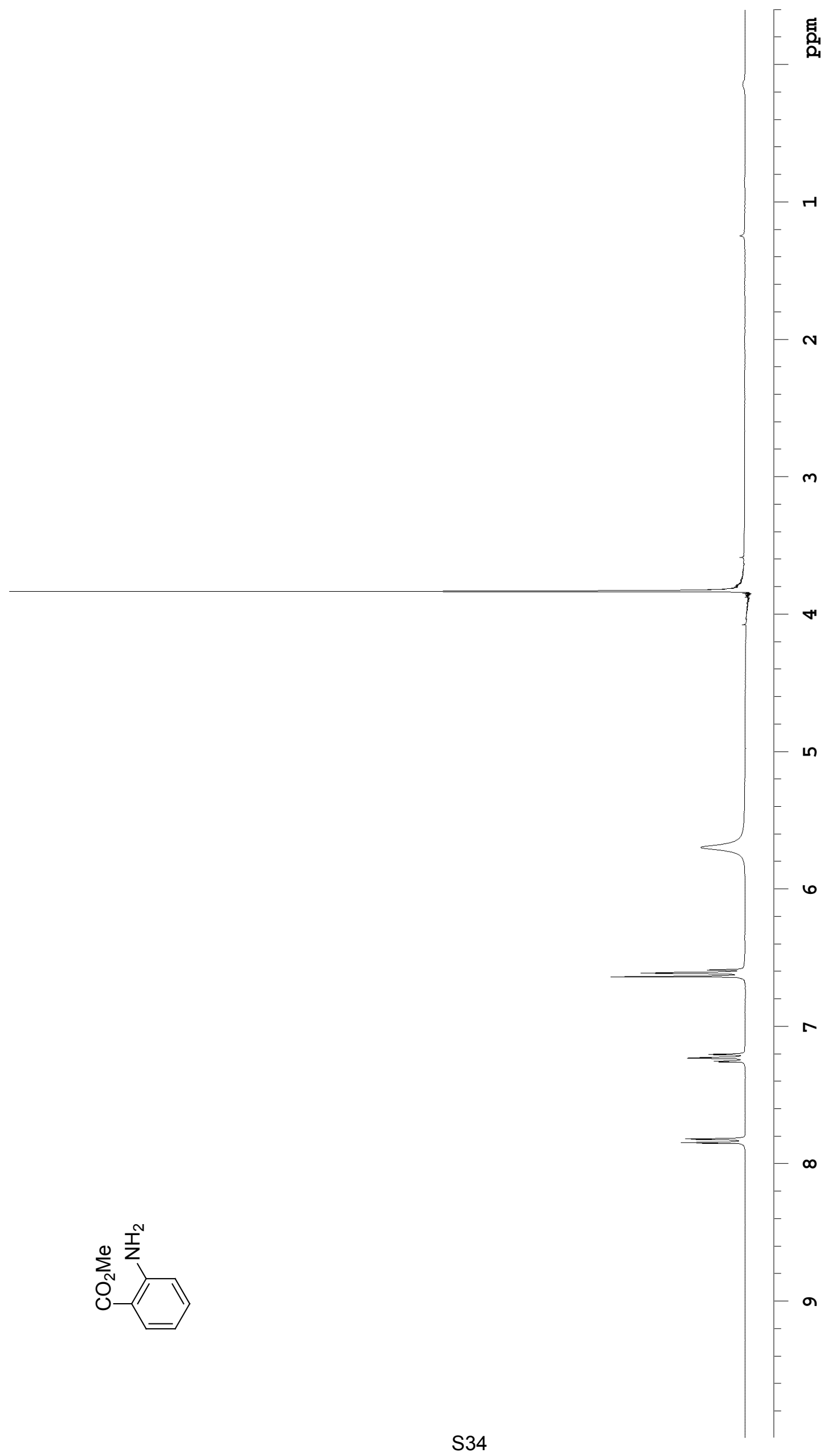




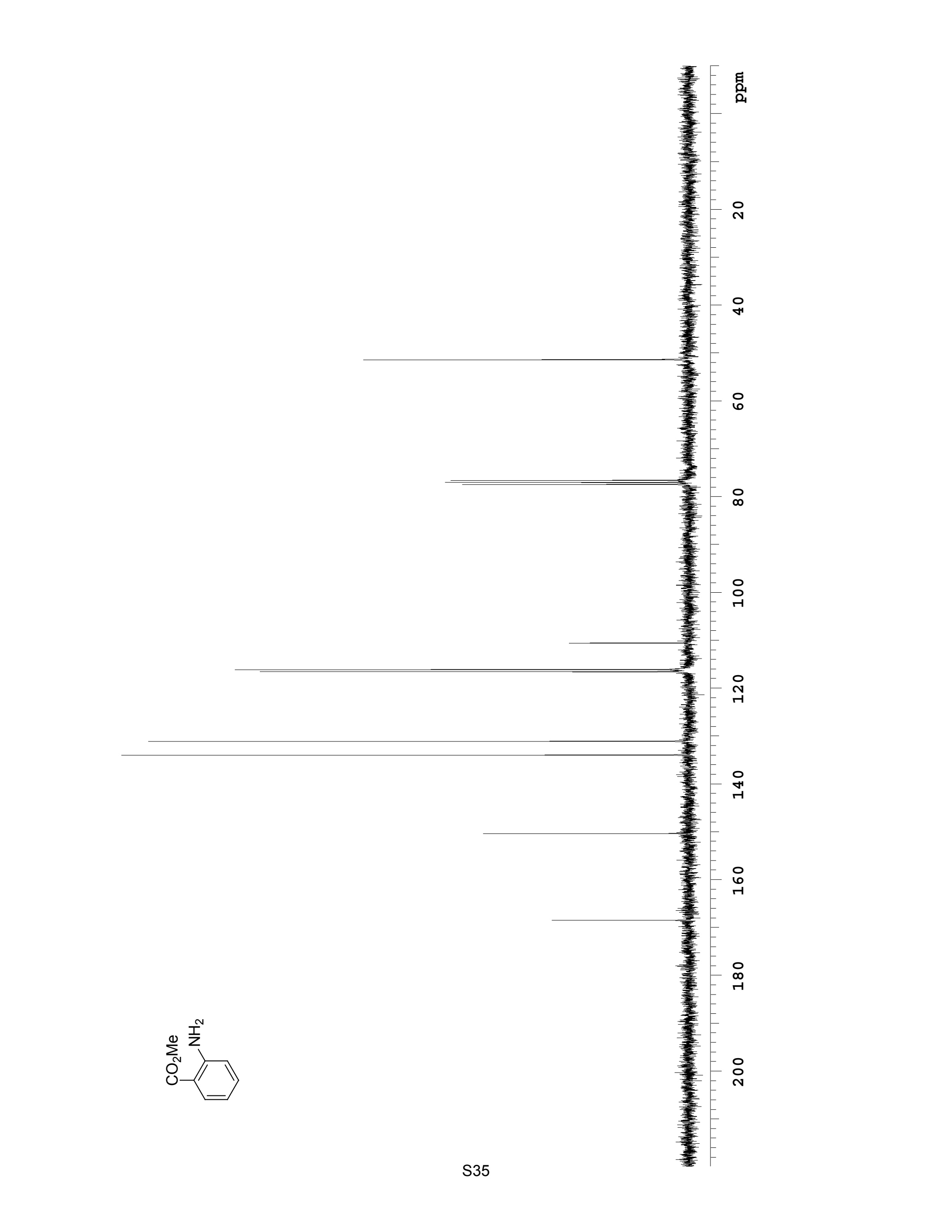




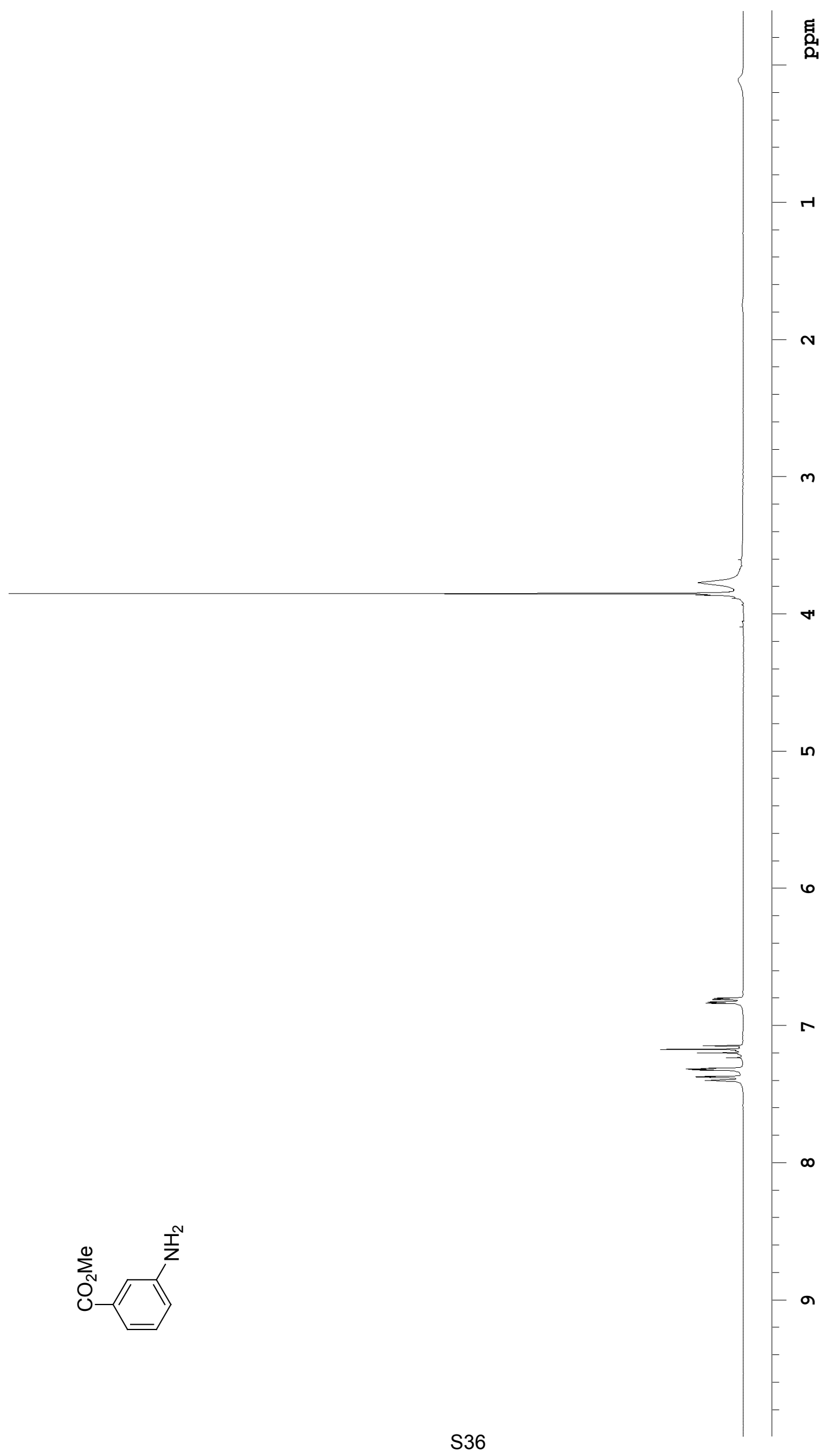




$$
\mid
$$




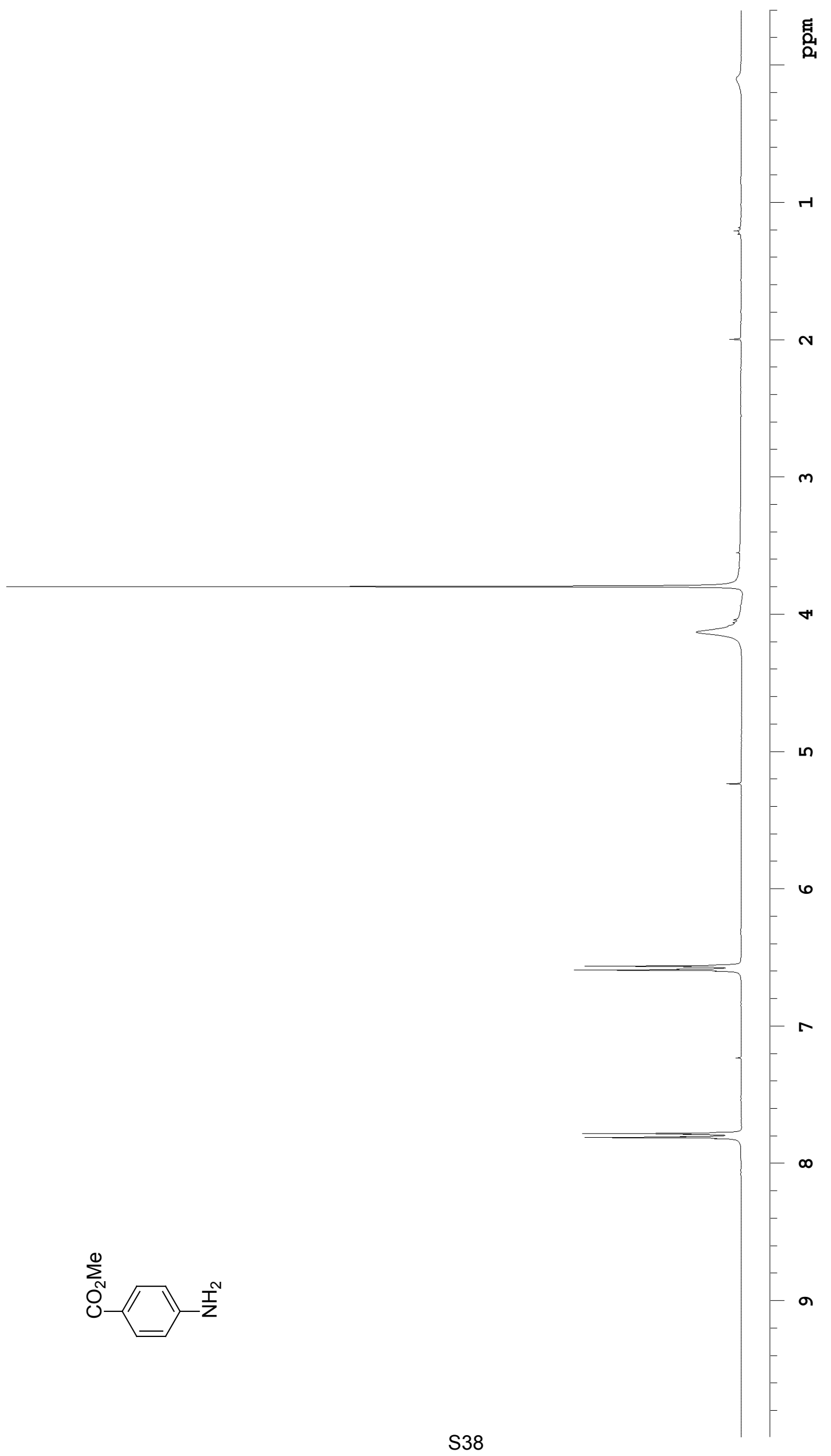




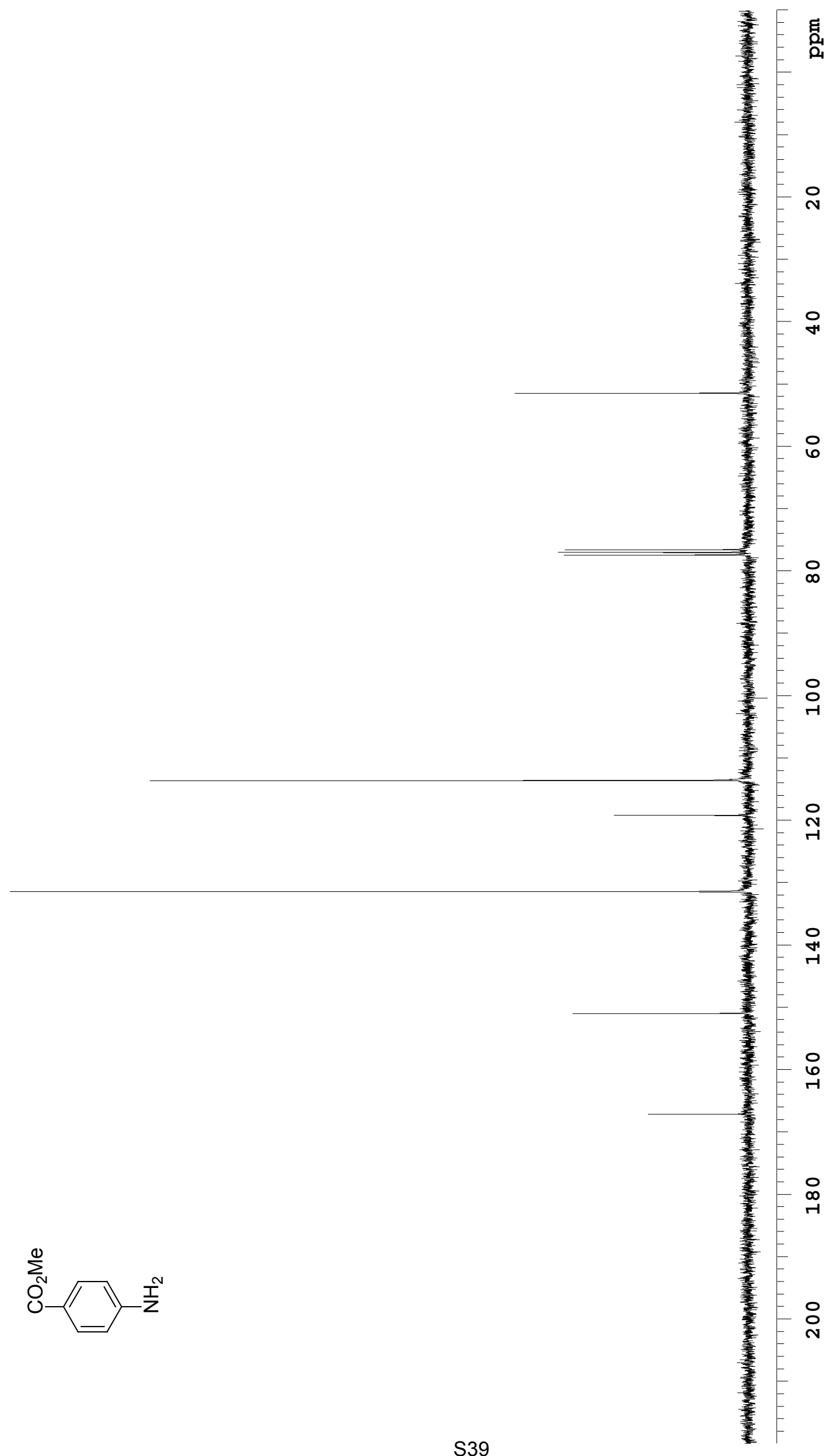




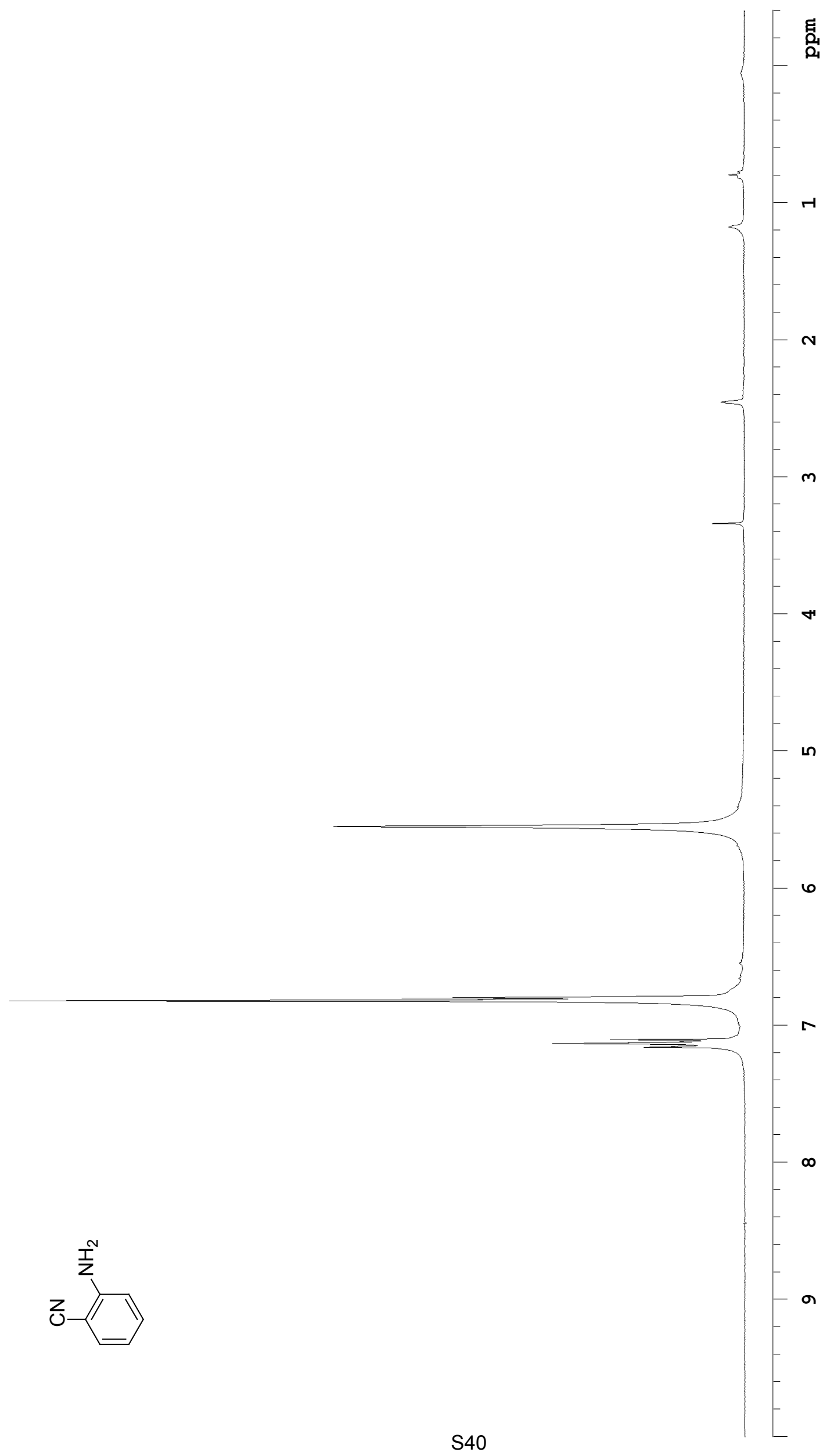




$$
1
$$




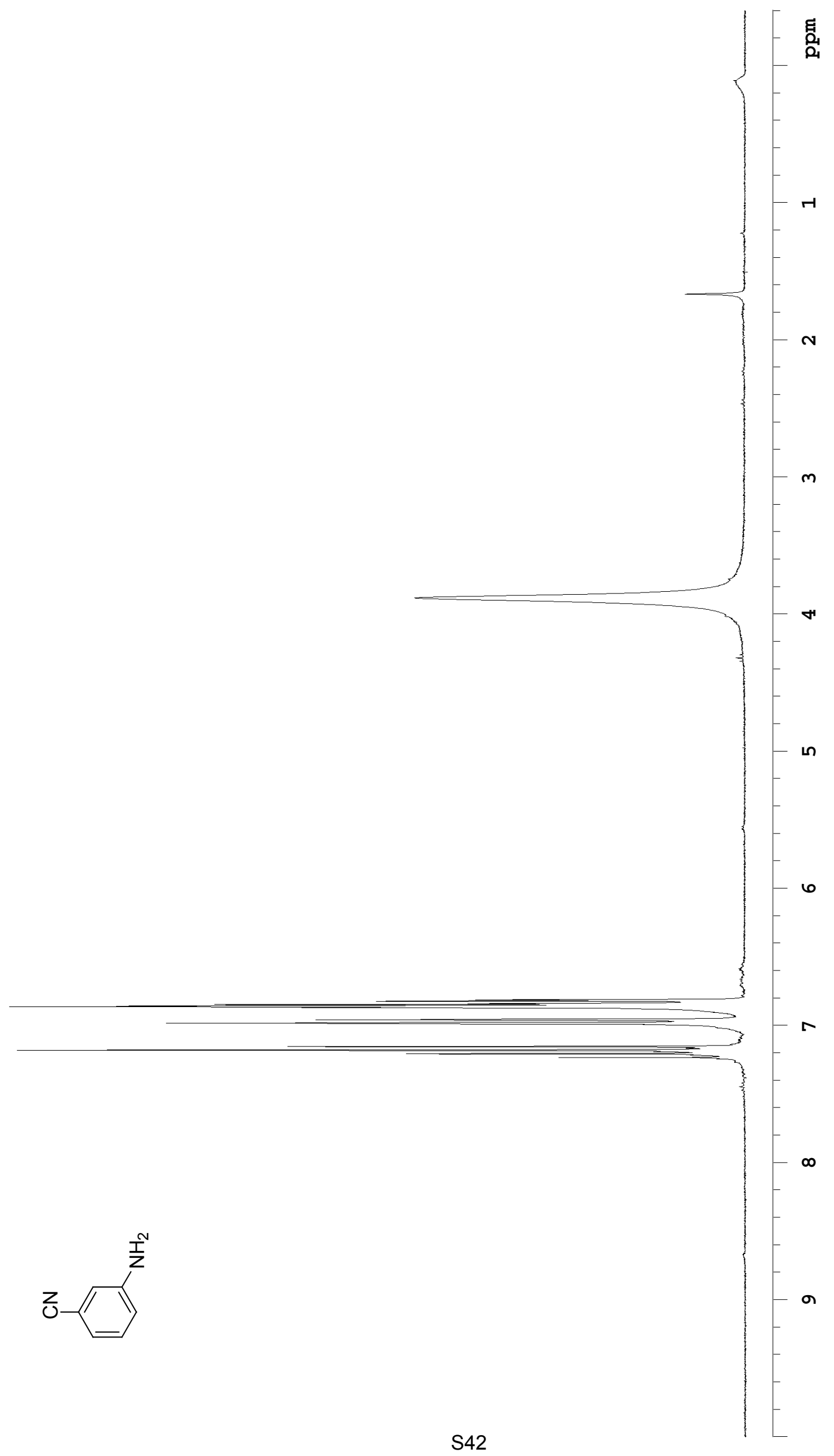




$$
1
$$




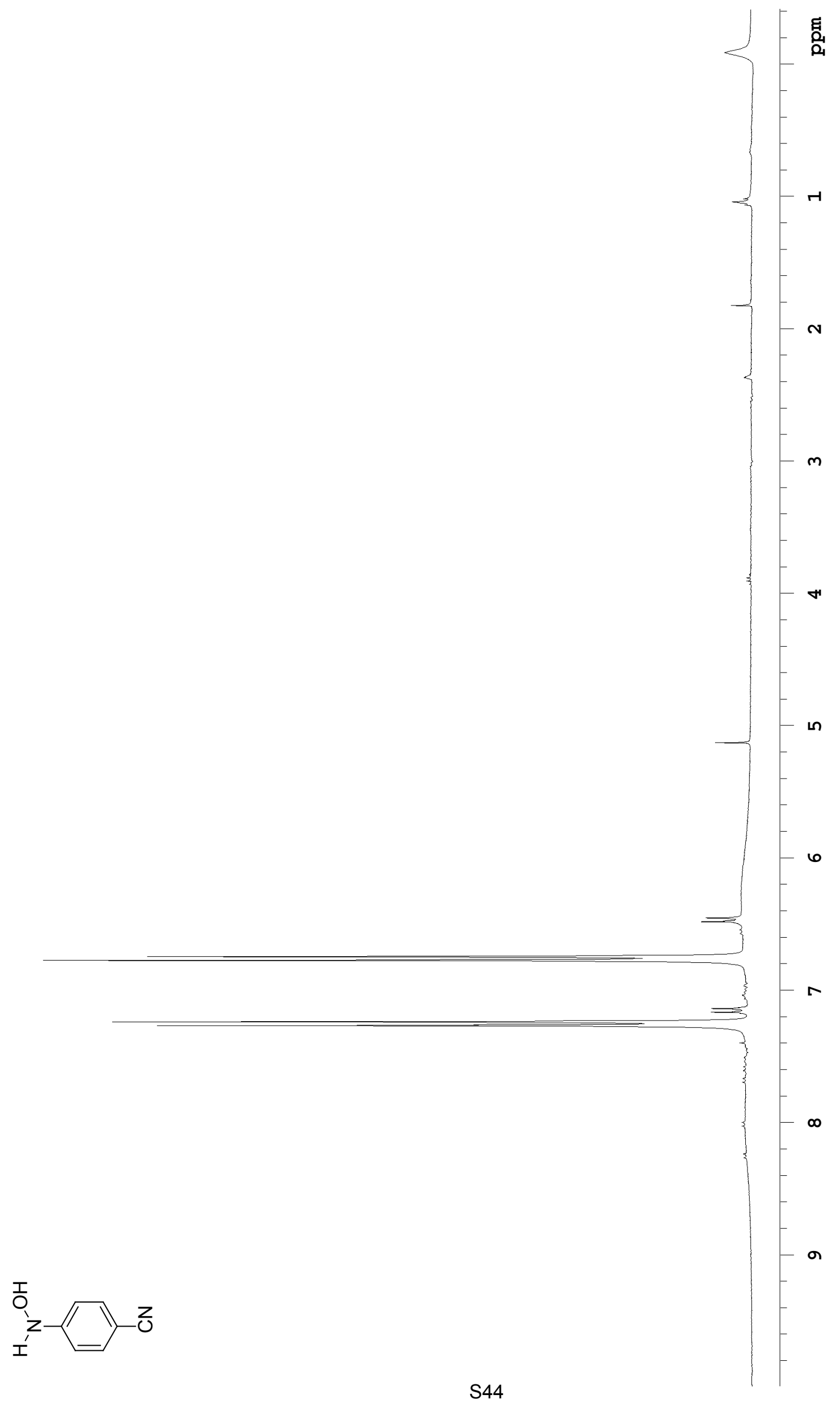




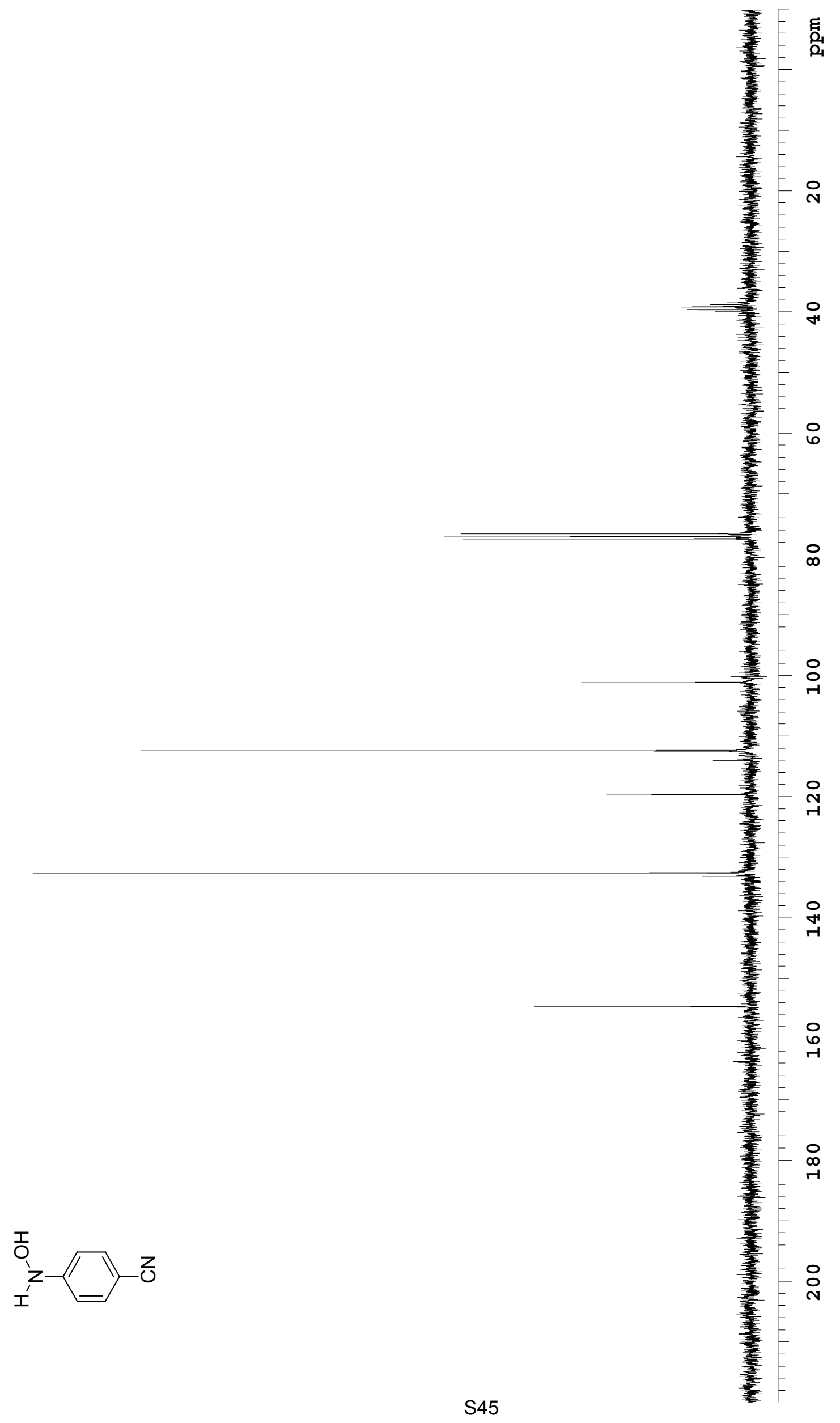




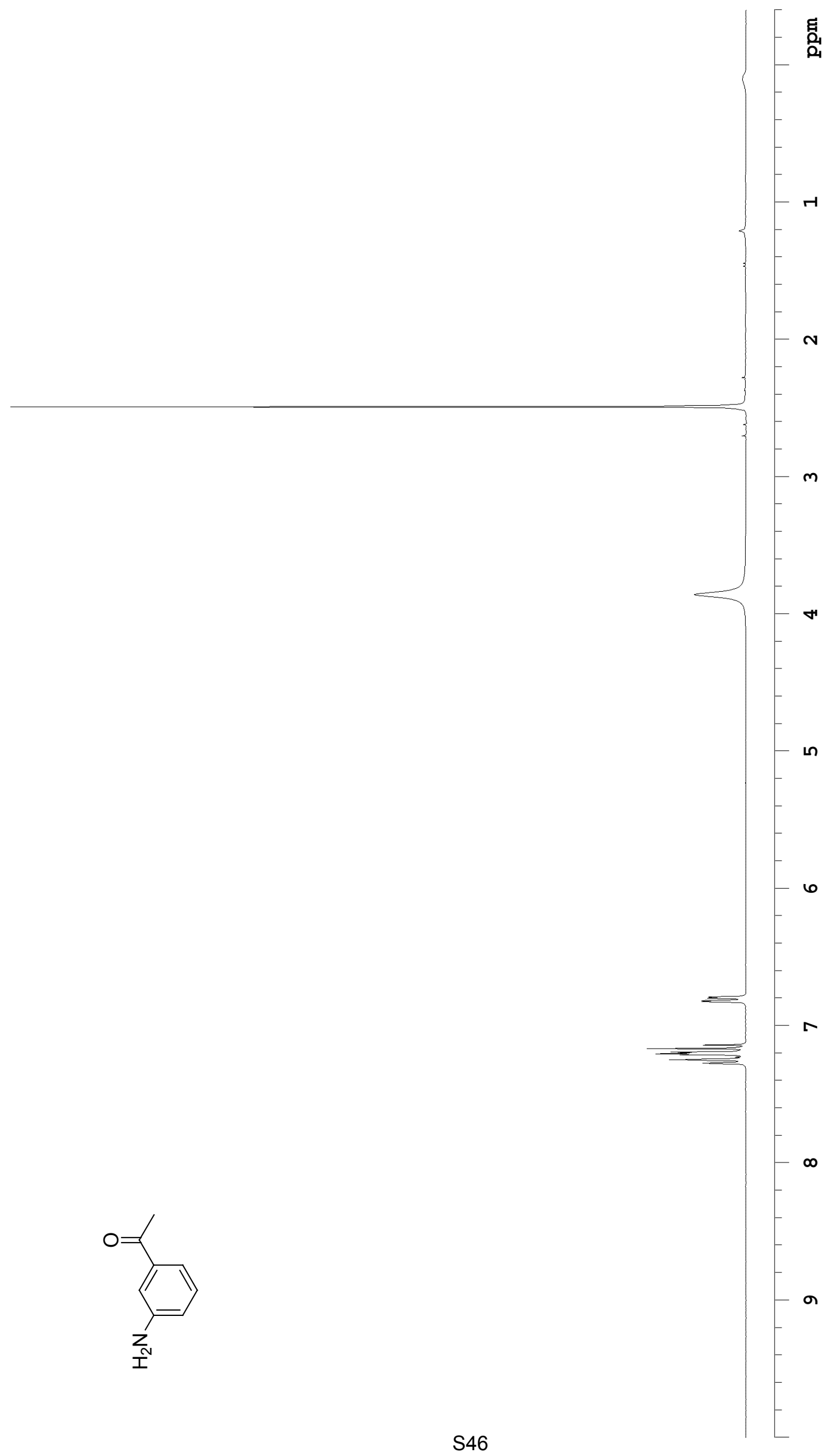




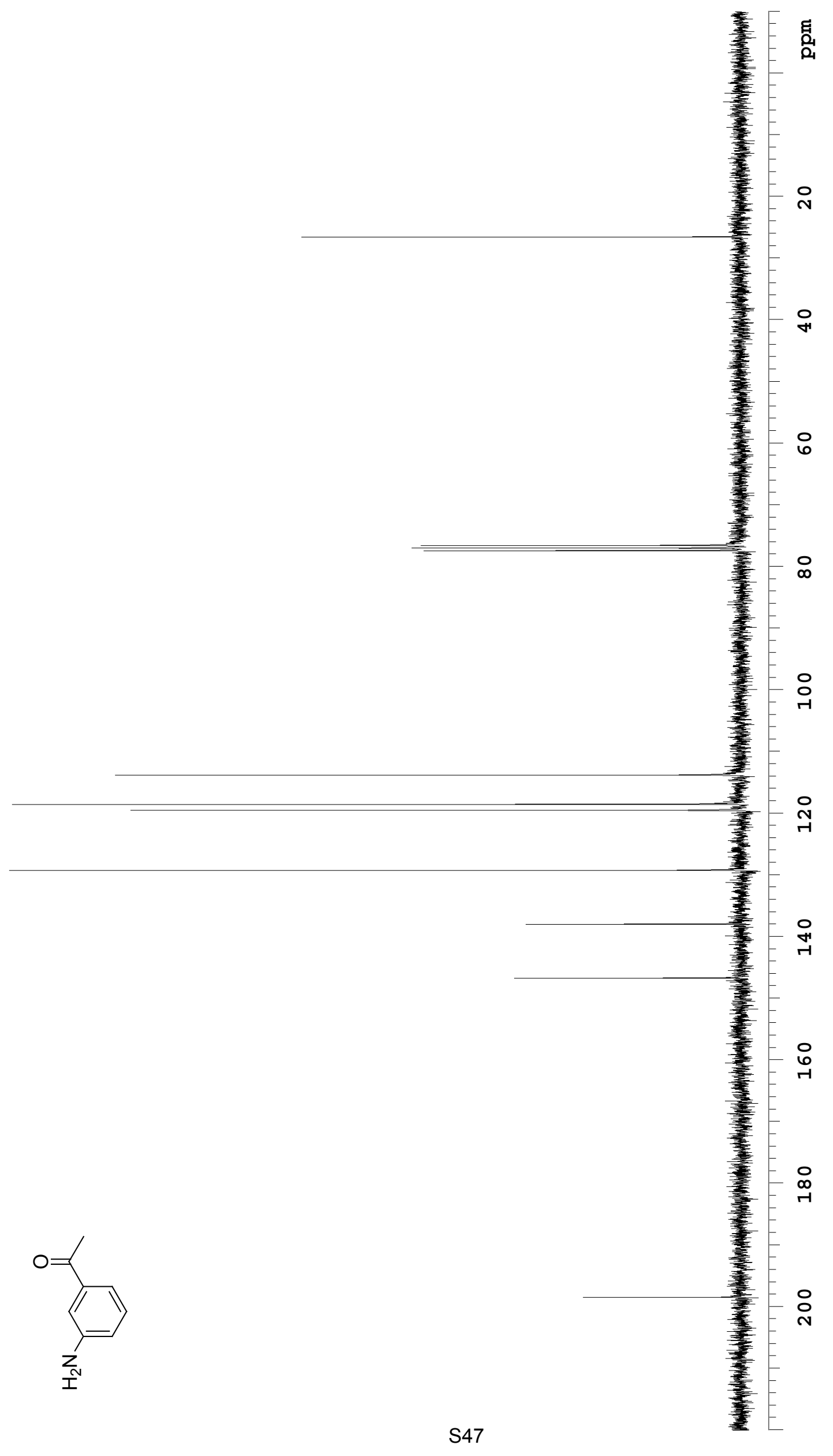




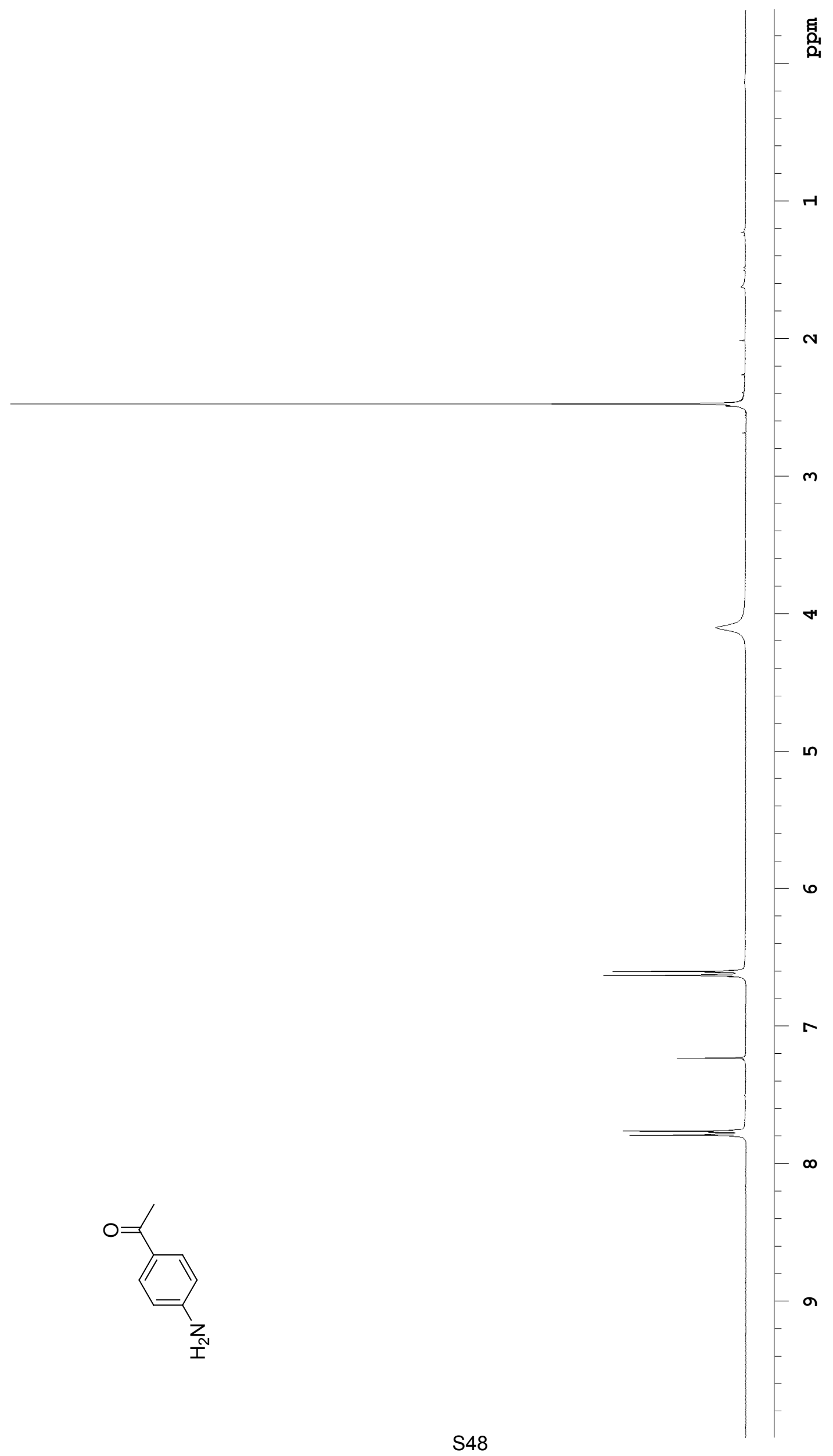




$$
1
$$




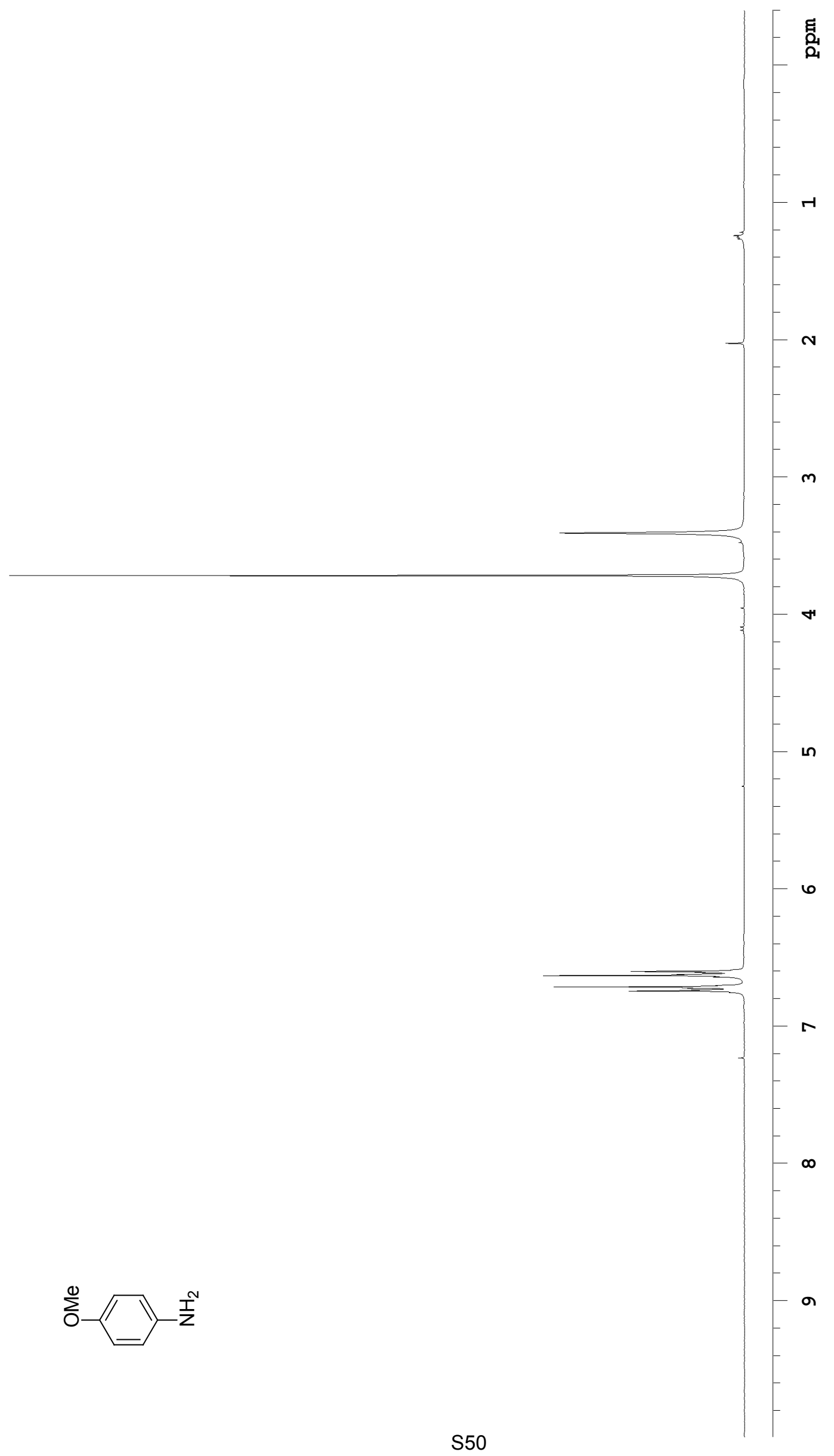




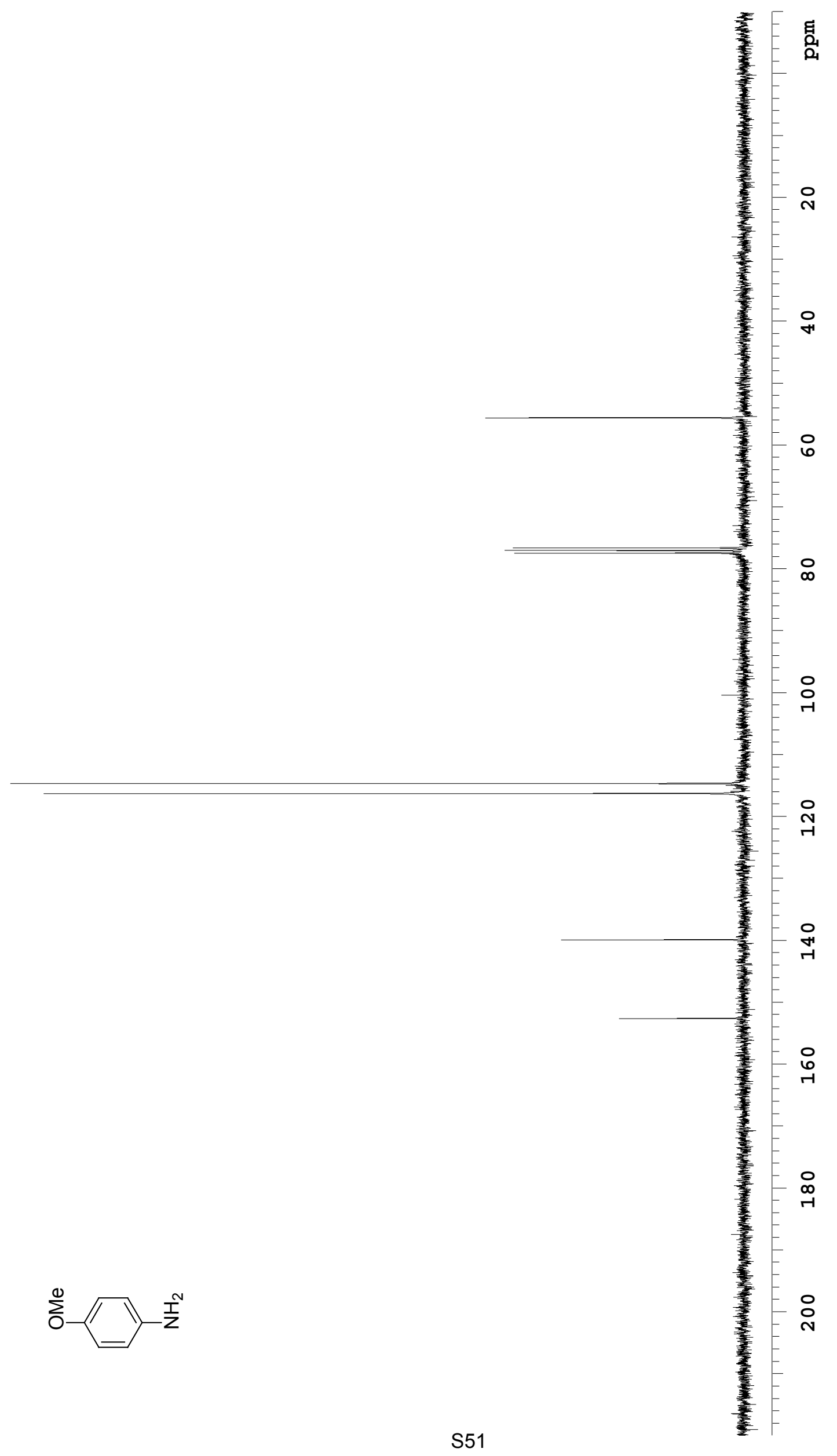




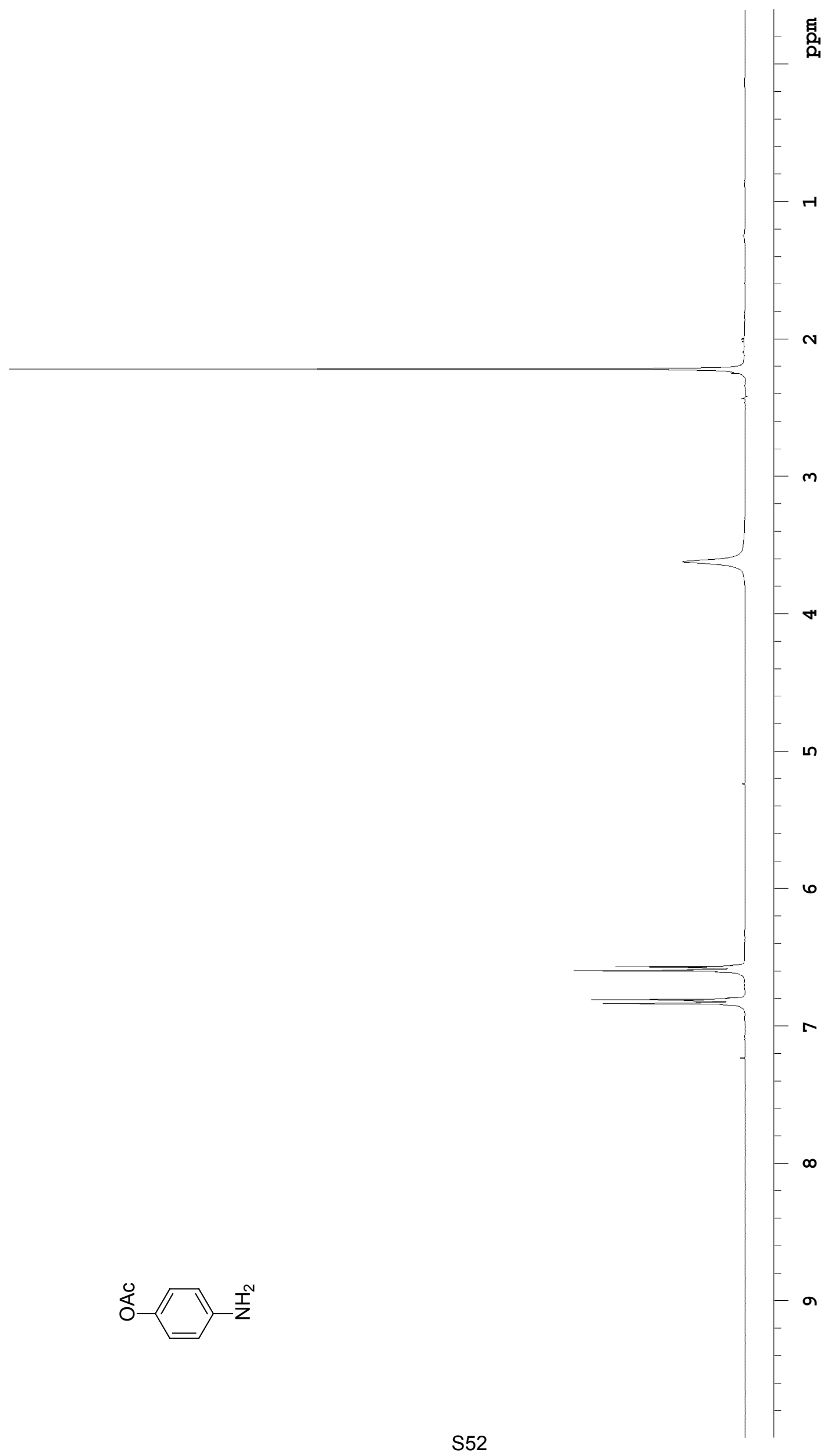




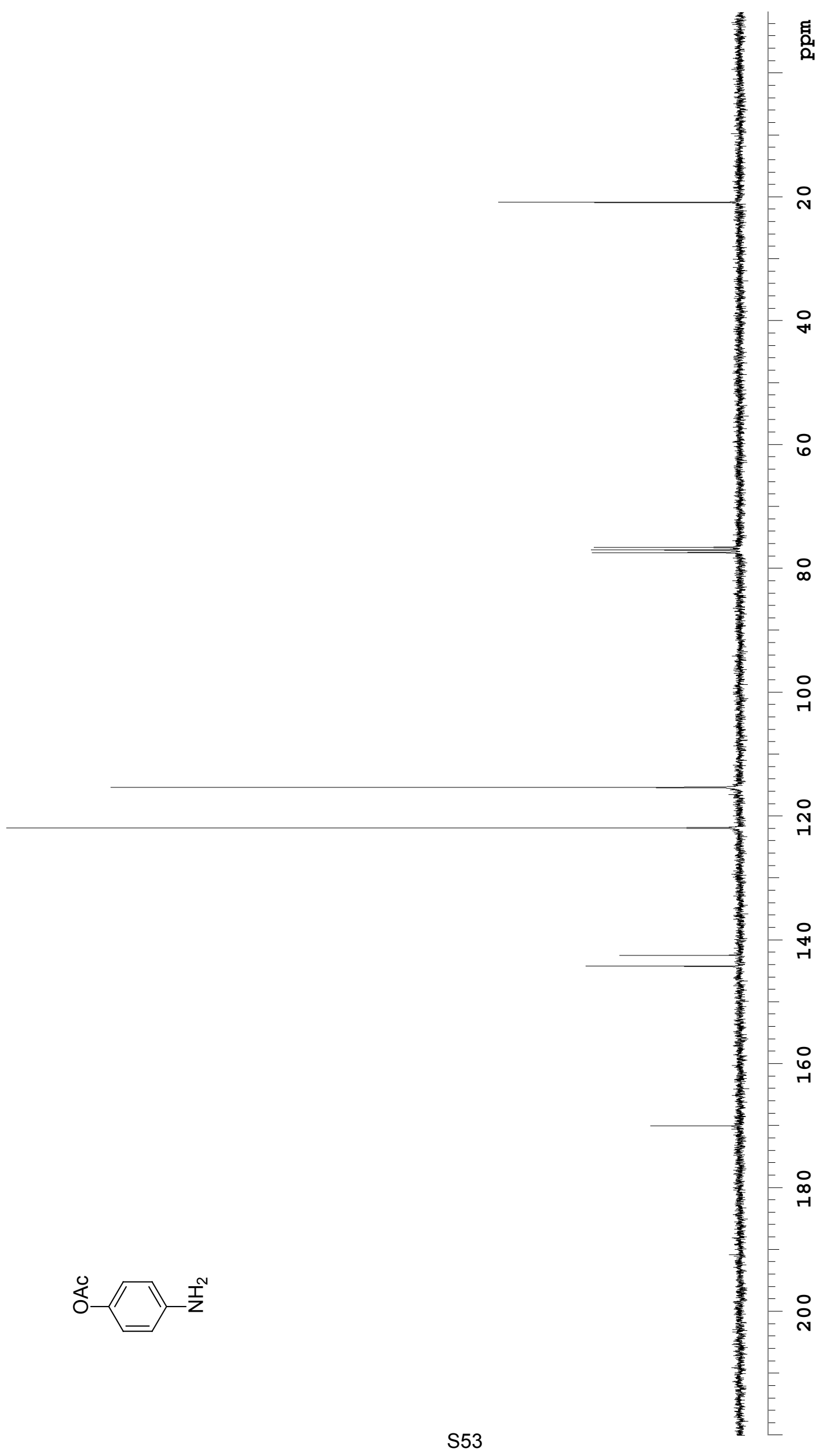




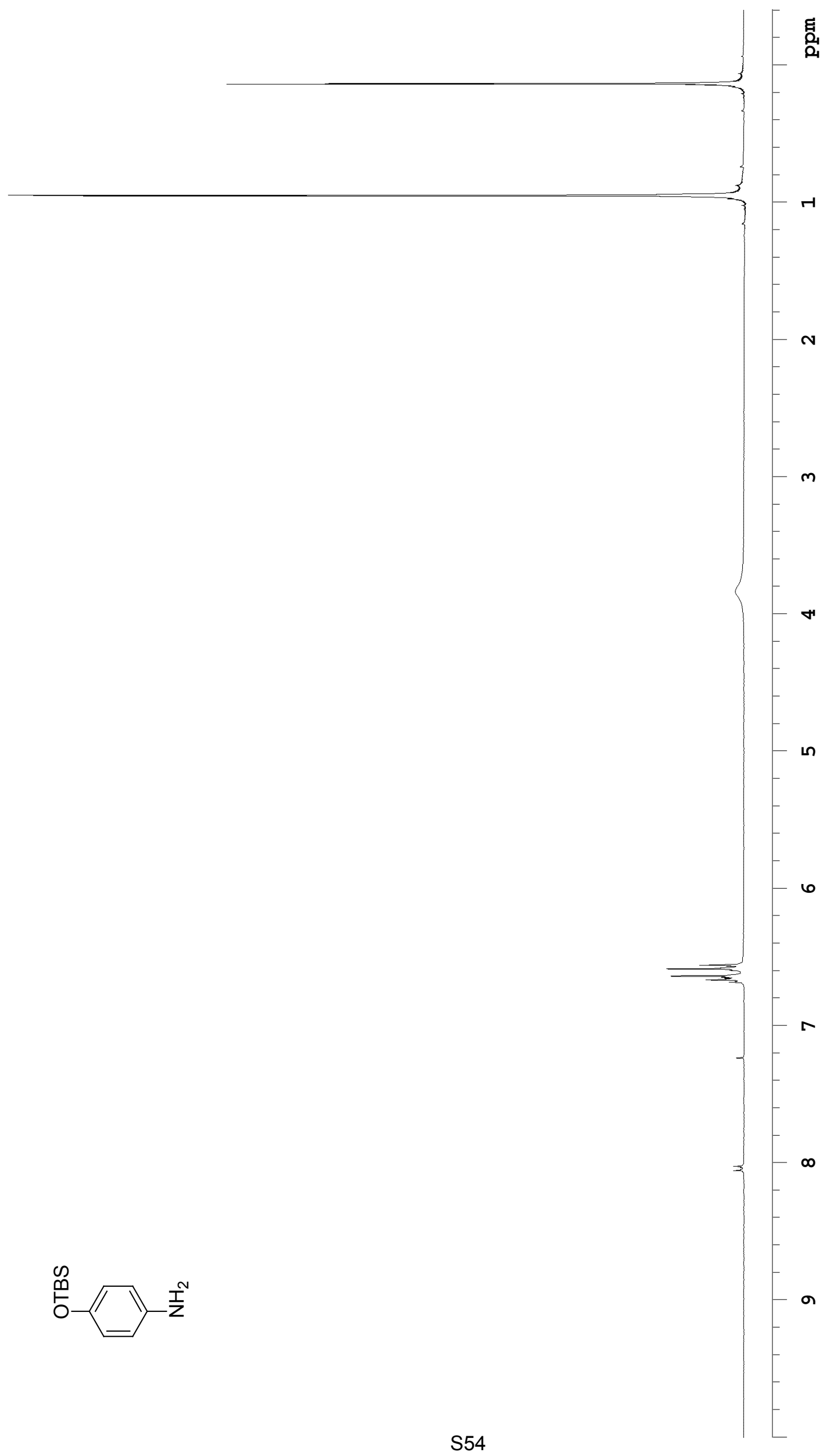




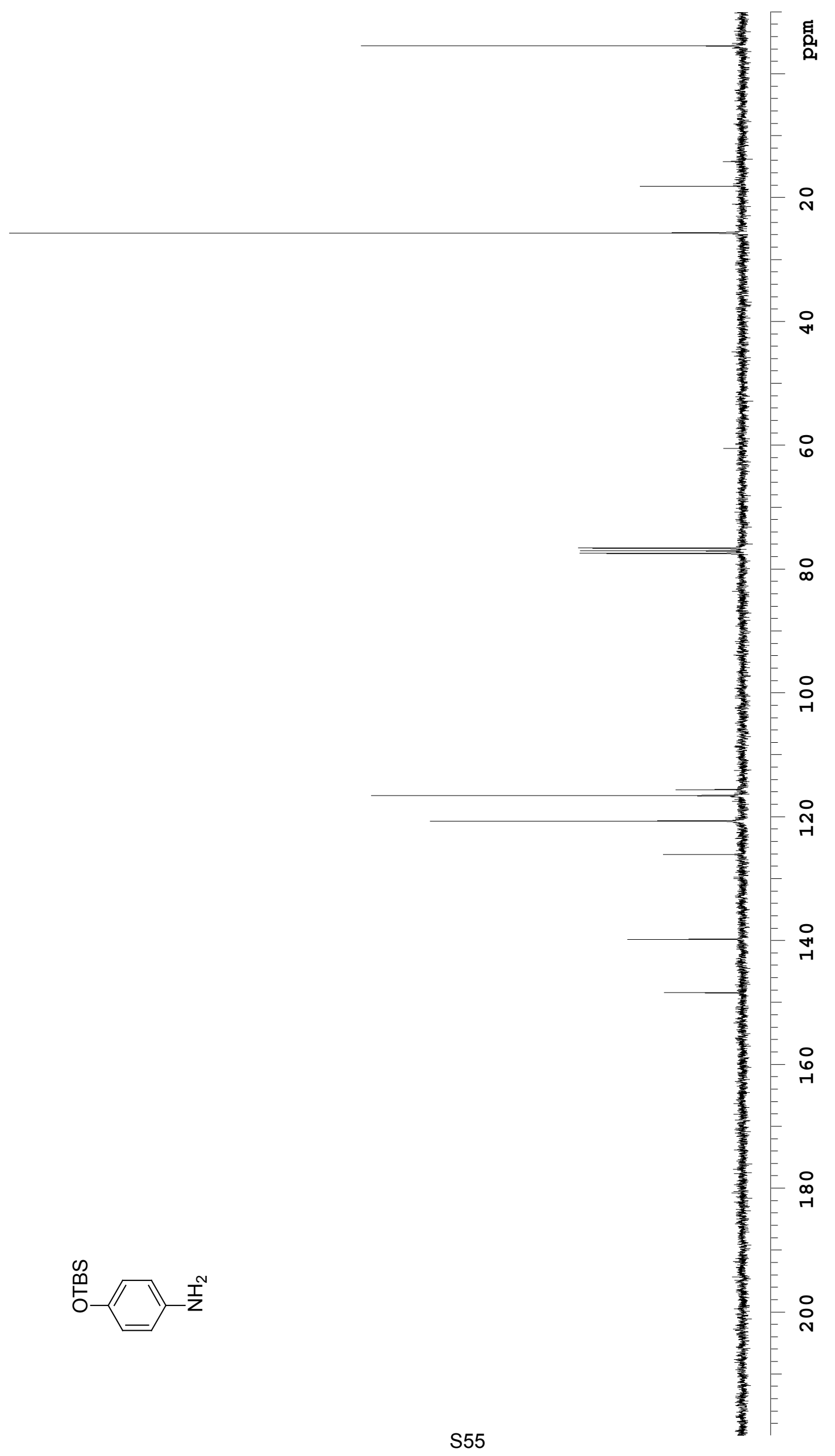




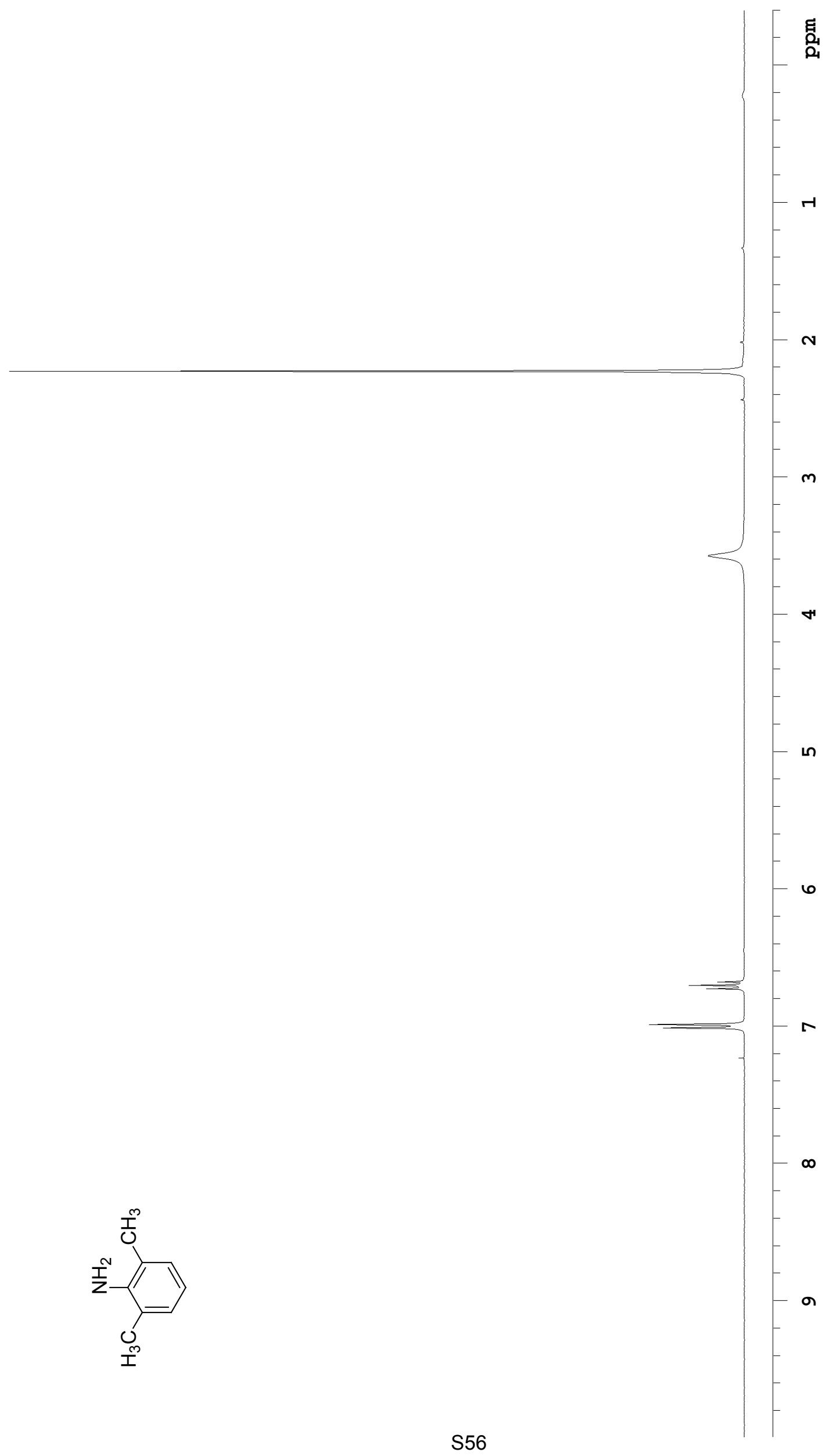




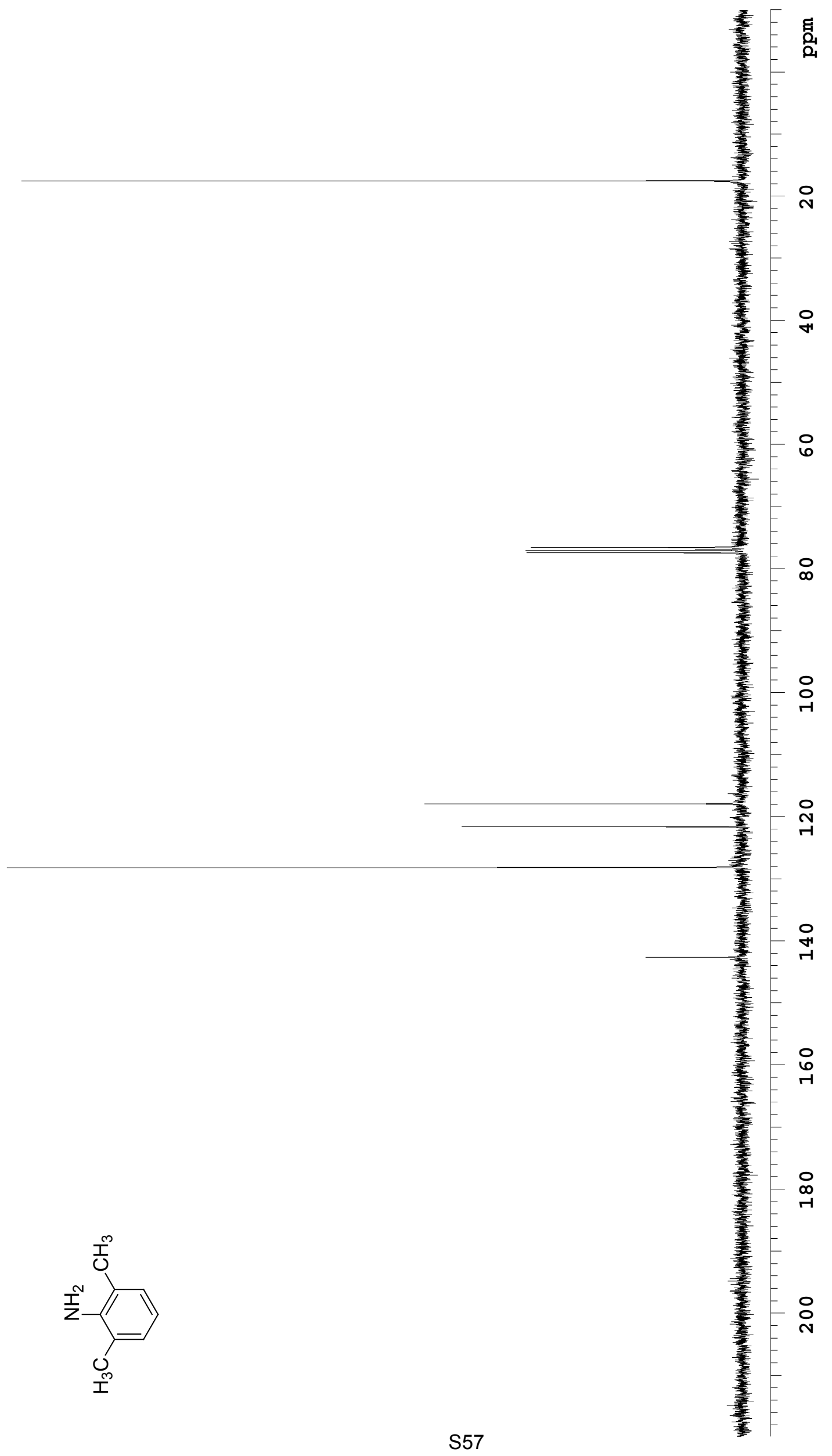



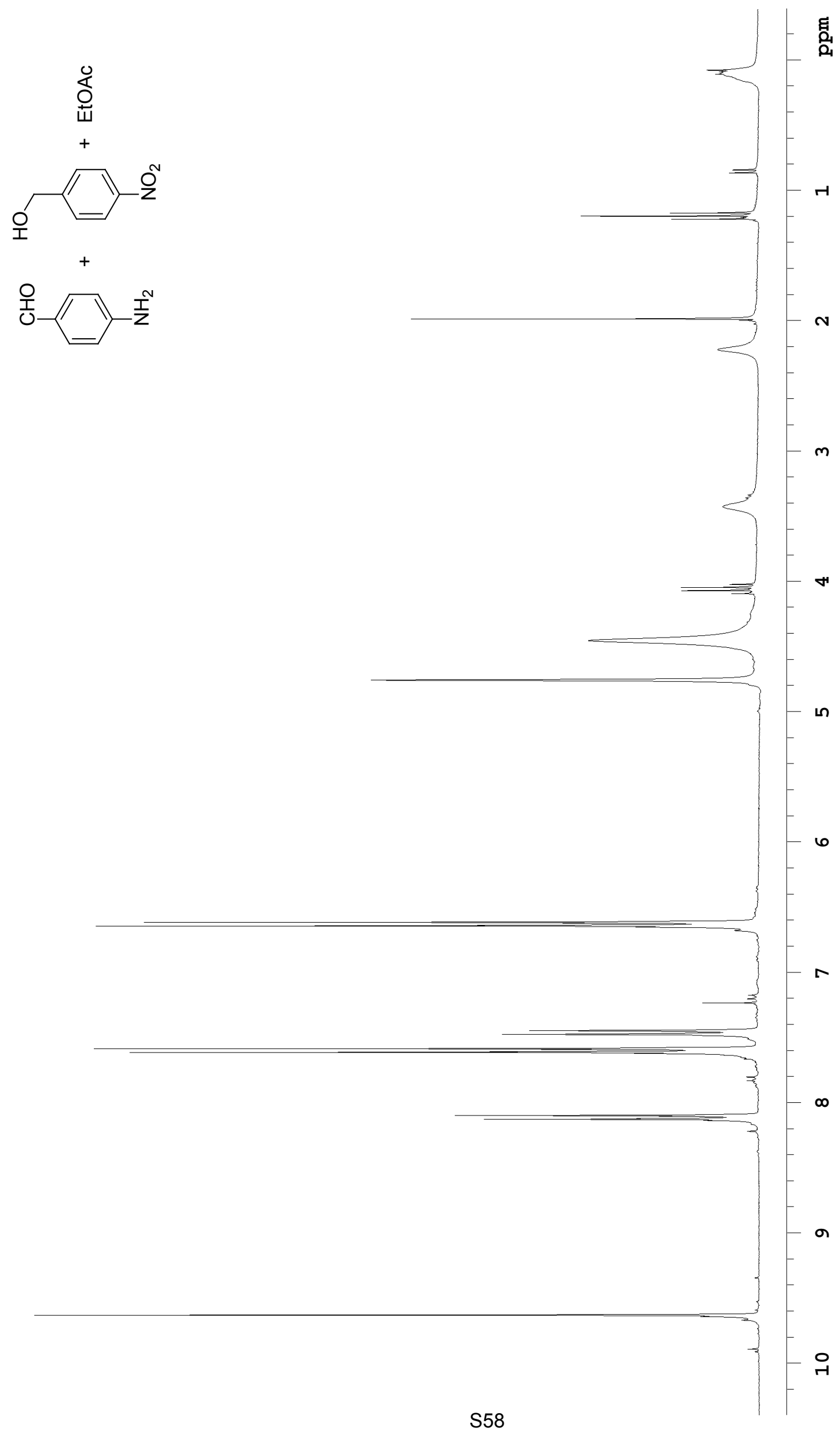


$$
1
$$




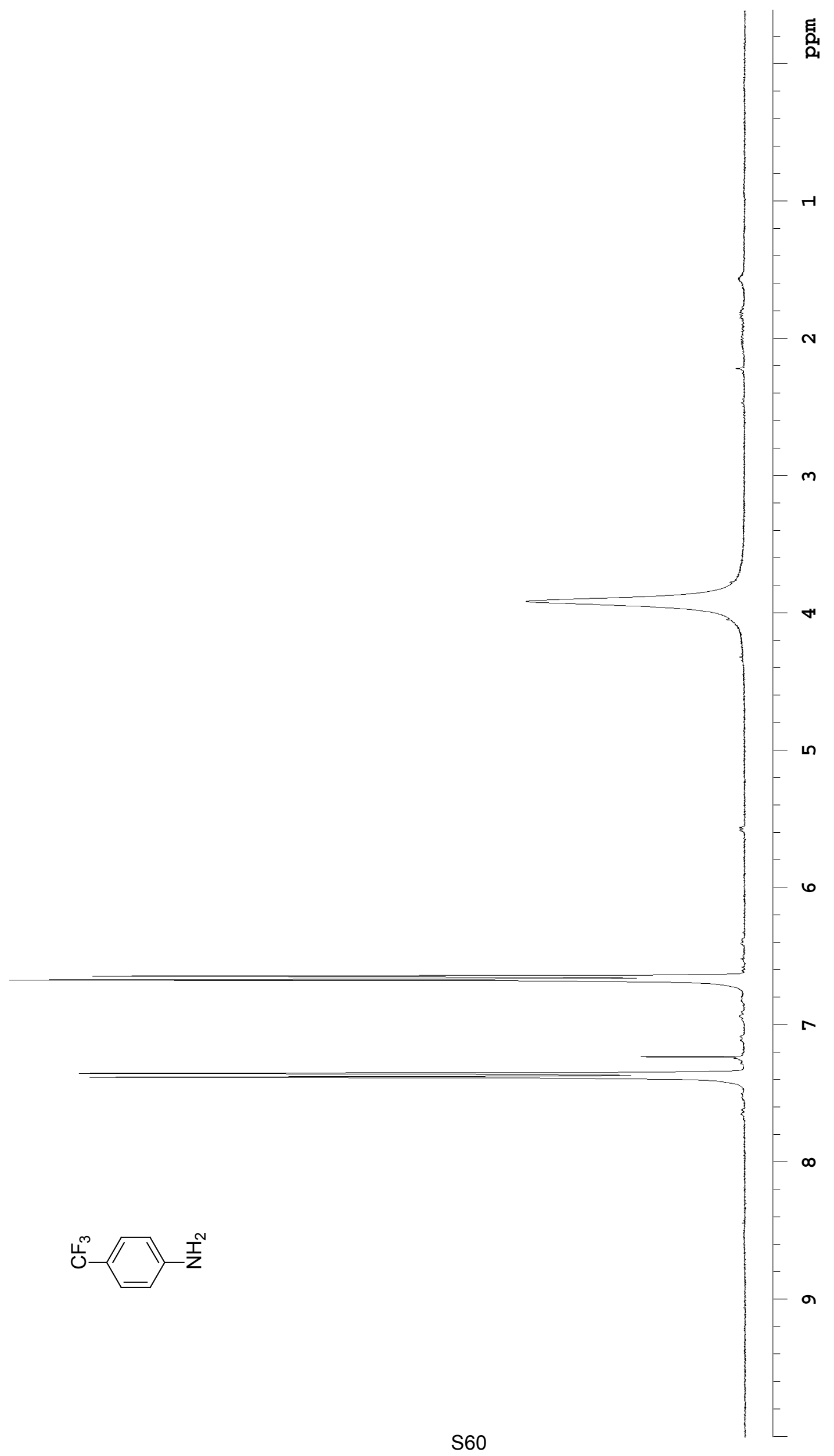




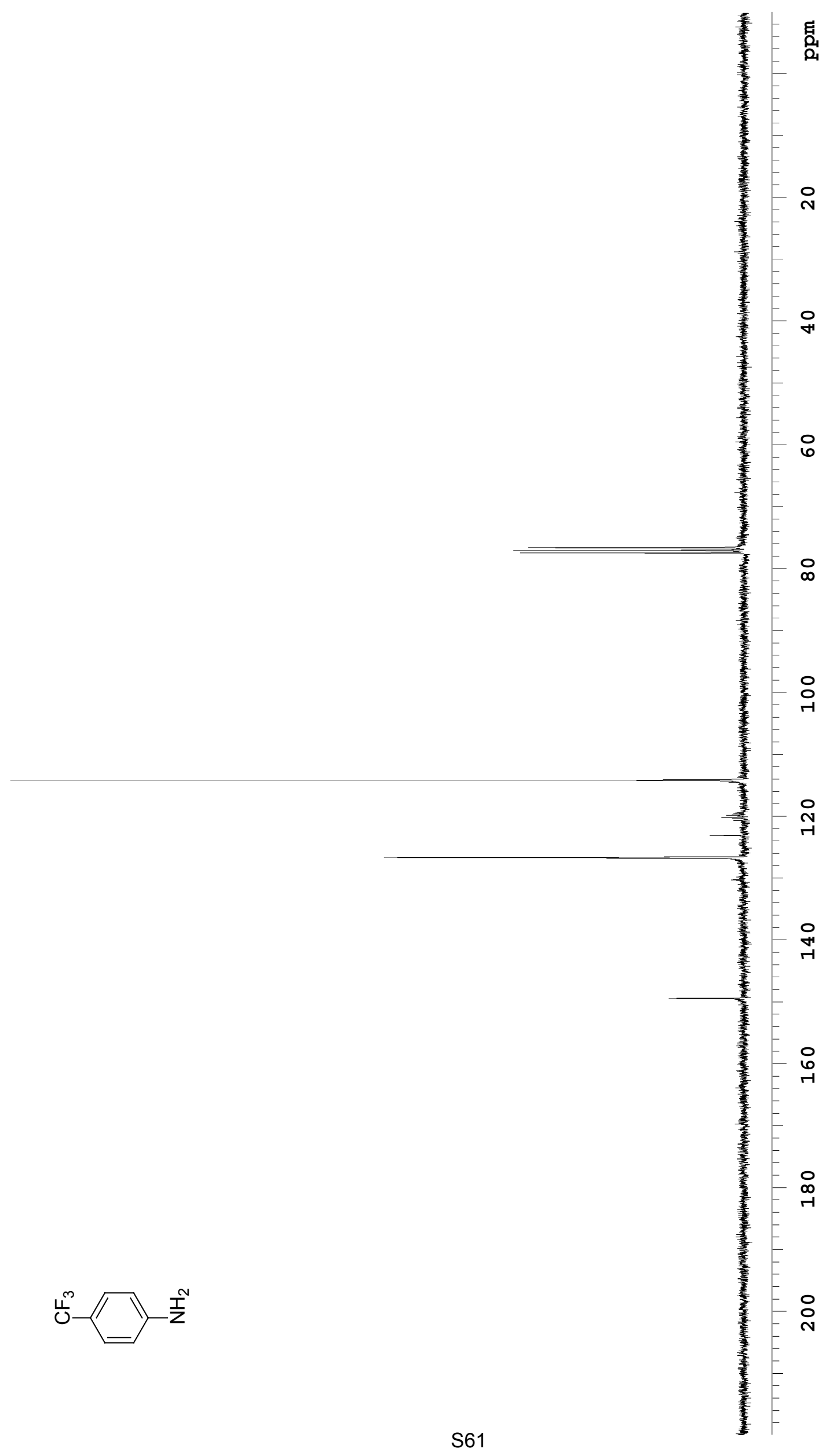




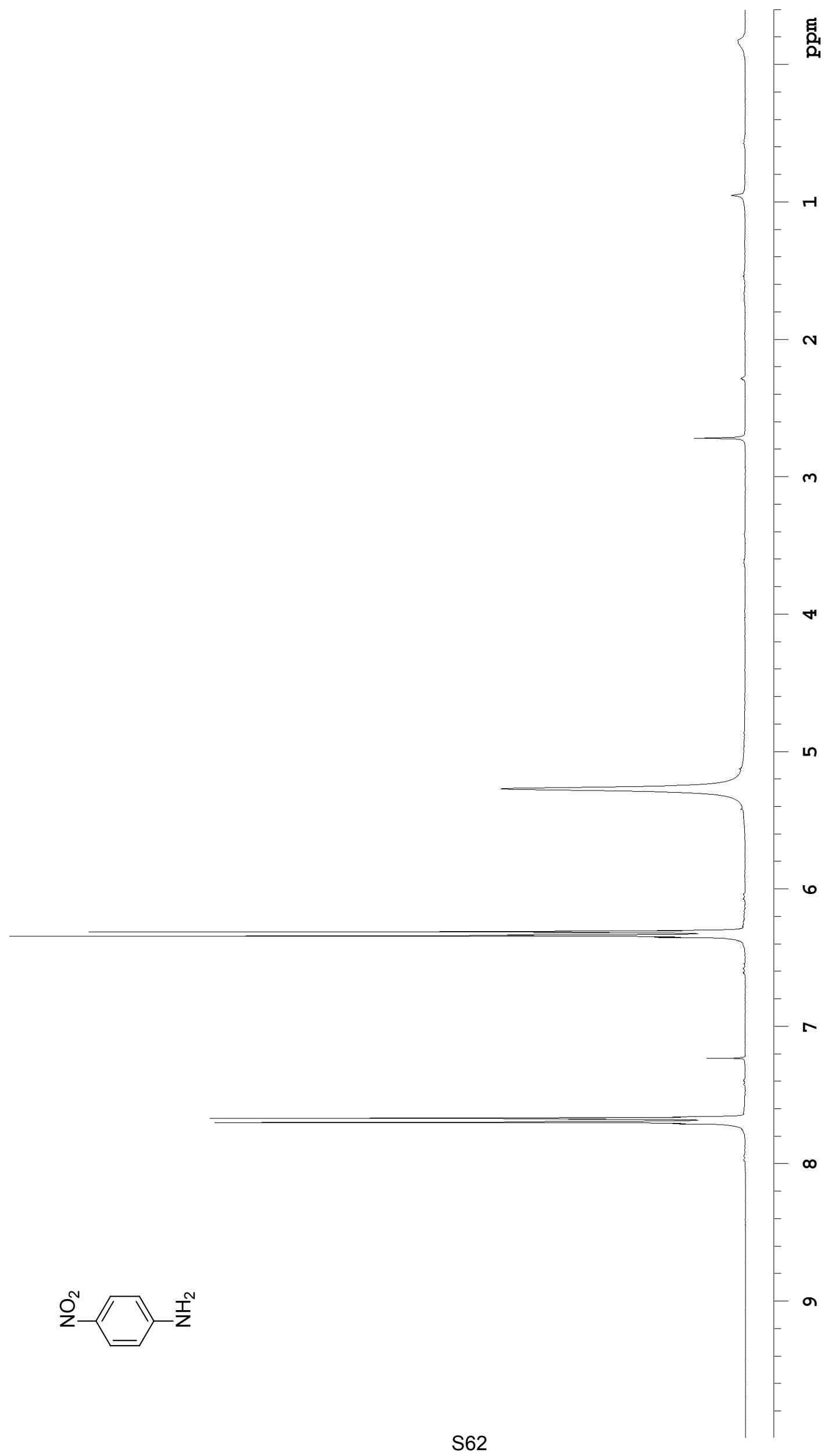




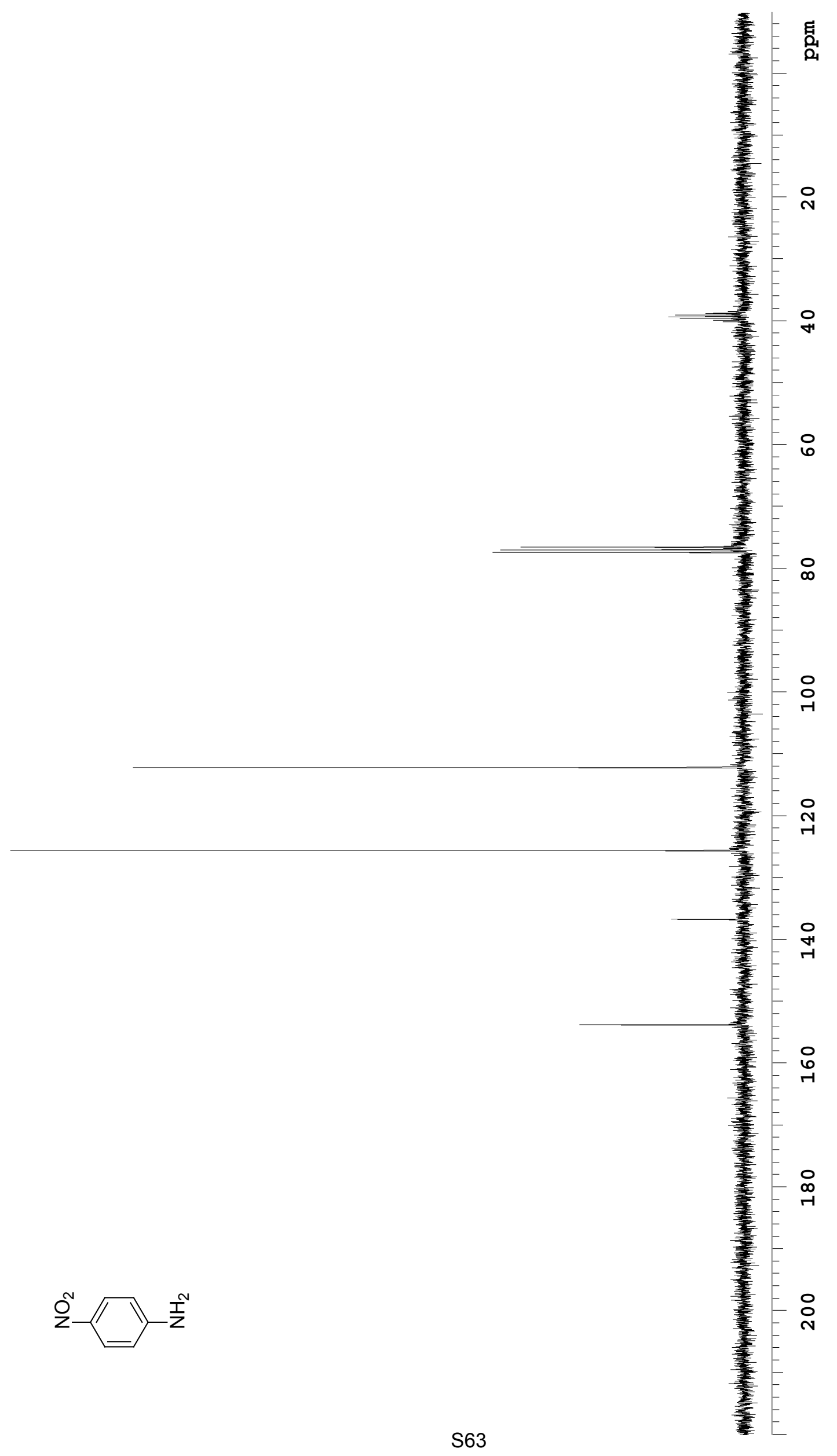




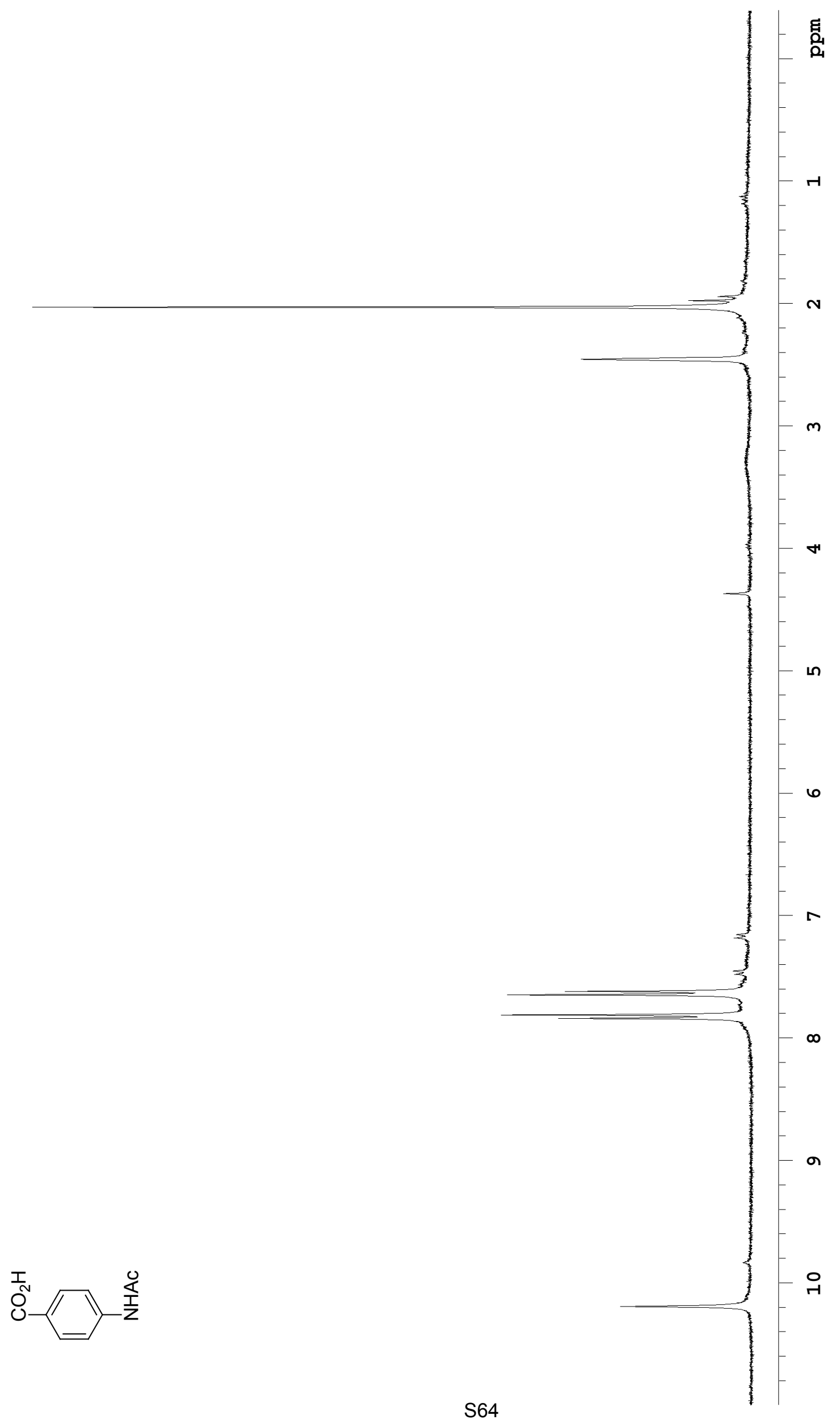




$$
1
$$




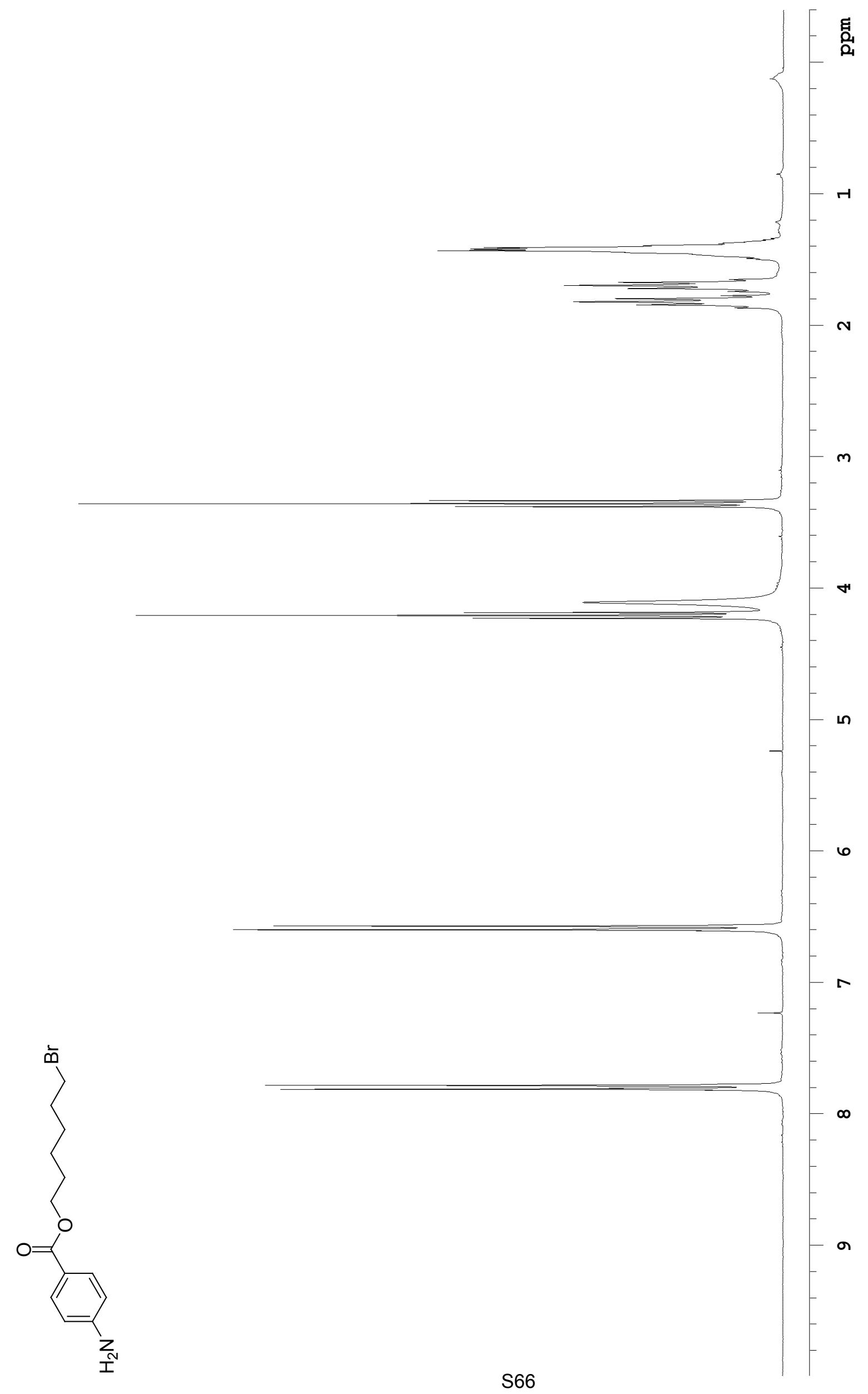




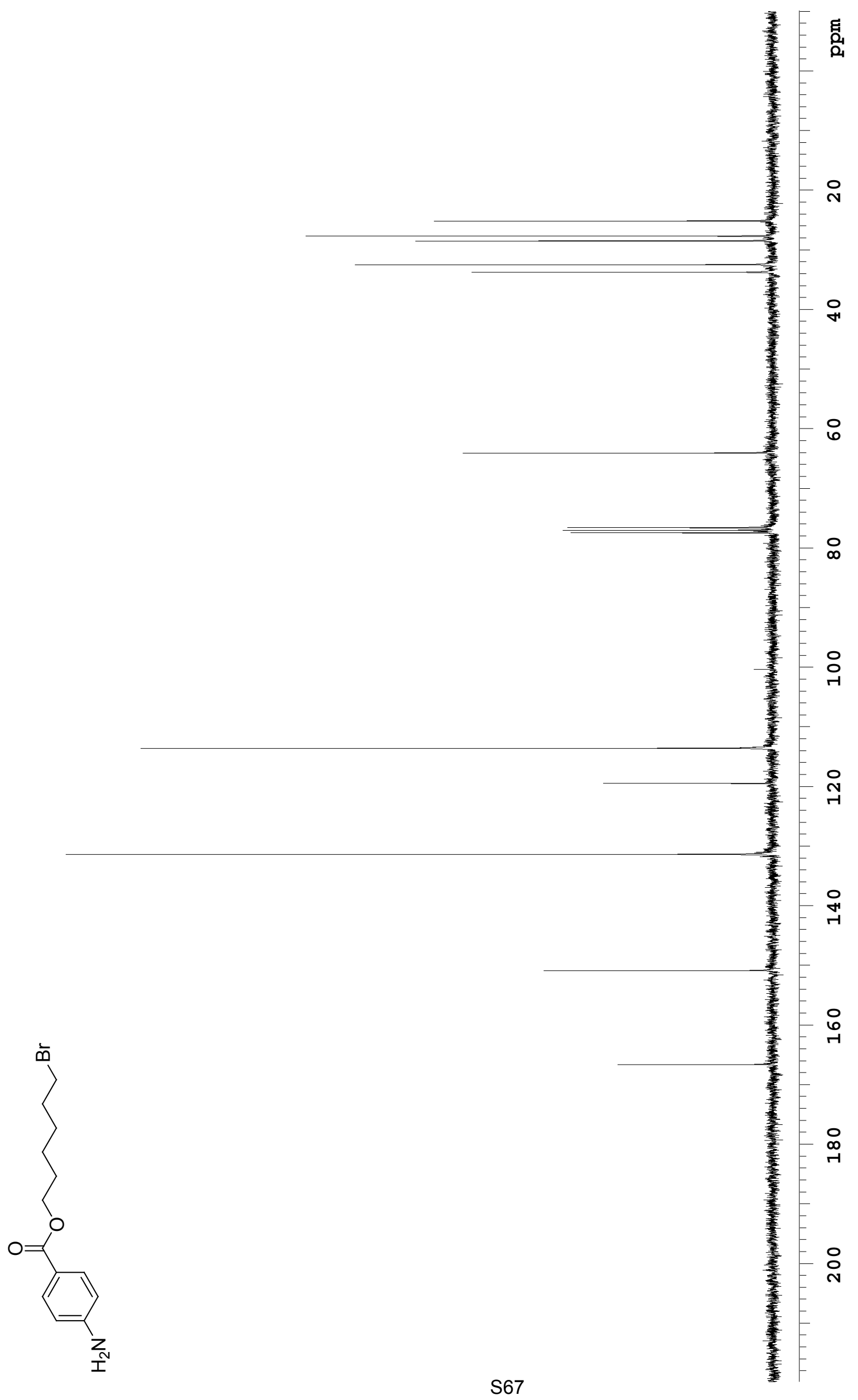




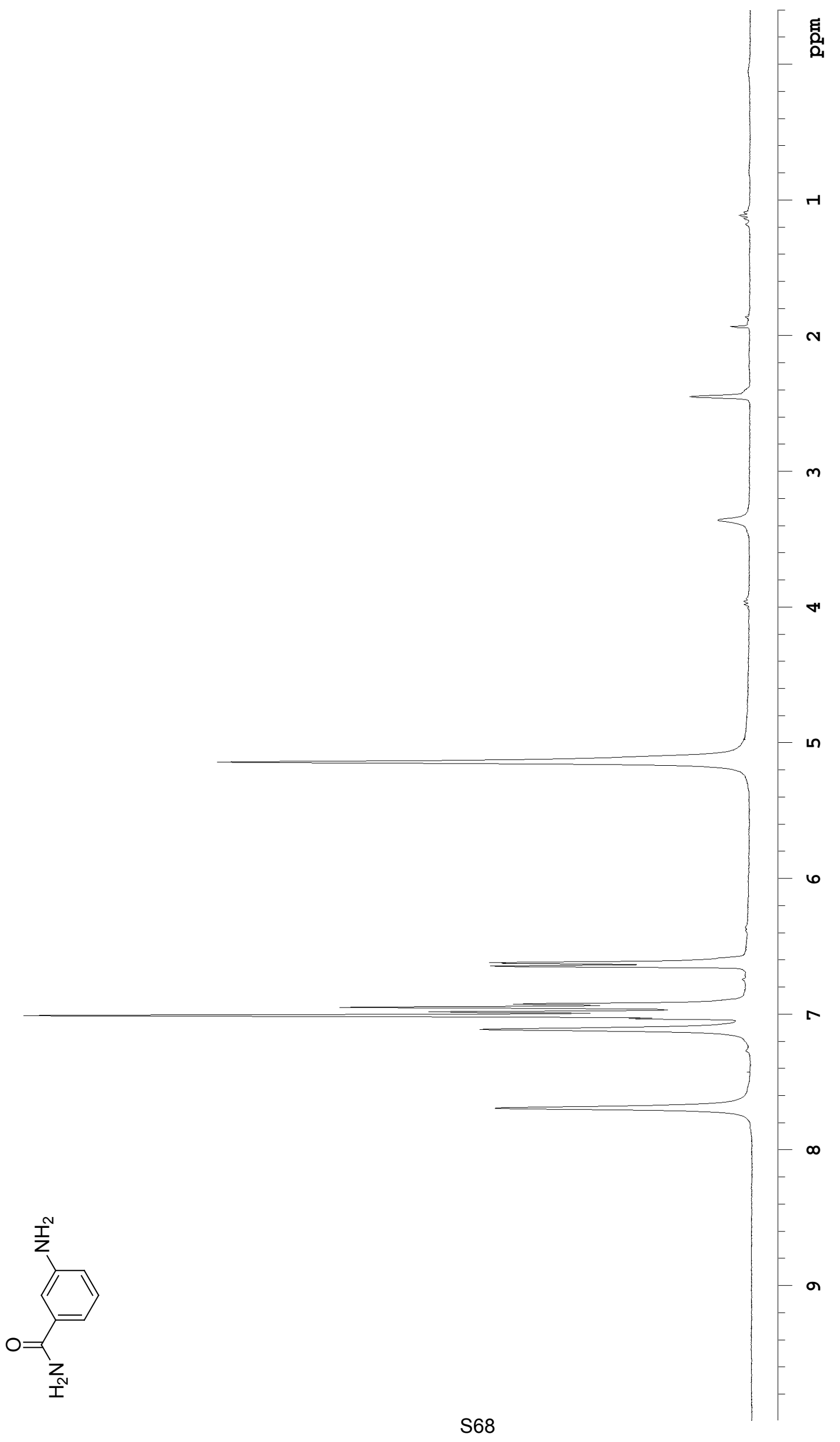




$$
1
$$




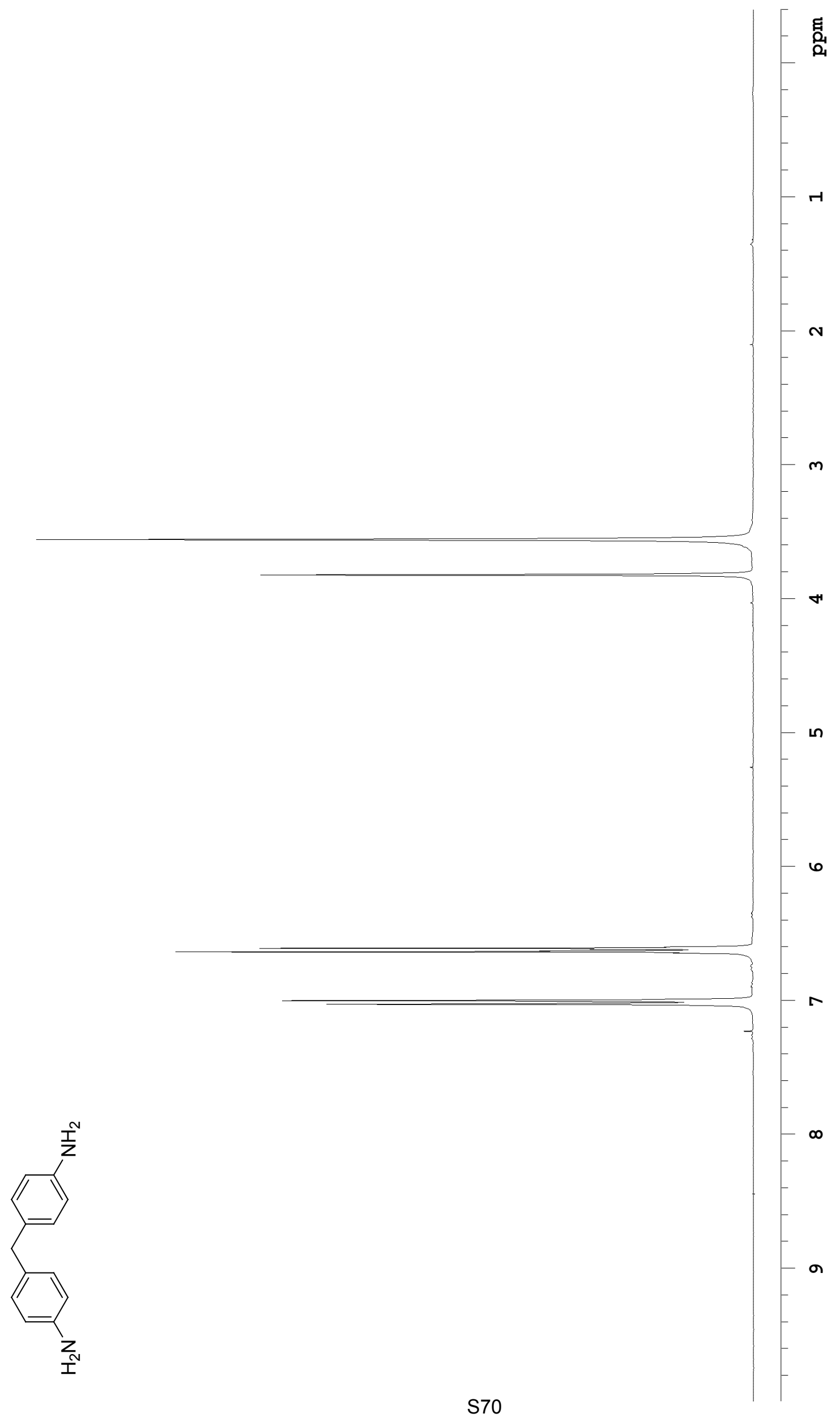




$$
1
$$




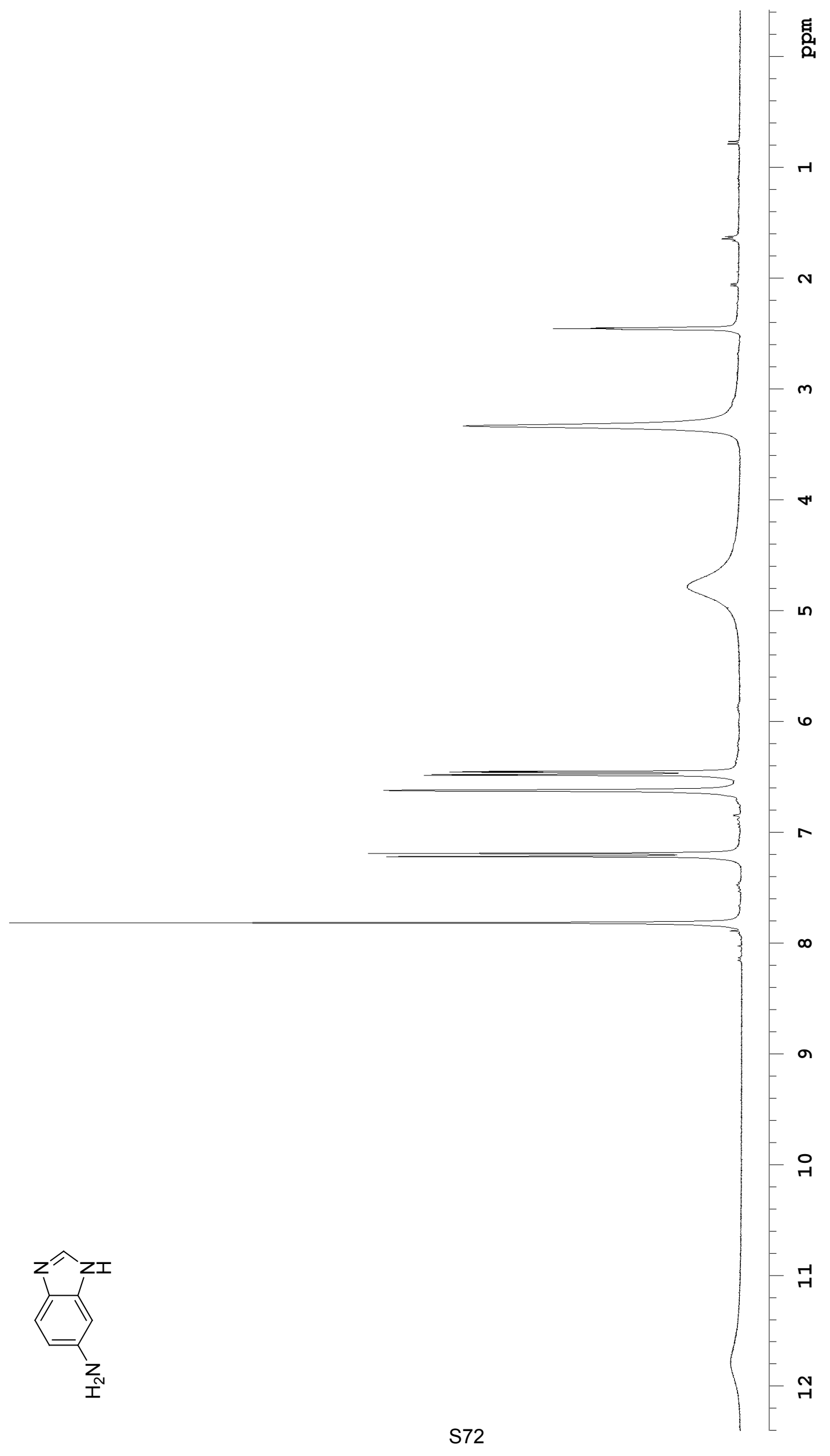




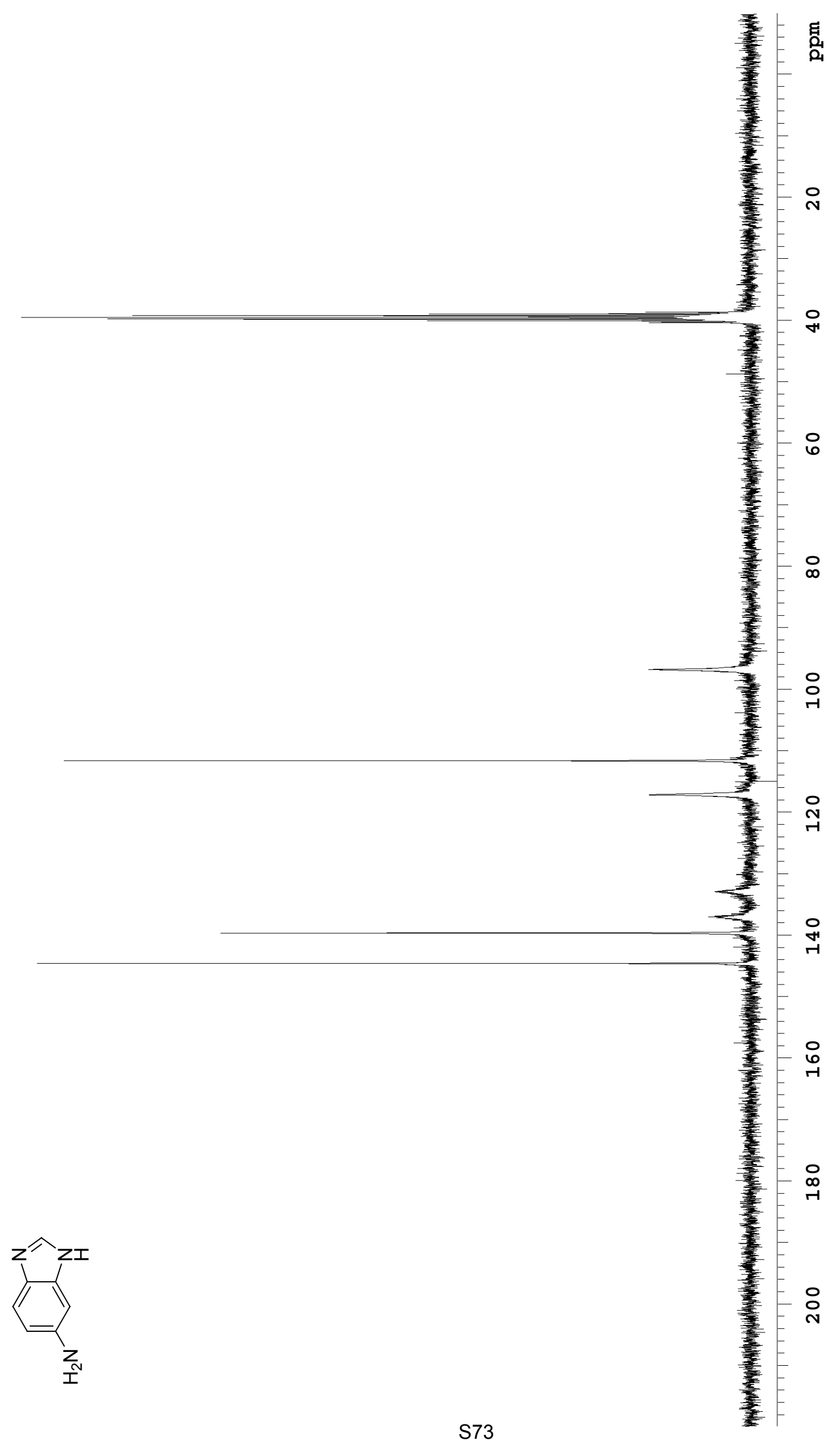




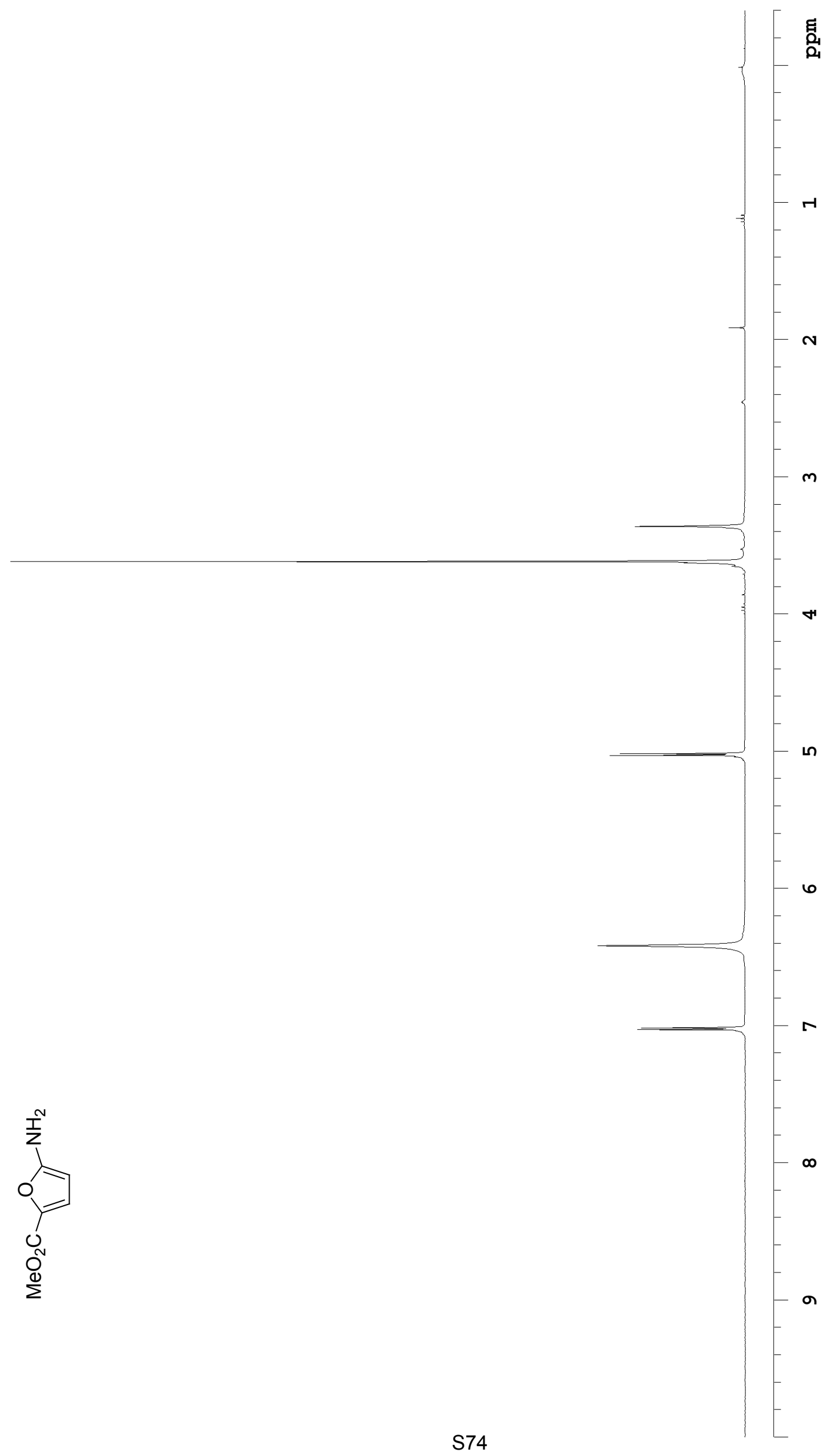




$$
1
$$




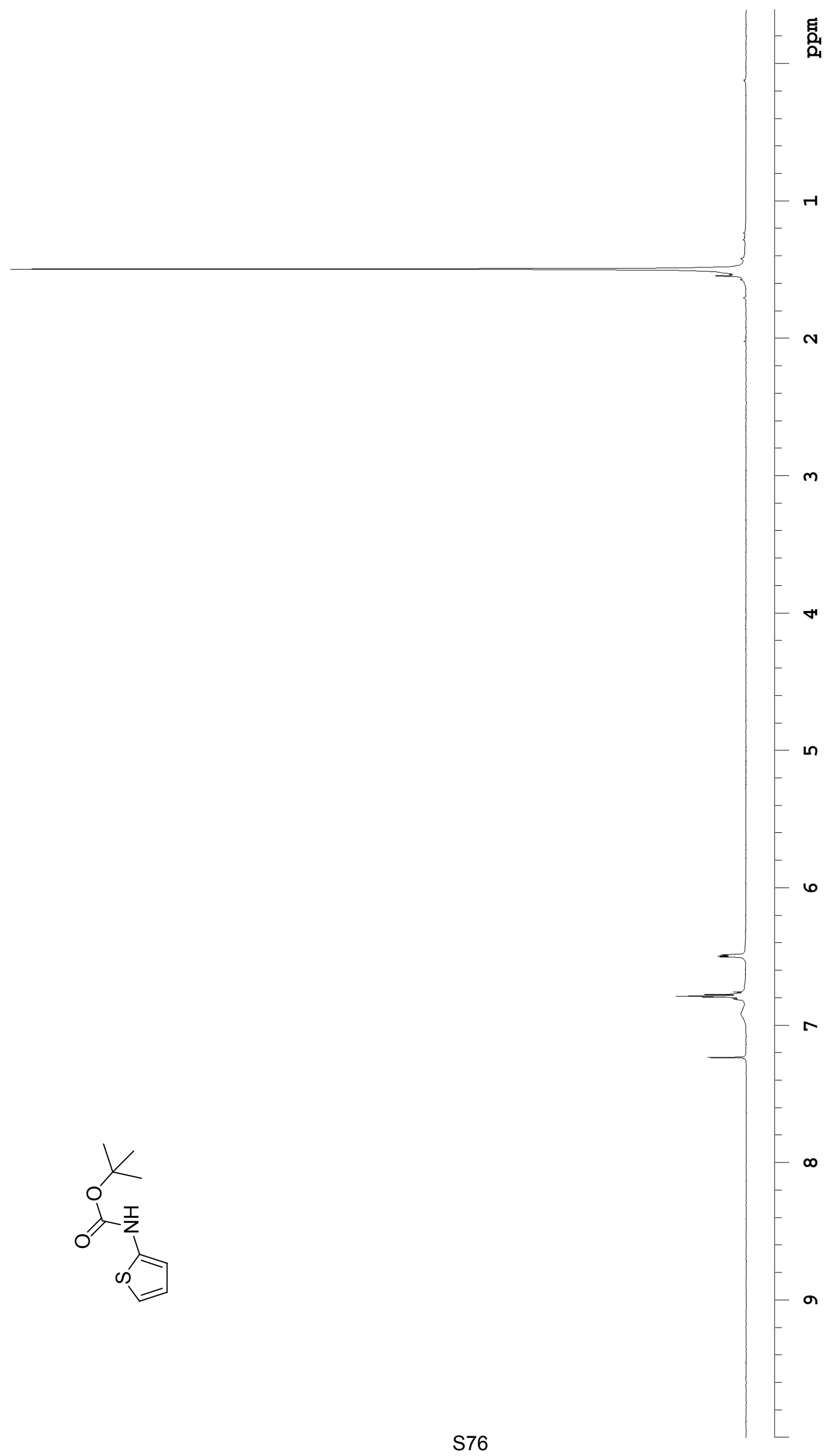




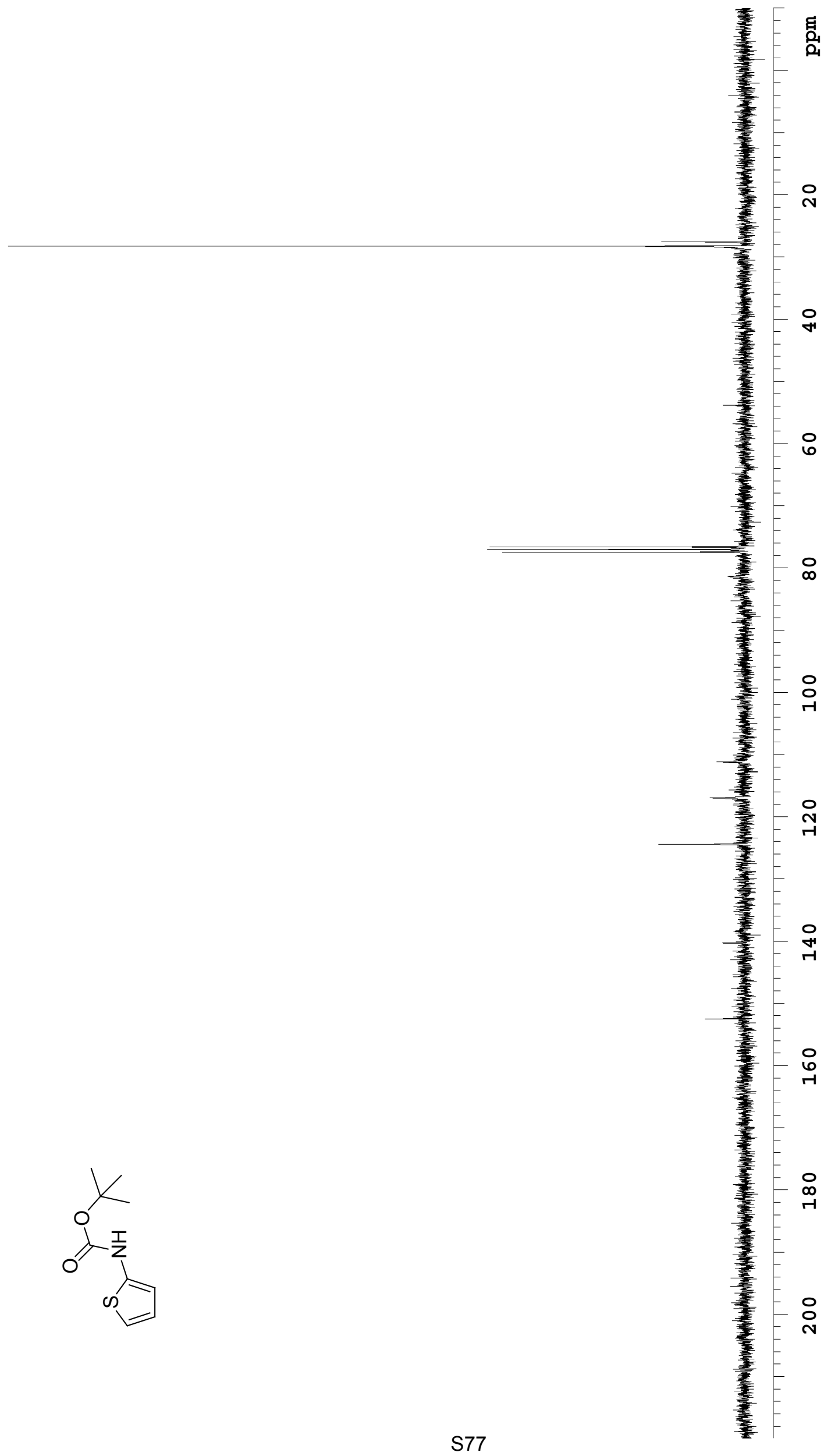




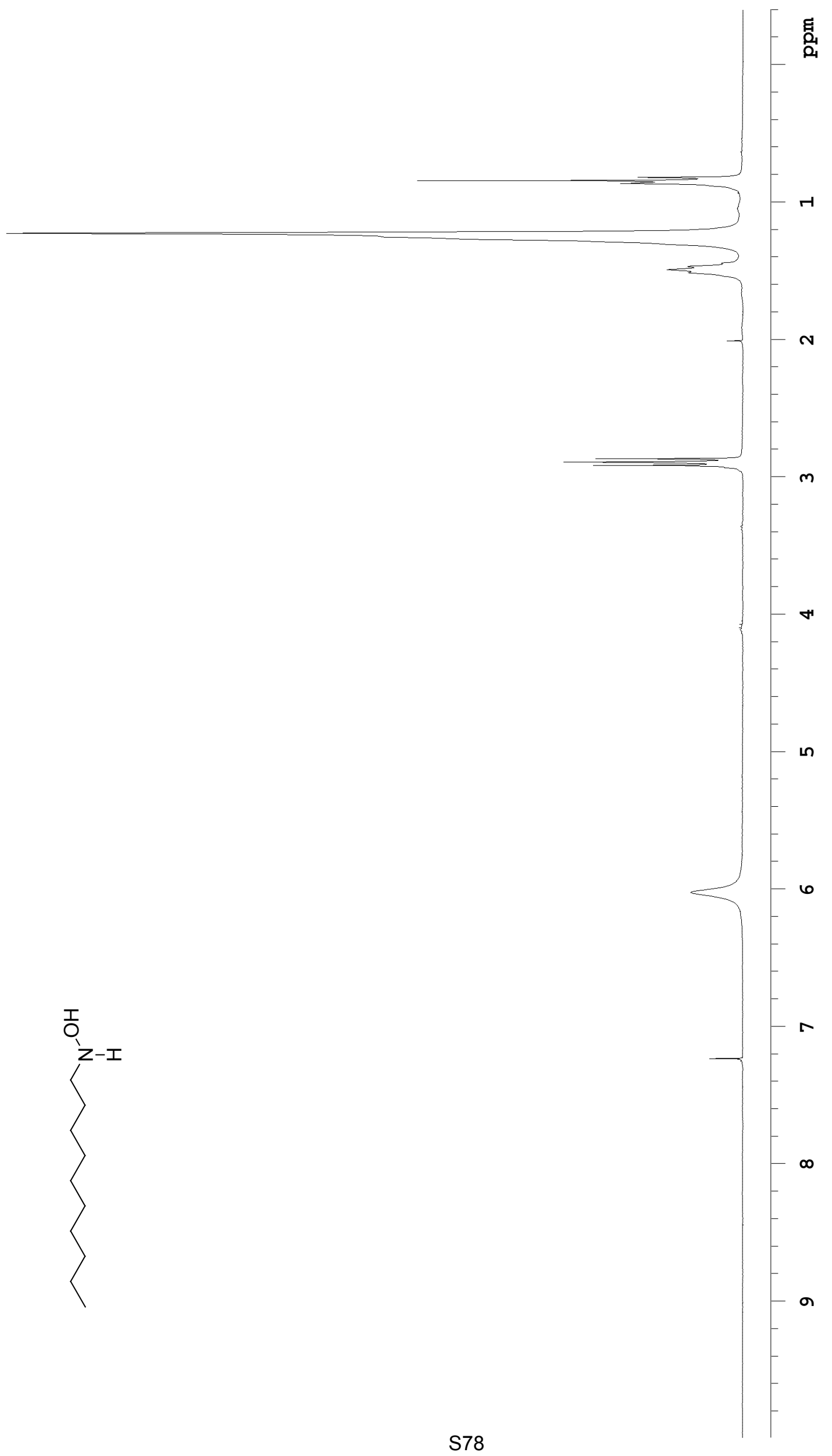




$$
1
$$




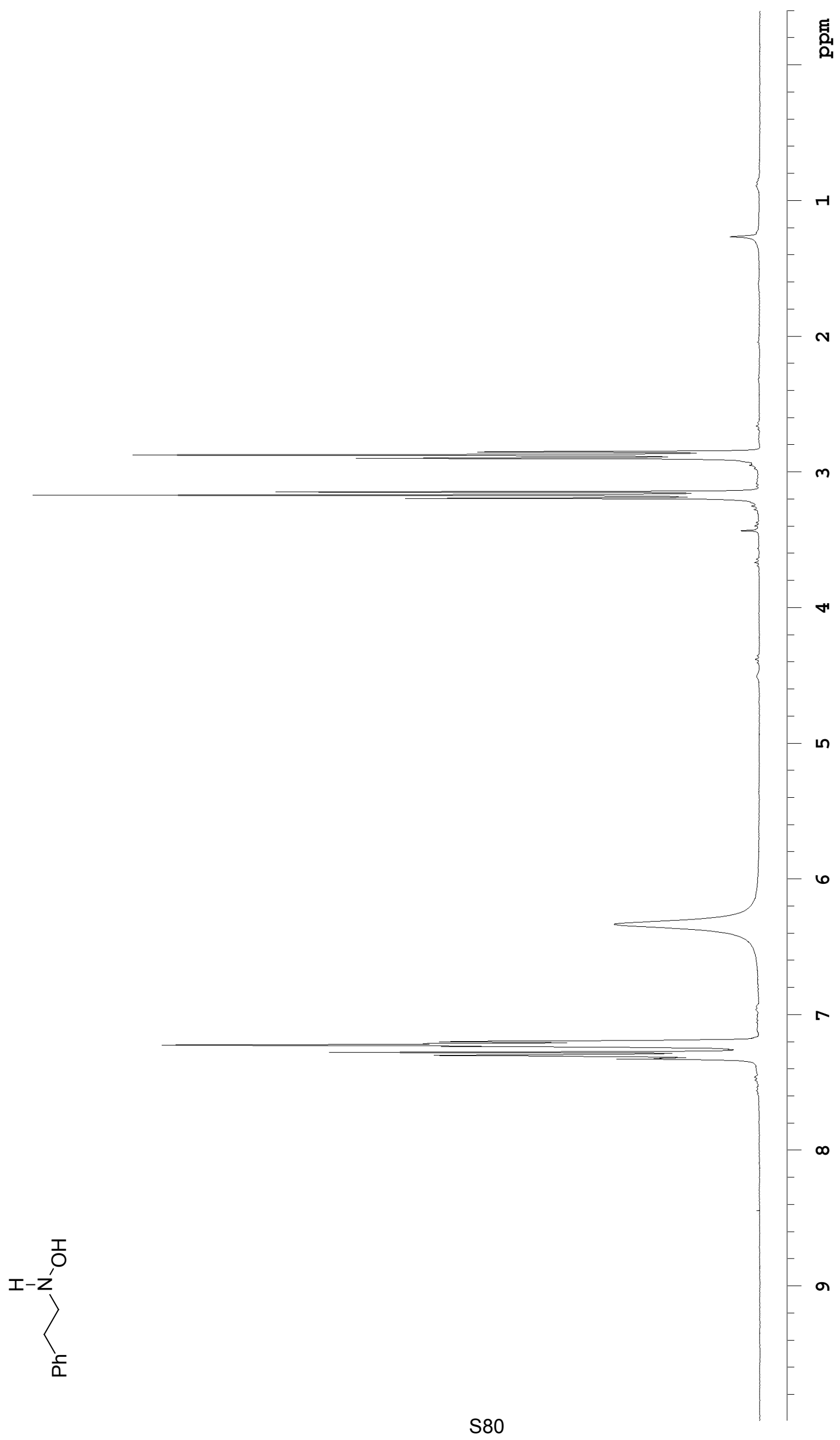




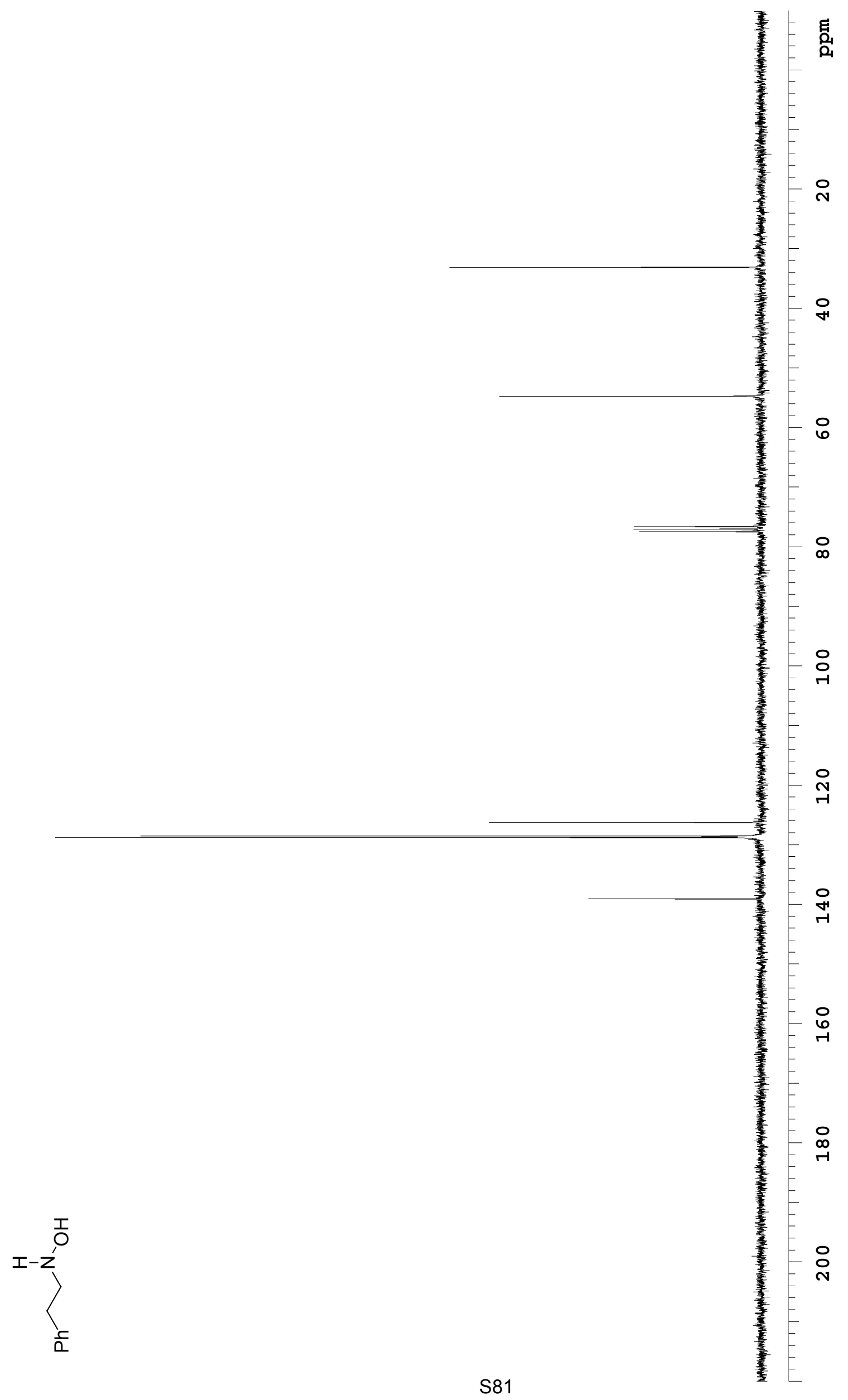




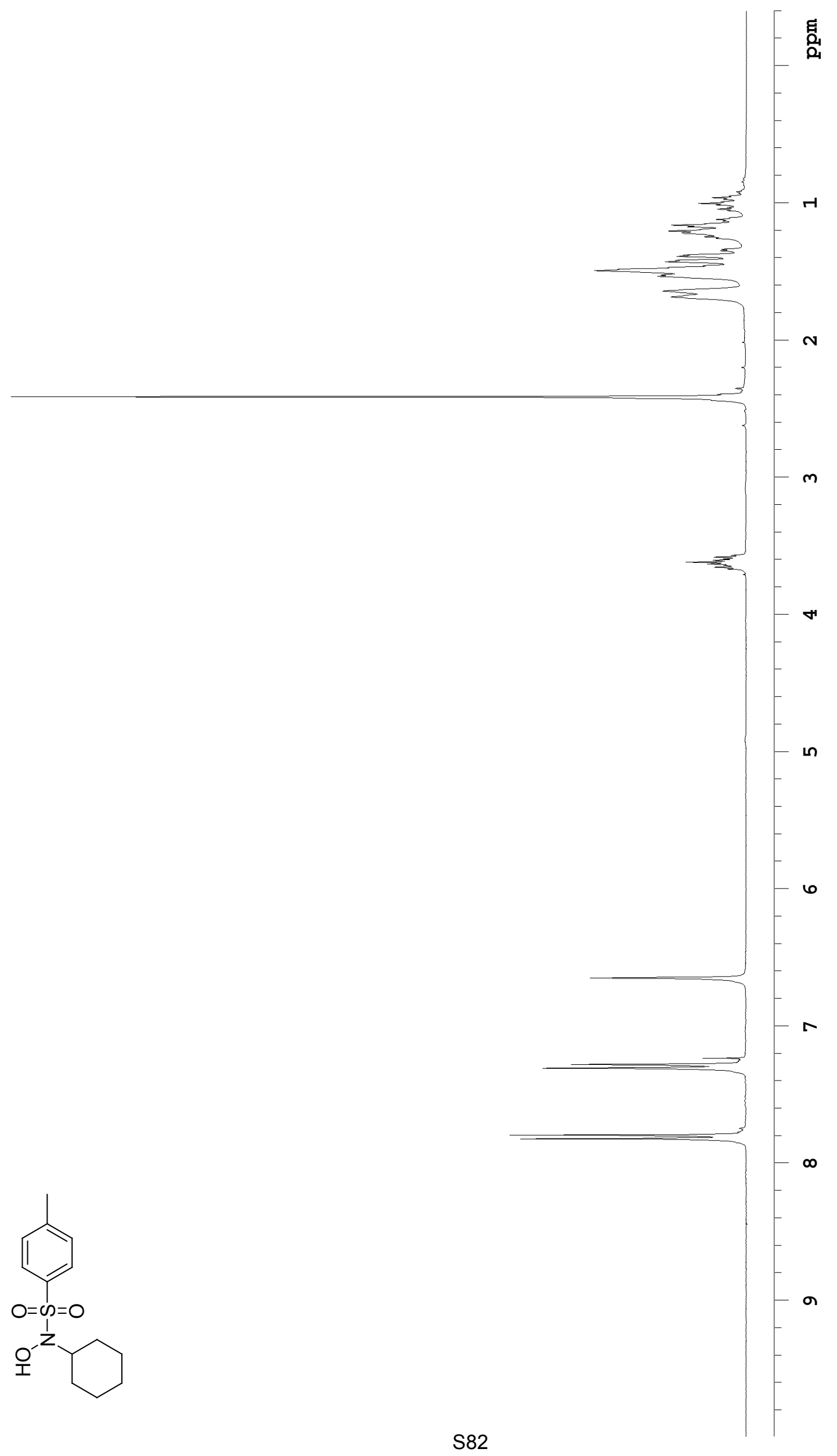




$$
1
$$




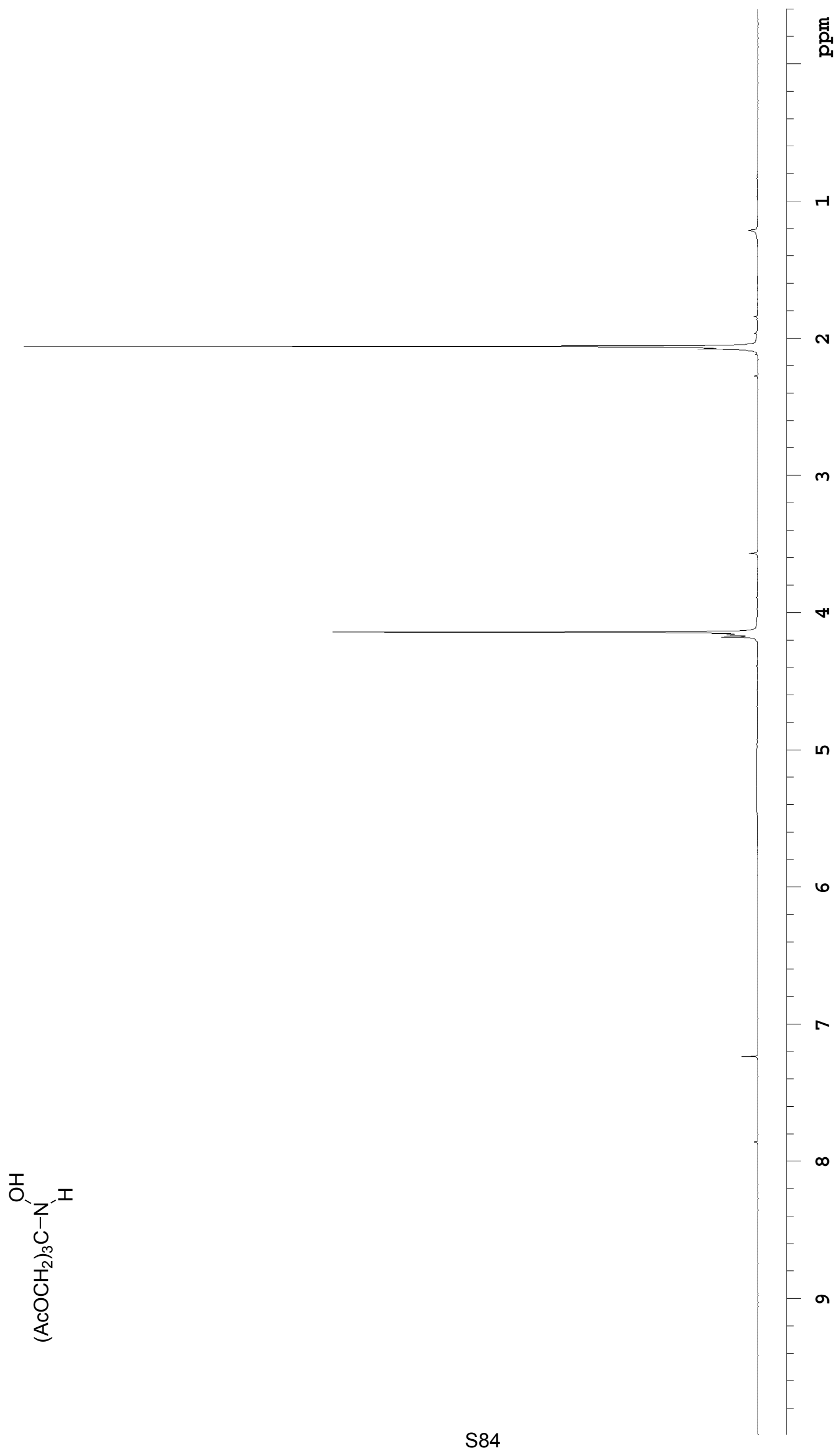




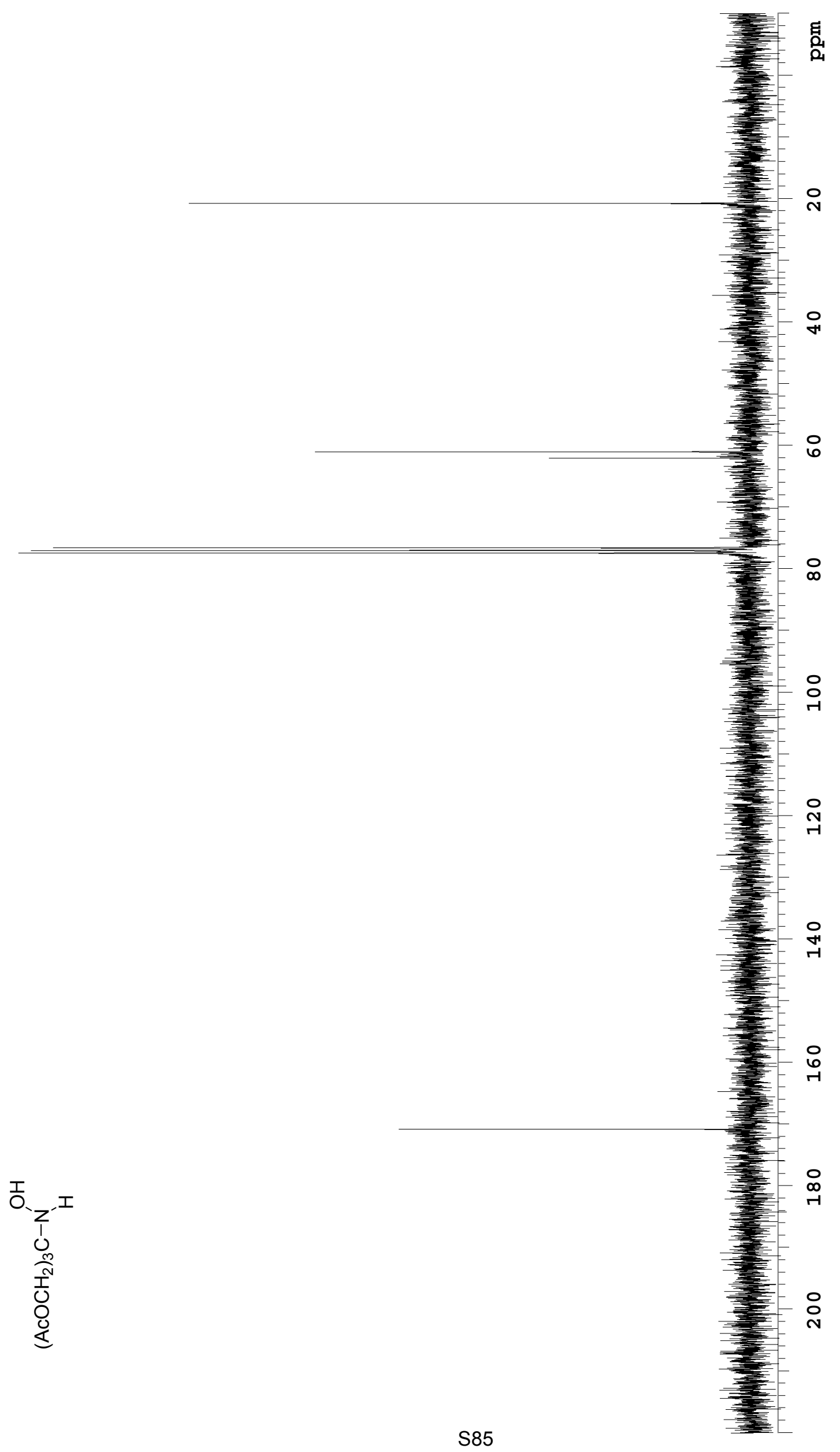




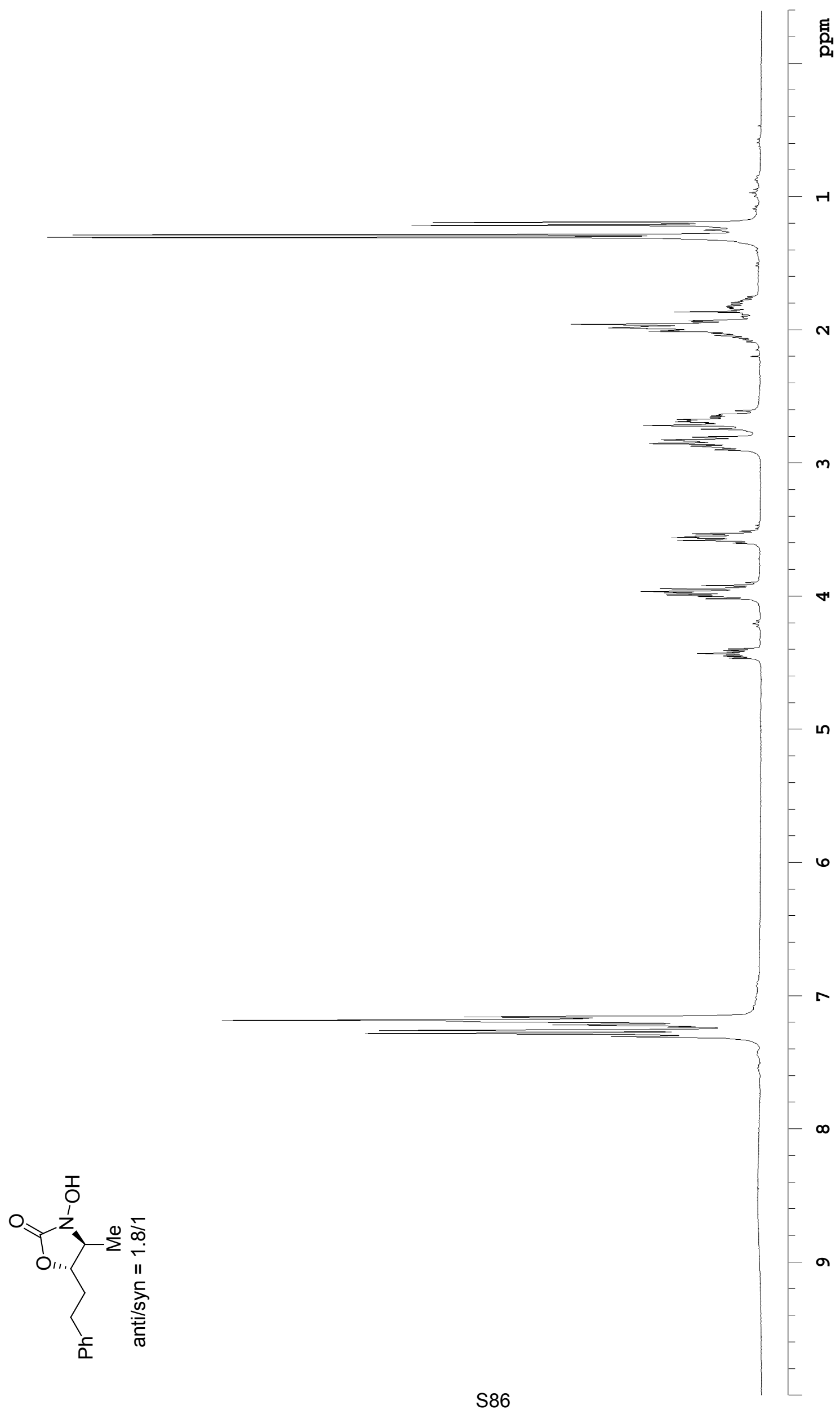




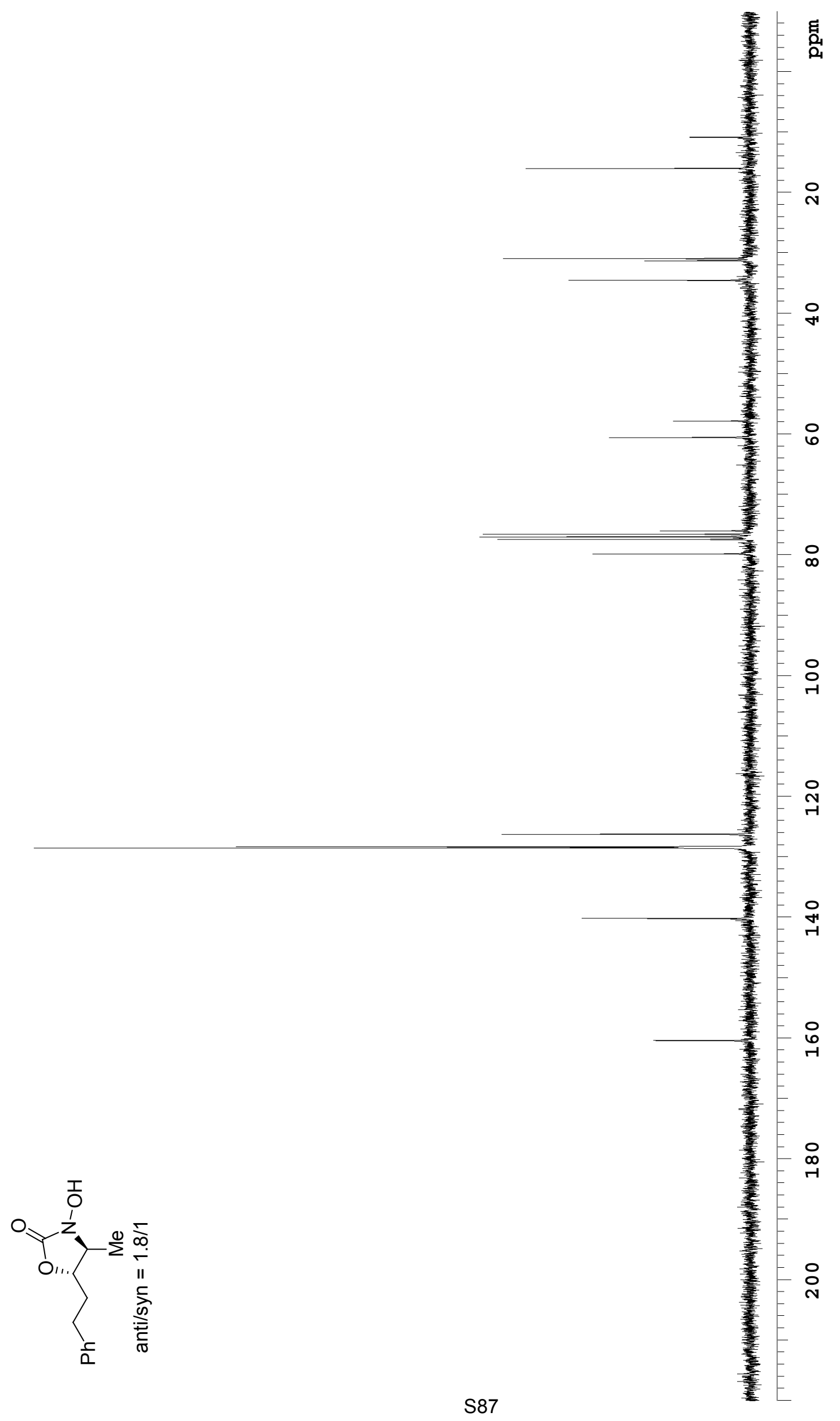




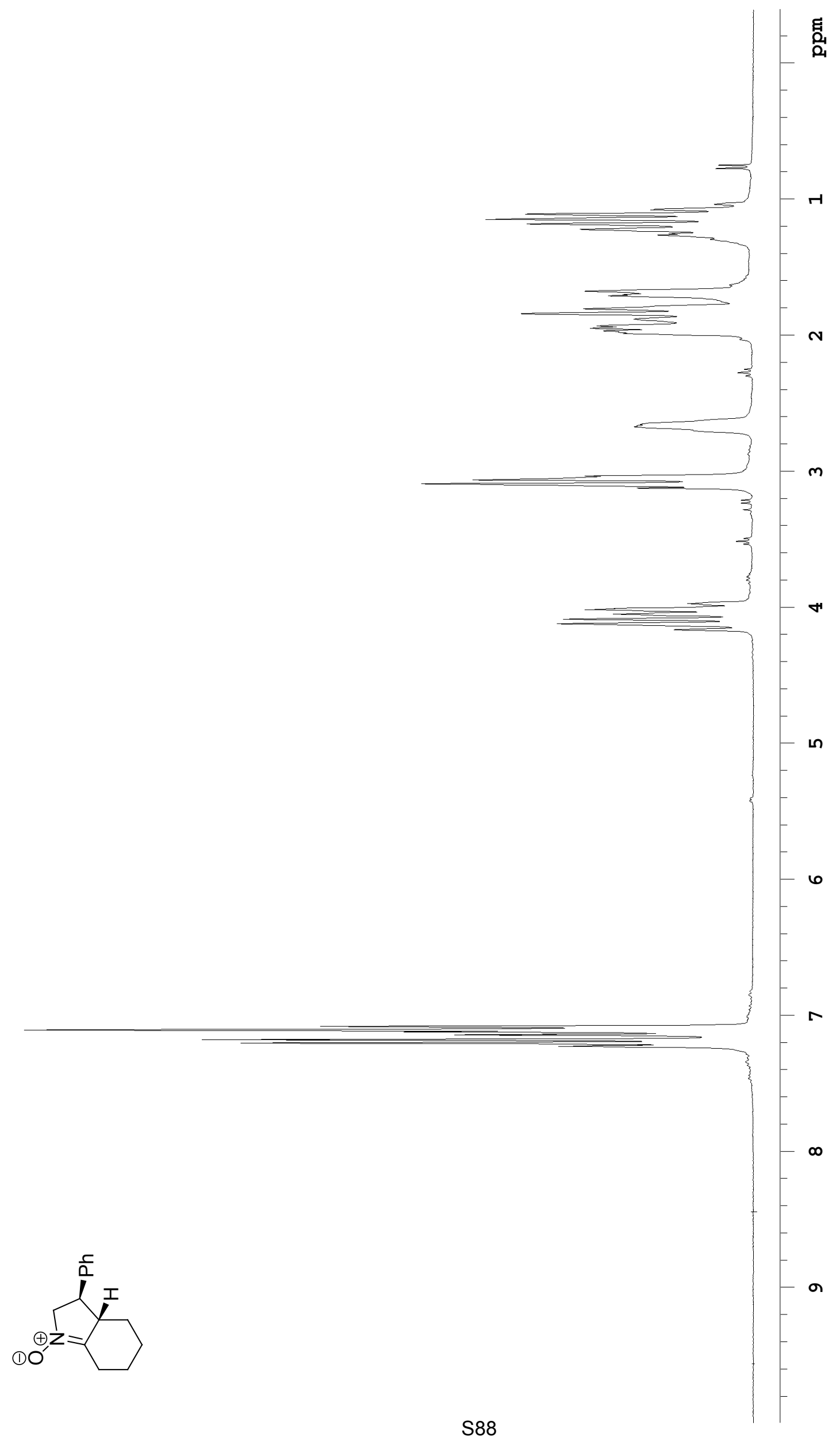




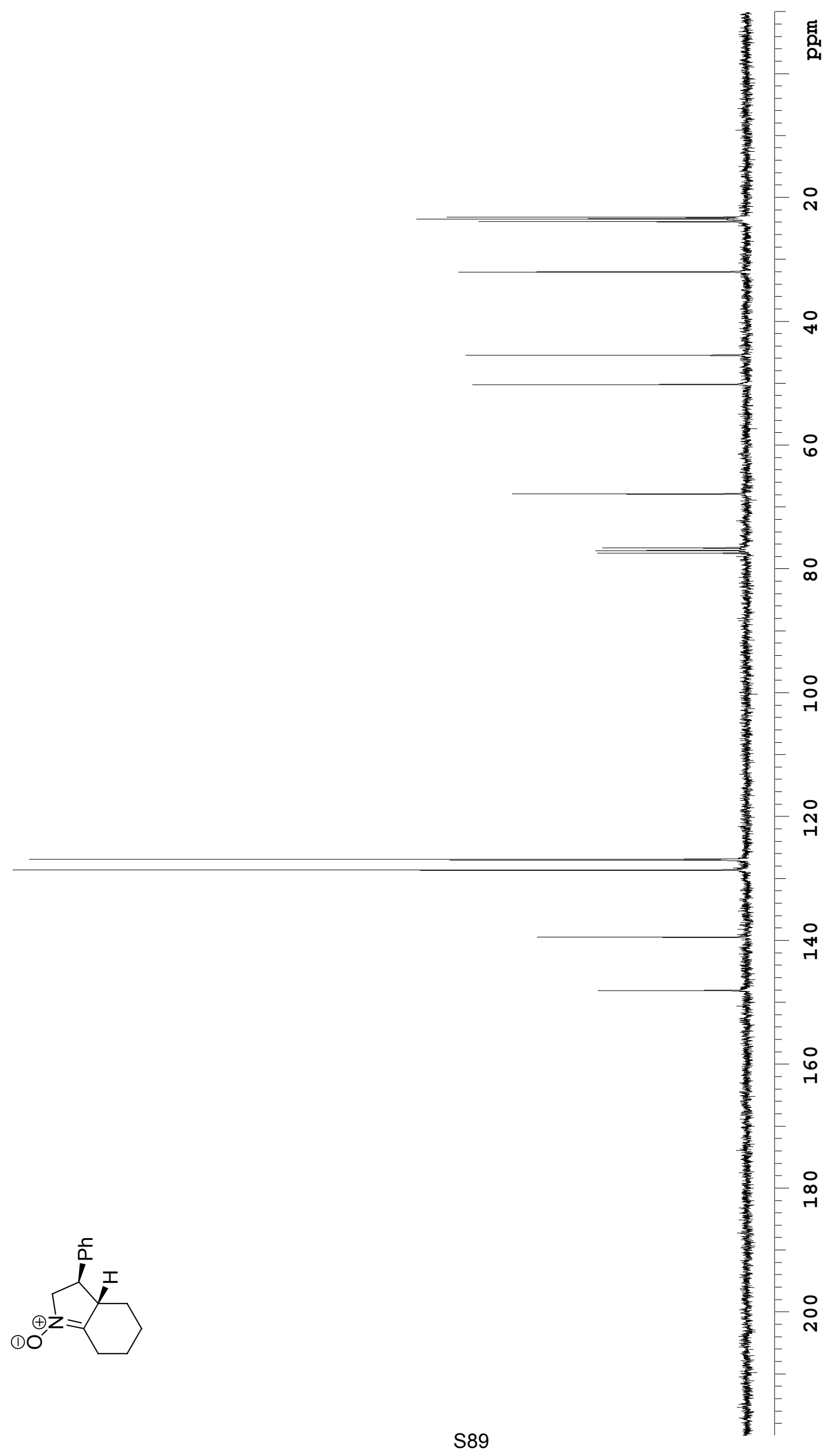

